\title{
Anisotropic potential of velocity fields in real fluids: Application to the MAST solution of shallow water equations
}

\author{
Costanza Aricò*, Marco Sinagra, Tullio Tucciarelli \\ Dipartimento di Ingegneria Civile, Ambientale, Aerospaziale, dei Materiali (DICAM), Università di Palermo, Viale delle Scienze, 90128 Palermo, Italy
}

\section{A R T I C L E I N F O}

\section{Article history:}

Received 16 May 2013

Received in revised form 8 August 2013

Accepted 19 September 2013

Available online 27 September 2013

\section{Keywords:}

Shallow waters

Potential flow problem

Unstructured mesh

Delaunay triangulation

Numerical methods

Dam-break

\begin{abstract}
A B S T R A C T
In the present paper it is first shown that, due to their structure, the general governing equations of uncompressible real fluids can be regarded as an "anisotropic" potential flow problem and closed streamlines cannot occur at any time. For a discretized velocity field, a fast iterative procedure is proposed to order the computational elements at the beginning of each time level, allowing a sequential solution element by element of the advection problem. Some closed circuits could appear due to the discretization error and the elements involved in these circuits could not be ordered. We prove in the paper that the total flux of these not ordered elements goes to zero by refining the computational mesh and that it is possible to order all the remaining elements by neglecting the minimum inter-element flux inside each circuit, with a very small resulting error.

The methodology is then applied to the solution of the 2D shallow water equations. The governing Partial Differential Equations are discretized over a generally unstructured triangular mesh, which attains the generalised Delaunay property. Solution is obtained applying a prediction-correction time step procedure. The prediction problem is solved applying a MArching in Space and Time (MAST) procedure, where the computational elements are required to be ordered and explicitly solved. In the correction step, a large linear well-conditioned system is solved. Model results are compared with experimental data and other numerical literature results. Computational costs have been estimated and the convergence order has been investigated according to a known exact solution.
\end{abstract}

(c) 2013 Elsevier Ltd. All rights reserved.

\section{Introduction}

The 2D Saint-Venant (SV) [39], or shallow water equations (SWEs), are extensively used for hydrodynamic simulations in rivers, lakes, estuaries and floodplains.

Among all the simplified forms of SWE, the diffusive model has shown robustness with respect to the input data approximations and has provided higher order accuracy with respect to the kinematic wave and the uniform formulae (see [8] and cited references). There are several reasons to prefer the diffusive form to the fully dynamic one. The most important is that the sensitivity of the computed water depth to the topographic error is much higher in the fully dynamic model than in the diffusive one [8]. However, when inertial terms play a major role in hydrodynamic simulations (e.g. sudden failure of a dam or a dyke, transport problems dominated by short period waves), it is necessary to solve the original SWEs, in order to get a good representation of the physical process.

Several numerical models based on the Finite Difference (FD), Finite Volume (FV) and Finite Element (FE) discretization of the

\footnotetext{
* Corresponding author. Tel.: +39 09123896573.

E-mail addresses: costanza.arico@unipa.it, arico@idra.unipa.it (C. Aricò) marco.sinagra@unipa.it (M. Sinagra), tullio.tucciarelli@unipa.it (T. Tucciarelli).
}

SWEs over structured/unstructured meshes have been developed in the last two, three decades. Most of the research effort, especially in the case of FV Godunov-type schemes, has been dedicated to improve solution accuracy and stability, because of the imbalance existing between the source terms and the numerical flux terms, mainly in the case of irregular topographies. Many of the proposed approaches provide poor results in stationary or quasistationary cases and fractional step approaches can fail (see [7] and cited references).

In the last two decades, one of the main challenges of the Authors who proposed FV Godunov-type schemes has been to construct a numerical scheme preserving steady states at the discrete level. A numerical scheme is regarded as well-balanced [22] or satisfying the $C$-property $[11,46]$, if it preserves steady states at rest. The concept of $C$-property has been extended to the case of uniform 1D flow in rectangular section [46] and to 2D problems, only over structured meshes [25,31]. The surface gradient method (SGM) [50] is a Godunov-type scheme where, instead of water depth variable, water surface levels are used for data reconstruction. The SGM has been used by the same Authors to deal with bed topography with vertical steps (surface gradient method for steps, SGMS) [51]. Both SGM and SGMS produce accurate solution over structured meshes. 
Triangular mesh is generally the simplest and most convenient method for covering a 2D domain. An advantage of using triangular meshes is their ability to fit arbitrary geometries and to increase the number of elements in high-gradient topography regions or in regions of particular interest. Many Authors proposed numerical schemes dealing with triangular meshes, where splitting techniques are proposed for the solution of the homogeneous form of the SWEs and the numerical fluxes [4], or for the inviscid and viscous terms of the SWEs, or for the friction and bed slope components of the source terms [24]. Usually these methods solve a Riemann problem at each element interfaces and result computationally very expensive.

Adaptive shallow flow model based on boundary-fitted curvilinear grids have been also proposed [26], where grid elements can change size according to local flow features without altering the total number of elements. An advantage of such an approach is the accurate description of curved shorelines, even though the highly stretched curvilinear elements created by the adaptation process may adversely affect solution accuracy and stability. Examples of adaptive shallow flow models based on unstructured triangular grids are given in [41,42]. One of the main drawbacks of unstructured grids is the grid connectivity when applied on an adaptive procedure. On the opposite, hierarchical quadtree or tritree grids are created by domain decomposition and their underlying tree structure is easy to interrogate in order to identify neighbouring elements [32].

Several FE approaches have been developed for the SWEs $[28,34,44,52]$, aimed to guarantee stable and non-oscillatory schemes under highly varying flow regimes. FE methods based on the primitive form of the SWEs using discontinuous approximating spaces have also been studied $[2,3,16,17]$. This discontinuous approach (Discontinuous Galerkin, DG) has several appealing features, in particular, the ability to incorporate upwinding and post-processing stability into the solution of highly advective flows. A brief description of the advantages and drawback of FE and DG schemes can be found in [8] and cited references.

Another class of numerical schemes, recently proposed for the solution of hyperbolic problems, are the conservation element and solution element schemes (CE/SE), originally proposed by Chang [15]. These schemes present substantial innovations respect to the more traditional FD, FV or FE schemes, mentioned above. Space and time are treated in a unified way and the governing equations are discretized over a space-time space. More details can be found in [49] and cited references.

Major difficulties in the solution of the SWEs are found over initially dry areas, with moving wetting-drying boundaries. If no special attention is paid, standard numerical procedure may fail near dry/wet front, producing unphysical oscillations and negative water depths. During the last decades, hydrodynamic models have been equipped with Wetting-Drying (WD) algorithms, even though some of them require a significant additional computational cost. See for example in $[20,27,32,33]$ a description of the main categories of WD techniques.

Most of the above-referred methods are limited by the CourantFriedrichs-Levy stability condition.

Since 2007, a different numerical scheme has been proposed for the solution of the 1D and 2D fully dynamic SWEs [6,7]. This is a predictor-corrector scheme, which guarantees local and global mass conservation. The main advantage of this methodology is that, even if the computational effort is almost proportional to the number of computational elements, no evidence of stability restriction on the maximum CFL number has been found. The governing equation system is initially split in a prediction kinematic (or convective) and in a correction diffusive system. The convective problem is solved applying a MArching in Space and Time (MAST) procedure, where the numerical fluxes are computed using an Eulerian approach and the computational elements are required to be ordered and explicitly solved according to a decreasing scalar potential value. The diffusive correction step computes the corrective fluxes by solving a large linear algebraic system obtained after linearization of the problem, with order equal to the elements number and a sparse and symmetric matrix. The discretized formulation of the governing equations allows to handle also wetting and drying processes without any additional specific treatment.

The application of the MAST approach has been previously limited by the use of the scalar potential for the element ordering. This scalar potential does exist only for the solution of the diffusive form of the SWEs, but is missing for the most general velocity field (i. e. fully dynamic SWEs formulation). The element ordering, in the solution of the original fully dynamic SWEs, was achieved by using an approximated potential, which requires the solution of a new algebraic system, as well as an extra correction step [6,7]. In the present paper it is first shown that an "anisotropic" scalar potential always exists for the most general velocity field resulting from the solution of the fully dynamic SWEs, such that its gradient forms always, at any point and at any time, a negative dot product with the velocity vector. Starting from this finding, a procedure is proposed for elements ordering at the beginning of each time level. Due to the discretization error, some closed circuits can appear and the computational elements involved in these circuits could remain not ordered at the end of the procedure, but the corresponding flux goes to zero by refining the computational mesh. A simple procedure is also proposed to cut such circuits and to order anyway all the elements in the domain.

Another significant innovation with respect to the previous algorithm concerns the solution of the diffusive step. Fluxes are discretized according to a formulation similar to the one adopted by the Mixed Hybrid Finite Element (MHFE) schemes [48]. According to a proposed adjustment of the standard MHFE formulation and due to the mesh Delaunay property, the stiffness matrix of the diffusive problem always guarantees the $M$-property, which preserves solution monotonicity [48] (see in Appendix $C$ the basic definition of $M$-matrix).

The paper is organised as follows. In Section 2.1 the $O$ property of a discretized velocity field is defined and its relationship with a possible scalar potential is explained. A discretized velocity field satisfies the $O$ property if it is possible to order all the elements such that the fluxes through the edges of an element with order number $k$ comes either from the boundary or from elements with lower order number (i.e. previously ordered elements). In Section 2.2 the "anisotropic" potential is defined and it is shown to exist for the most general solution of the Reynolds equations. It is also shown that the existence of an "anisotropic" potential guarantees the $O$ property to be asymptotically satisfied with the use of a strong enough mesh density. In Section 2.3 a simple correction to get the $O$ property also with coarse meshes is proposed.

The governing Partial Differential Equations (PDEs) are shown in Section 3. An overview of the proposed computational scheme is given in Section 4, with the proposed innovative details of the prediction and correction problems solution, as well as of the boundary conditions. Finally, several numerical tests are proposed in Section 5, where numerical results are compared with both lab measured data and numerical results computed by other literature schemes. An analysis of the computational costs is also carried out.

\section{The flow field potential and the elements ordering procedure}

\subsection{The isotropic potential and the 0 property}

When an exact scalar potential $P$ of the flow field exists, velocity vector $\mathbf{u}$ has the same direction of the spatial gradient $\overrightarrow{\nabla P}$ of the potential and it is always oriented according to the decreasing potential values, such that: 
$\mathbf{u}=-k_{0} \overrightarrow{\nabla P}$,

where $k_{0}$ is a positive scalar. The velocity fields resulting from the diffusive form of the SW equations or from their stationary case are examples of velocity fields with exact potential, that in the following we shall call also isotropic potential. The exact (isotropic) potentials in these cases are respectively the piezometric head and the hydraulic head.

In the following we shall assume the control volumes obtained after space discretization of the computational domain to overlap with the mesh elements. Most of the available numerical schemes associate to each control volume a single potential value (at the nodes for standard (e. g. Galerkin) FE schemes, or at the circumcentres for FV or MHFE methods) and guarantee the flux between two neighbouring elements to be oriented from the highest to the lowest potential value [6,7]. This implies that it is always possible to order all the elements according to their decreasing potential value, such that the following property (that we shall call $O$ property from now on) is satisfied for the ensemble of the elements: each element has an order number and the fluxes entering in any element with order $k$ come either from the boundary or from elements with lower order. It can be seen in references [6,7] that the existence of such ordered set is a necessary condition for a possible sequential solution of the averaged governing equations in each element.

On the other hand, it would be possible to obtain the same element ordering by applying the following procedure, even if the actual potential value were left unknown at each time level. Let $T_{e}$ be a generic element. We define $n o_{e}^{k}$ the order number of element $T_{e}$ at time level $t^{k}$.

(1) Assign $n o_{e}^{k}=0$ to all elements.

(2) Assign $n o_{e}^{k}=1$ to those elements $T_{e}$ whose sides fulfil the following requirement: internal sides have only zero or outward oriented fluxes.

(3) Iterate the following procedure until at least one new element is ordered in the last iteration, or all the elements are ordered:

(a) select all the elements $T_{e p}$ with $n o_{e p}^{k}=0$ (i. e. not yet ordered element) which satisfy the following conditions: internal sides have either zero fluxes or inward directed fluxes only from neighbouring ordered elements $T_{e m}$ with $n o_{e m}^{k}>0$ (i. e. already ordered element) and boundary sides have either assigned inward or outward oriented fluxes.

(b) assign to each selected element order number $n o_{e p}^{k}=m+1$, where $m$ is the maximum order of its neighbouring elements.

We can show now that, if a scalar (even unknown) potential is associated to each element and all the internal fluxes are oriented from the higher to the lower potentials, the set of the remaining unordered elements (i.e. with $n o_{e}^{k}=0$ ) is empty. To this end observe that, if $n o_{e p}^{k}=0$, at least one of its internal sides must have an inward oriented flux (otherwise element $T_{e p}$ would satisfy the requirement of step (2) and $n o_{e p}^{k}=1$ ). Among all the neighbouring elements sharing fluxes oriented toward $T_{e p}$, at least one element $T_{e m}$ will have order number $n o_{e m}^{k}=0$. Otherwise, an order number greater than zero would have been assigned to $T_{e p}$ in step (3). The same observation can be repeated for the $T_{e m}$ element and this allows the generation of a subset of elements with order number zero. Since the total number of elements is finite, the generation can continue indefinitely only if some or all the elements of the subset form a closed circuit. Since we have assumed that fluxes move from the higher to the lower potential, a looped subset of connected elements cannot exist and the subset is empty.

\subsection{The anisotropic potential flow field}

We will show in the following that, even if the velocity field is obtained as the numerical solution of the complete SW problem and the mesh elements do not satisfy the $O$ property, the same property is asymptotically attained when the size of the elements goes to zero. This conclusion is based on the existence of an 'anisotropic potential', that is a scalar function of space and time such that:

$\mathbf{u}=-\mathbf{K} \cdot \overrightarrow{\nabla P}$

where $\mathbf{K}$ is a real symmetric positive definite matrix (also function of space and time), that we call in the following "anisotropy matrix". To show the existence of this function $P$, let's start from the general formulation of the Reynolds equations $[30,45]$ :

$\frac{\partial \mathbf{u}}{\partial t}+\mathbf{u} \nabla \mathbf{u}-v \nabla^{2} \mathbf{u}+\nabla\left(\frac{p}{\rho}\right)+\mathbf{g} \nabla z=0$

where $t$ is time, $\mathbf{u}$ is the mean flow velocity vector, $p$ is the mean pressure value, $\rho$ is the fluid density and fluid is assumed barotropic, $z$ is the ground topographic level, $\mathbf{g}$ is the gravitational acceleration with norm $g$. Boussinesq hypothesis has been adopted for the Reynolds stresses, and $v$ is the sum of the water and eddy viscosity coefficients, assumed constant in space and time without loss of generality. Call $s=s(t)$ the abscissa of a generic streamline. Multiplying Eq. (3) by vector $\hat{\mathbf{s}}$, the unit vector tangent to the streamline, and dividing by $g$, one gets:

$\frac{\partial}{\partial s}\left(z+\frac{p}{\gamma}+\frac{u^{2}}{2 g}\right)+\frac{1}{g} \frac{\partial u}{\partial t}=-J$,

where $u=\mathbf{u} \cdot \hat{\mathbf{s}}, J$ is the projection of vector $-v \nabla^{2} \mathbf{u} / g$ along $\hat{\mathbf{s}}$ direction and $\gamma$ is the specific fluid weight $(\gamma=\rho g)$. Assume $u$ to be a smooth enough continuum function in both space and time and define $U$ as:

$U(s, t)=\int_{0}^{s(t)} u(s, t) d s$.

$U$ is a continuous function and $u$ can be written as:

$u=\frac{\partial U(s, t)}{\partial s}=\frac{\partial}{\partial s} \int_{0}^{s(t)} u(s, t) d s$

According to Eqs. (5) and (6) one gets:

$\frac{\partial u}{\partial t}=\frac{\partial}{\partial t}\left(\frac{\partial U(s, t)}{\partial s}\right)=\frac{\partial}{\partial s}\left(\frac{\partial U(s, t)}{\partial t}\right)$

where Eq. (7) is based on the smoothness of $U$ function, which implies the continuity of the second derivatives. From Eqs. (7) and (4) can be written as:

$\frac{\partial P}{\partial s}=-J$,

$P=z+\frac{p}{\gamma}+\frac{u^{2}}{2 g}+\frac{1}{g} \frac{\partial}{\partial t}\left(\int_{0}^{s(t)} u d s\right)$.

Call $P$ anisotropic potential. Observe that in the stationary case (time independent problem), $P$ is equal to the total energy (e.g. the hydraulic head $z+p / \gamma+u^{2} / 2 g$ ) and in the hydrostatic case (velocity is zero), $P$ is equal to the piezometric head $(z+p / \gamma)$. Eq. $(8, a)$ and the positive sign of $J$ imply the following condition:

$-\overrightarrow{\nabla P} \cdot \mathbf{u} \geqslant 0$

Eq. (9) implies that the transformation represented by Eq. (2) is given by a $\mathbf{K}$ positive definite full rate $(3 \times 3)$ tensor. 
Assume at a given time $t^{k}$ the exact velocity field $\mathbf{u}$ to be available. The difference between potential $P$ in two points $a$ and $b$ on the same streamline (with co-ordinate vectors $\mathbf{x}_{a}$ and $\mathbf{x}_{b}$ ) is given by:

$P\left(\mathbf{x}_{b}\right)-P\left(\mathbf{x}_{a}\right)=\int_{a}^{b} \frac{\nabla P \cdot \mathbf{u}}{|\mathbf{u}|} d s$.

Eq. (10), coupled with Eq. (2), provides:

$P\left(\mathbf{x}_{b}\right)-P\left(\mathbf{x}_{a}\right)=-\int_{a}^{b} \frac{\left(\mathbf{K}^{-1} \mathbf{u}\right) \cdot \mathbf{u}}{|\mathbf{u}|} d s$.

Observe that the argument of the integral at the r.h.s. of Eq. (11) is always positive, because $\mathbf{K}^{-1}$ is positive definite; this implies that the difference between $P\left(\mathbf{x}_{b}\right)$ and $P\left(\mathbf{x}_{a}\right)$ is always negative, unless the velocity is zero along all the streamline. Assuming the potential continuity, this also implies that a closed streamline cannot occur. The above assumption comes from the hypothesis of continuity and smoothness of velocity $\mathbf{u}$. In Appendix A we prove that closed streamlines cannot occur also in discontinuous velocity fields.

Assume now the velocity and the anisotropy matrix fields to be approximated respectively by a set of vectors and tensors, piecewise constant inside each element. We apply the same element ordering procedure explained in the previous section. Since in this case, unlike in the previous isotropic one, a scalar potential is not associated to each element, such that the side fluxes move from the higher to the lower potentials, the final subset of elements with order number zero could not be empty, the $O$ property could not be satisfied and the elements of the final subset could form one or more closed circuits.

Call $S$ the set of all the sides common to two elements, following each other in the close circuit and $C$ the set of the circumcentres $C$ of all the elements belonging to the same circuit. Call $L$ the closed path given by the straight lines connecting all $C$ points.

Observe that, if the computed $\mathbf{u}$ velocity is a good approximation of the real one, the potential difference between two circumcentres $a$ and $b \in L$ (with coordinates $\mathbf{x}_{a}$ and $\mathbf{x}_{b}$ ), generally not contiguous to each other, can be obtained by integrating the potential gradient component given by Eq. (2) and approximated as:

$P\left(\mathbf{x}_{b}\right)-P\left(\mathbf{x}_{a}\right) \approx-\sum \int_{c_{i}}^{c_{i+1}}\left(\mathbf{K}^{-1} \mathbf{u}\right) \cdot \hat{\mathbf{n}} d n$

where the sum is extended to all the $i$ straight lines connecting each couple of contiguous circumcentres $c_{i}$ and $c_{i+1}$ in $L$ (with co-ordinates $\mathbf{x}_{c_{i}}$ and $\mathbf{x}_{c_{i+1}}$ ) between $a$ and $b$ and $\hat{\mathbf{n}}$ is the unit vector parallel to the straight lines connecting $c_{i}$ and $c_{i+1}$ (see Fig. 1) ( $\hat{\mathbf{n}}$ is

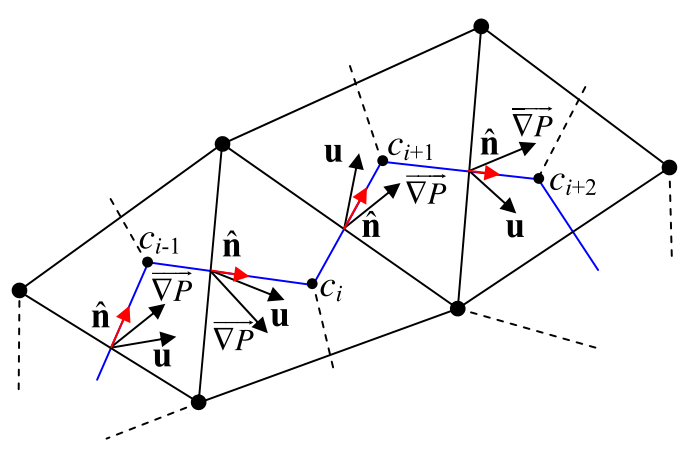

Fig. 1. Computation of potential difference $P\left(\mathbf{x}_{b}\right)-P\left(\mathbf{x}_{a}\right)$ along a closed path. Detail of the approximation of the closed streamline inside some of the involved triangles (blue lines). (For interpretation of the references to colour in this figure legend, the reader is referred to the web version of this article.) orthogonal to the side shared by the two contiguous elements with circumcentres $c_{i}$ and $c_{i+1}$ ). Since $\mathbf{K}^{-1}$ is symmetric, Eq. (12) implies:

$P\left(\mathbf{x}_{b}\right)-P\left(\mathbf{x}_{a}\right) \approx-\sum \int_{c_{i}}^{c_{i+1}}\left(\mathbf{K}^{-1} \hat{\mathbf{n}}\right) \cdot \mathbf{u} d n$.

Moreover, since $\mathbf{K}^{-1}$ is positive definite, the following inequality holds moving according to the flux path orientation:

$\int_{c_{i}}^{c_{i+1}}\left(\mathbf{K}^{-1} \hat{\mathbf{n}}\right) \cdot \mathbf{u} d n \geqslant 0$.

Assuming the continuity of the potential, the velocity and the anisotropy matrix, due to inequality in Eq. (14), the 1.h.s. of Eq. (11) will converge to a negative value along with the increment of the mesh density and a looped subset will finally not exist. The speed of convergence will depend on the actual value of the velocity and of the anisotropy matrix, but we know that, using a dense enough computational mesh, the $O$ property will be finally satisfied. Fig. 2(a) and (b) show a zoom of the computed flow field for one of the following proposed 2D test cases (test 6 in Section 5.6). Both Fig. 2(a) and (b) represent the same portion of the domain. Fig. 2(a) shows the side normal unit vectors, oriented according to the flux sign and obtained by discretizing the domain with a coarse triangulation, while the same vectors in Fig. 2(b) are computed over a refined mesh, obtained from the previous coarse one dividing each element in four equal triangles. Both computed flow fields generate closed circuits (see the blue vectors in the figures), but these reduce by refining the mesh. The mean value of the fluxes along the circuits in the coarse mesh is $8.82329 \mathrm{~d}-04 \mathrm{~m}^{3} / \mathrm{s}$ with a standard deviation 5.3409d-04, while the corresponding values computed for the refined mesh are respectively $4.38 \mathrm{~d}$ $06 \mathrm{~m}^{3} / \mathrm{s}$ and $4.85414 \mathrm{~d}-07$. Refining the computational mesh once again, the closed circuits in the investigated domain area disappear.

\subsection{Flux correction for the achievement of the $O$ property}

To avoid an abnormal increment of the mesh density, it is possible to guarantee the $O$ property by using the original mesh and by setting to zero some of the fluxes through internal sides. To this end, the subset of elements with zero order number is first identified, along with the corresponding loops. The side corresponding to the minimum flux along each loop is then identified and the flux set to zero. The procedure described in Section 2.1 is then started again from step (3). Since the old loops no more exist, one or more elements will be ordered. The new subset with zero order number is computed again and the procedure is repeated until an empty subset is finally found. Numerical values shown in Fig. 2(a) and (b) are the order number of the elements computed after neglecting the minimum flux along the closed path, minus a constant (870). Observe that neglecting a flux through an internal side violates the local mass continuity, but not the global one.

\section{Application to the SWEs}

If the slope of the water surface is small in two horizontal orthogonal directions, velocity and acceleration vertical components in Eq. (3) can be neglected and the vertical distribution of the pressure can be assumed hydrostatic. Averaging the horizontal components of Eq. (3) and the continuity equations along depth, after some manipulations (see for example [1,4,35]) one gets the 2D SWEs [39]:

$\frac{\partial h}{\partial t}+\frac{\partial u h}{\partial x}+\frac{\partial v h}{\partial y}=0$ 


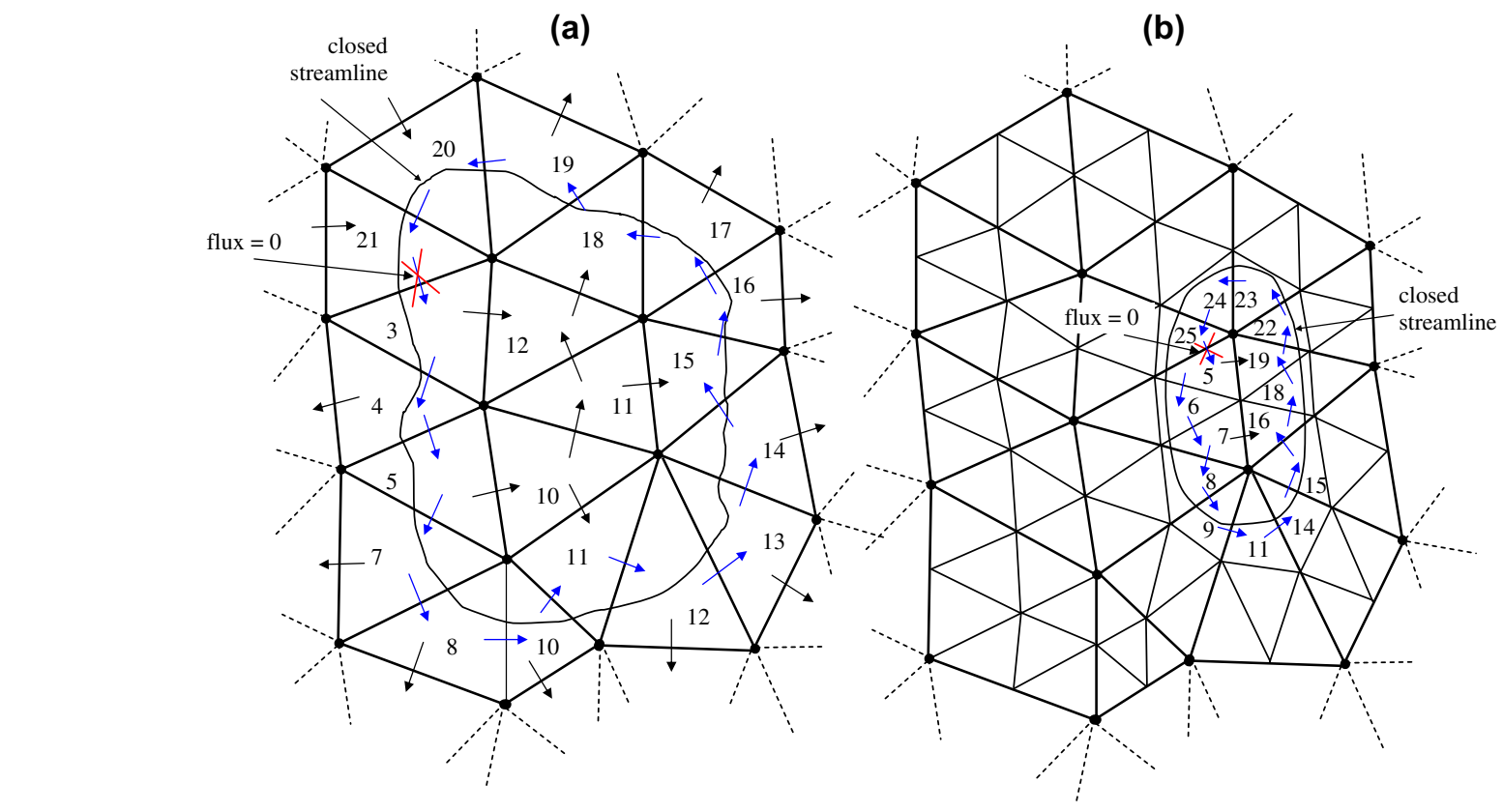

(b)

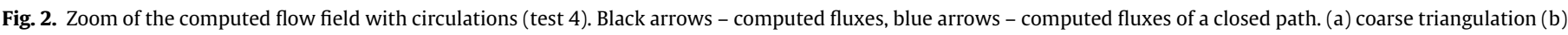

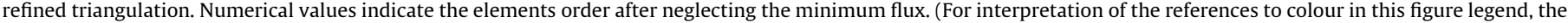
reader is referred to the web version of this article.)

$$
\begin{gathered}
\frac{\partial u h}{\partial t}+\frac{\partial}{\partial x}\left(u^{2} h\right)+\frac{\partial}{\partial y}(u v h)+g h \frac{\partial h}{\partial x} \\
+g h\left(\frac{\partial z}{\partial x}+\frac{n^{2} u \sqrt{(u h)^{2}+(v h)^{2}}}{h^{7 / 3}}\right) \\
=v\left(\frac{\partial}{\partial x}\left(h \frac{\partial u}{\partial x}\right)+\frac{\partial}{\partial y}\left(h \frac{\partial u}{\partial y}\right)\right), \\
\frac{\partial v h}{\partial t}+\frac{\partial}{\partial y}\left(v^{2} h\right)+\frac{\partial}{\partial x}(u v h)+g h \frac{\partial h}{\partial y} \\
+g h\left(\frac{\partial z}{\partial y}+\frac{n^{2} v \sqrt{(u h)^{2}+(v h)^{2}}}{h^{7 / 3}}\right) \\
=v\left(\frac{\partial}{\partial x}\left(h \frac{\partial v}{\partial x}\right)+\frac{\partial}{\partial y}\left(h \frac{\partial v}{\partial y}\right)\right),
\end{gathered}
$$

where $x$ and $y$ are the spatial coordinates $\left(x=x_{1}, y=x_{2}\right), t$ is the time, $u$ and $v$ are the $x$ and $y$ velocity components $\left(u=u_{1}, v=u_{2}\right), h$ is the water depth, $n$ is the Manning friction coefficient. The sum of the water depth and of the ground level, $H=z+h$, is the water level (or piezometric level or total head). Eqs. (15)-(17) represent respectively the mass and the $x$ and $y$ momentum conservation equations. The unknowns in system (15)-(17) are the water depth $h$ and the two flow rates components per unitary width in $x$ and $y$ directions, uh and $v h$.

\section{The MAST procedure}

\subsection{General formulation}

As mentioned in the introduction, MArching in Space and Time (MAST) solver is based on the following ideas [6-8]:

(a) splitting in each time step the original problem in a kinematic (prediction) problem plus a diffusive (correction) one. See in Appendix B more details of the fractional time step procedure, (b) solving the kinematic problem along the time step, one element after the other, moving in downstream direction of the scalar potential values, and solving the diffusive problem using a fully implicit formulation.

An appropriate ordering of the elements allows to cast the kinematic problem in each element as a small system of Ordinary Differential Equations (ODEs), that can be solved along a time step of any size without stability restrictions. The small size of the correction computed in the diffusive problem makes the artificial diffusion of its numerical solution small with respect to the size of the changes computed in the prediction step.

In the proposed algorithm the unknowns are computed in the circumcentre of each triangle, with a linear variation of the piezometric head inside each triangle and equal flux per unit width in the centre of the common side of two neighbour elements. Storage capacity is assumed concentrated in the circumcentre of each element, in the measure of the area of each triangle. The MAST scheme is suitable to higher order extension in both space and time $[5,6,10]$, but we believe that the natural heterogeneity and uncertainty of the parameters needed in the SWEs makes more suitable the 1st order approximation.

Spatial discretization of the governing PDEs is based on a generally unstructured triangular mesh. Let $\Omega \subset \mathfrak{R}^{2}$ be a bounded domain, $\Omega_{h}$ a polygonal approximation of $\Omega$ and $T_{h}$ an unstructured triangulation of $\Omega_{h}$. $N_{T}$ is the number of triangles of $T_{h}, T_{e}$, $e=1, \ldots, N_{T}$ is the generic triangle of $T_{h}$ and $\left|T_{e}\right|$ is the area of $T_{e}$. The computational mesh satisfies the generalised Delaunay (GD) condition (see details in Appendix C).

Call $i$, ip and im nodes of triangle $T_{e}$, where ip and im are the nodes respectively following and preceding node $i$ in counterclockwise direction. The edge vector $\mathbf{r}_{i, i p}\left(\mathbf{r}_{i, i m}\right)$ connects nodes $i$ and $i p$ (im), oriented from $i$ to ip (im). $T_{e p}$ is the triangle sharing side $\mathbf{r}_{i, i p}$ with $T_{e},\left(\mathbf{r}_{i p, i}=-\mathbf{r}_{i, i p}\right.$, oriented from ip to $\left.i\right) . c_{T_{e}}$ is the $T_{e}$ circumcentre with $\mathbf{x}_{c_{e}}$ its co-ordinate vector (see Fig. 3).

After integration of the prediction equations in space, applying the Green's theorem, the integral form of the prediction system is: 
$\frac{\partial h}{\partial t}\left|T_{e}\right|+\sum_{j=1,3} F_{j, e}=0, \ldots, e=1, \ldots, N T$,

$\frac{\partial u h}{\partial t}\left|T_{e}\right|+\sum_{j=1,3} M_{j, e}^{x}+R_{e}^{x}+\sum_{j=1,3} D_{j, e}^{x}=0$

$\frac{\partial v h}{\partial t}\left|T_{e}\right|+\sum_{j=1,3} M_{j, e}^{y}+R_{e}^{y}+\sum_{j=1,3} D_{j, e}^{y}=0$

where $F_{j, e}$ is the volumetric flux across side $j(j=1,2,3)$ of $T_{e}$, linking nodes $i$ and $i p\left(\mathbf{r}_{i, i p}\right)$ and $M_{j, e}^{x(y)}$ is the $x(y)$ component of the momentum flux along the same side. $F_{j, e}$ and $M_{j, e}^{x(y)}$ will be further specified. $R_{e}^{x}$ and $R_{e}^{y}$ are source terms defined as [7]:

$R_{e}^{x}=\left|T_{e}\right| g\left(h_{e} \frac{\partial H_{e}^{k}}{\partial x}+\frac{n^{2}(u h)_{e} \sqrt{(u h)_{e}^{2}+(v h)_{e}^{2}}}{h_{e}^{7 / 3}}\right)$,

$R_{e}^{y}=\left|T_{e}\right| g\left(h_{e} \frac{\partial H_{e}^{k}}{\partial y}+\frac{n^{2}(v h)_{e} \sqrt{(u h)_{e}^{2}+(v h)_{e}^{2}}}{h_{e}^{7 / 3}}\right)$,

with $H_{e}, h_{e}(u h)_{e}$ and $(v h)_{e}$ respectively the water level, the water depth and the flow rate components per unit width in element $T_{e}$. Finally, the viscous momentum flux components $D_{j, e}^{x}$ and $D_{j, e}^{y}$ are given respectively by:

$D_{j, e}^{X}=v h_{e} \int_{L_{j, e}} \frac{\partial u_{e}^{k}}{\partial n_{j, e}} \hat{\mathbf{n}}_{j, e} d l=v h_{e}\left|\mathbf{r}_{i, i p}\right| \frac{\partial u_{e}^{k}}{\partial n_{j, e}} \hat{\mathbf{n}}_{j, e}$

$D_{j, e}^{y}=v h_{e} \int_{L_{j, e}} \frac{\partial v_{e}^{k}}{\partial n_{j, e}} \hat{\mathbf{n}}_{j, e} d l=v h_{e}\left|\mathbf{r}_{i, i p}\right| \frac{\partial v_{e}^{k}}{\partial n_{j, e}} \hat{\mathbf{n}}_{j, e}$,

where $L_{j, e}$ marks the $j$ th side of element $T_{e}$ linking nodes $i$ and $i p$, with length $\left|\mathbf{r}_{i, i p}\right|$ and $\hat{\mathbf{n}}_{j, e}$ is its normal unit vector (positive outward). Spatial gradients of the velocity components in the $D_{j . e}^{x}$ and $D_{j, e}^{y}$ terms in Eq. (22) for triangle $T_{e}$ are computed by approximating:

$h_{e} \frac{\partial u_{e}^{k}}{\partial n_{j, e}} \hat{\mathbf{n}}_{j} \simeq h_{e} \frac{\partial}{\partial n_{j, e}}\left(\frac{(u h)_{e}}{h_{e}}\right)^{k} \hat{\mathbf{n}}_{j, e}$

$h_{e} \frac{\partial v_{e}^{k}}{\partial n_{j . e}} \hat{\mathbf{n}}_{j} \simeq h_{e} \frac{\partial}{\partial n_{j . e}}\left(\frac{(v h)_{e}}{h_{e}}\right)^{k} \hat{\mathbf{n}}_{j, e}$

and the derivatives at the r.h.s. of Eq. (23) are computed assuming a linear variation between the values of $\frac{(u h)}{h}$ and $\frac{(v h)}{h}$ at time level $t^{k}$ in triangles $T_{e}$ and $T_{e p}$ sharing side $\mathbf{r}_{i, i p}$. More details on the computation of the spatial piezometric head gradients are given in Section 4.3.

According to the formulation in Eqs. (B.3)-(B.5) of Appendix B, the differential linearized form of the correction problem is:

$\frac{\partial h}{\partial t}+\frac{\partial u h}{\partial x}+\frac{\partial v h}{\partial y}=\frac{\partial(\overline{u h})}{\partial x}+\frac{\partial(\overline{v h})}{\partial y}$,

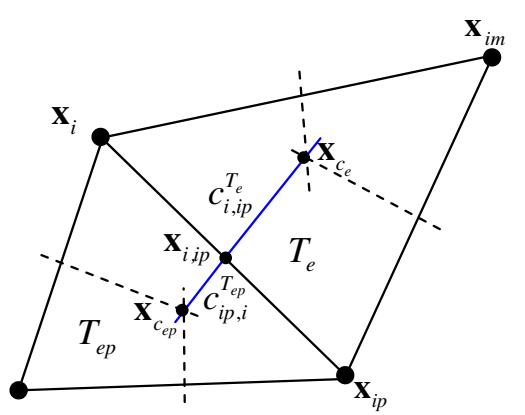

Fig. 3. Elements notation.

$$
\begin{array}{r}
\frac{\partial u h}{\partial t}+g \bar{h} \frac{\partial H}{\partial x}+g n^{2}(u h) \overline{\left(\frac{\sqrt{(u h)^{2}+(v h)^{2}}}{h^{7 / 3}}\right)} \\
=g \bar{h} \frac{\partial H^{k}}{\partial x}+g n^{2}(\overline{u h}) \overline{\left(\frac{\sqrt{(u h)^{2}+(v h)^{2}}}{h^{7 / 3}}\right)} \\
\frac{\partial v h}{\partial t}+g \bar{h} \frac{\partial H}{\partial y}+g n^{2}(v h) \overline{\left(\frac{\sqrt{(u h)^{2}+(v h)^{2}}}{h^{7 / 3}}\right)} \\
=g \bar{h} \frac{\partial H^{k}}{\partial y}+g n^{2}(\overline{v h})\left(\frac{\sqrt{(u h)^{2}+(v h)^{2}}}{h^{7 / 3}}\right)
\end{array}
$$

where the over bar symbol marks the corresponding mean in time values, computed as explained in the next sections. Initial conditions of the correction system are the final values of the prediction system.

In Eqs. (25) and (26) we neglect the difference between the sum of inertial and viscous flux terms and the corresponding mean in time value computed from the solution of the prediction system $[6,7]$. This is equivalent to assume, in the correction system:

$$
\begin{aligned}
& \frac{\partial}{\partial x}\left(u^{2} h\right)+\frac{\partial}{\partial y}(u v h) \simeq \frac{\partial}{\partial x}\left(\overline{u^{2} h}\right)+\frac{\partial}{\partial y}(\overline{u v h}) \\
& \frac{\partial}{\partial y}\left(v^{2} h\right)+\frac{\partial}{\partial x}(u v h) \simeq \frac{\partial}{\partial y}\left(\overline{v^{2} h}\right)+\frac{\partial}{\partial x}(\overline{u v h}) \\
& v\left(\frac{\partial}{\partial x}\left(h \frac{\partial u}{\partial x}\right)+\frac{\partial}{\partial y}\left(h \frac{\partial u}{\partial y}\right)\right) \simeq v\left(\frac{\partial}{\partial x}\left(\bar{h} \frac{\partial \bar{u}}{\partial x}\right)+\frac{\partial}{\partial y}\left(\bar{h} \frac{\partial \bar{u}}{\partial y}\right)\right) \\
& v\left(\frac{\partial}{\partial x}\left(h \frac{\partial v}{\partial x}\right)+\frac{\partial}{\partial y}\left(h \frac{\partial v}{\partial y}\right)\right) \simeq v\left(\frac{\partial}{\partial x}\left(\bar{h} \frac{\partial \bar{v}}{\partial x}\right)+\frac{\partial}{\partial y}\left(\bar{h} \frac{\partial \bar{v}}{\partial y}\right)\right) .
\end{aligned}
$$

\subsection{The prediction problem}

Triangles $T_{e}$ and $T_{e p}$ share side $\mathbf{r}_{i, i p}$ between nodes $i$ and $i p . \mathbf{r}_{i, i p}$ is the $j$ th side of $T_{e}$ and $\mathbf{r}_{i p, i}$ is the $m$ th side of $T_{e p}(j, m=1,2,3)$. The volumetric flux across side $j$ of $T_{e}$ is equal to [7]:

$F L_{j, e}=(u h)_{e}\left(y_{i p}-y_{i}\right)-(v h)_{e}\left(x_{i p}-x_{i}\right)$.

According to Eq. (28) the leaving fluxes are positive, the entering ones negative. We finally define the volumetric flux between $T_{e}$ and $T_{e p}$ as [7]:

$F_{j, e}=F L_{j, e} \quad$ if $F L_{i, i p}^{e}>0$ and $F L_{j, e}>F L_{m, e p}$,

$F_{j, e}=-F L_{m, e p} \quad$ otherwise,

$M_{j, e}^{x}=F_{j, e} u_{e}, \quad M_{j, e}^{y}=F_{j, e} v_{e} \quad$ if $F_{j, e}=F L_{j, e}$,

$M_{j, e}^{x}=F_{j, e} u_{e p}, \quad M_{j, e}^{y}=F_{j, e} v_{e p} \quad$ otherwise.

Condition $F_{j, e}=-F_{m, e p}$ holds for all the internal sides. If $F_{e, j}$ is the positive (outward oriented) flux of an external boundary side, condition $F_{j, e}=F L_{j, e}$ holds. On the base of Eqs. (29) and (30), volumetric flux and momentum flux continuity is always guaranteed for each internal element side.

According to formulations given in Eqs. (28)-(30) and to the element ordering procedure presented in Section 2, flux and momentum fluxes from $T_{e}$ to $T_{e p}$ in the prediction step are only function of the $T_{e}$ unknowns if $n o_{e}^{k}<n o_{e p}^{k}$ and are only function of the $T_{e p}$ unknowns if $n o_{e}^{k}>n o_{e p}^{k}$. Due to the assumption of a constant (in time) total head gradient in the prediction step, the prediction system (18)-(20) can be solved as an Ordinary 
Differential Equations (ODEs) system. We solve the prediction step as a sequence of small ODEs systems, one for each computational element, after ordering the elements according to the procedure proposed in Section 2. The ODEs system for the generic triangle $T_{e}$ is given by:

$$
\begin{aligned}
\frac{d h_{e}}{d t}\left|T_{e}\right| & +\frac{1}{\Delta t} \sum_{j=1,3} \delta_{j, e} \int_{\Delta t} F_{j, e}^{o u t} d t \\
& =\frac{1}{\Delta t} \sum_{j=1,3}\left(1-\delta_{j, e}\right) \int_{\Delta t} F_{j, e}^{i n} d t \quad e=1, \ldots, N_{T},
\end{aligned}
$$

$$
\begin{aligned}
& \frac{d(u h)_{e}}{d t}\left|T_{e}\right|+\frac{1}{\Delta t}\left(\left(\sum_{j=1,3} \delta_{j, e} \int_{\Delta t} M_{j, e}^{x, \text { out }} d t\right)+\int_{\Delta t} R_{e}^{x} d t+\sum_{j=1,3} \int_{\Delta t} D_{j, e}^{x} d t\right) \\
& =\frac{1}{\Delta t} \sum_{j=1,3}\left(1-\delta_{j, e}\right) \int_{\Delta t} M_{j, e}^{x, i n} d t
\end{aligned}
$$

$$
\begin{aligned}
& \frac{d(v h)_{e}}{d t}\left|T_{e}\right|+\frac{1}{\Delta t}\left(\left(\sum_{j=1,3} \delta_{j, e} \int_{\Delta t} M_{j, e}^{y, \text { out }} d t\right)+\int_{\Delta t} R_{e}^{y} d t+\sum_{j=1,3} \int_{\Delta t} D_{j, e}^{y} d t\right) \\
& =\frac{1}{\Delta t} \sum_{j=1,3}\left(1-\delta_{j, e}\right) \int_{\Delta t} M_{j, e}^{y, \text { in }} d t
\end{aligned}
$$

where $\delta_{j, e}=1$ or 0 if flux across side $j$ is oriented outward $T_{e}$ or not, $R_{e}^{x(y)}$ and $D_{j, e}^{x(y)}$ are defined respectively in Eq. (21) and in Eq. (22) and indices in and out mark the fluxes and momentum fluxes oriented inward and outward element $T_{e}$ respectively. Viscous momentum fluxes components appear in Eqs. (32) and (33) respect to the previous formulation in [7].

The solution of the ODEs system is further simplified if we change the r.h.s. of each equation with its mean value along the given time step, according to:

$$
\begin{aligned}
& \frac{d h_{e}}{d t}\left|T_{e}\right|+\frac{1}{\Delta t} \sum_{j=1,3} \delta_{j, e} \int_{\Delta t} F_{i, i p}^{e, o u t} d t=\overline{F_{e}^{i n}} \quad e=1, \ldots, N_{T} \\
& \frac{d(u h)_{e}}{d t}\left|T_{e}\right|+\frac{1}{\Delta t}\left(\left(\sum_{j=1,3} \delta_{j, e} \int_{\Delta t} M_{i, i p}^{e, x, o u t} d t\right)+\int_{\Delta t} R_{e}^{x} d t+\sum_{j=1,3} \int_{\Delta t} D_{i, i p}^{e, x} d t\right) \\
& =\overline{M_{e}^{x, \text { in }}}
\end{aligned}
$$

$$
\begin{aligned}
& \frac{d(v h)_{e}}{d t}\left|T_{e}\right|+\frac{1}{\Delta t}\left(\left(\sum_{j=1,3} \delta_{j, e} \int_{\Delta t} M_{i, i p}^{e, y, o u t} d t\right)+\int_{\Delta t} R_{e}^{y} d t+\sum_{j=1,3} \int_{\Delta t} D_{i, i p}^{e, y} d t\right) \\
& =\overline{M_{e}^{y, i n}}
\end{aligned}
$$

where the r.h.s. of Eqs. (34)-(36), that is the mean in time values of the incoming volumetric fluxes and momentum fluxes, are known from the solution of the previously solved elements, as further specified.Elements are ordered at the beginning of the time step according to the fluxes computed across their sides, applying the ordering procedure described in Section 2. Systems (34)-(36) are then solved sequentially, one after the other, proceeding from the lowest to the highest ordering number. Elements with the same order number can be solved independently of each other and an element with a given order can be solved only after the solution of the neighbouring ones with lower order. Element solution is function of the initial state in the same element and of the fluxes and momentum fluxes incoming from the already solved neighbouring elements with lower order. For this reason the prediction step can be regarded as the "explicit" component of the algorithm. The ODEs system is solved along the original time step using a variable step Runge-Kutta method with adaptive stepsize control $[7,36]$. Mean in time values $\bar{h}$ and $\sqrt{\frac{(u h)^{2}+(v h)^{2}}{h^{7 / 3}}}$, required in the correction step, are computed via numerical integration according to a $C^{1}$ interpolation of the solution values computed at Gauss points, selected in the time interval $\left[t^{k}-t^{k+1}\right][6,7]$. Mean in time values of $u h$ and $v h$ are computed in a different way, after integration in space, in order to guarantee the mass balance for the element.After the ODEs in element $T_{e}$ are solved, the mean total flux $\overline{F_{e}^{\text {out }}}$ leaving from $T_{e}$ along the time step is computed from the local mass balance [7]. Once the total mean leaving flux is computed, the mean flux $\overline{F_{j, e}^{\text {out }}}$ leaving from side $\mathbf{r}_{i, i p}$ of $T_{e}$ to the neighbouring element $T_{e p}$ with $n o_{e}^{k}<n o_{e p}^{k}$, can be estimated by partitioning $\overline{F_{e}^{\text {out }}}$ according to the ratio between the flux $F_{j, e}^{\text {out }}$ and the sum of the leaving fluxes at the end of the time step (details in [7]). Mean leaving momentum fluxes $\overline{M_{j, e}^{x, \text { out }}}$ and $\overline{M_{j, e}^{y, \text { out }}}$ can also be estimated in a similar way [7]. Finally you set:

$\overline{F_{m, e p}^{\text {in }}}=\overline{F_{j, e}^{\text {out }}} \quad \overline{M_{m, e p}^{x, i n}}=\overline{M_{j, e}^{x, \text { out }}} \quad \overline{M_{m, e p}^{y, \text { in }}}=\overline{M_{j, e}^{y, \text { out }}}$

for all the neighbouring $T_{e p}$ elements with $n o_{e}^{k}<n o_{e p}^{k}$ and you can proceed to solve system (34)-(36) for the next element, that has among the unsolved ones the minimum number of order greater than or equal to $n o_{e}^{k}$.

Conservation of the mean values can be easily proved to guarantee the local and global mass conservation [6,7] and the proof of the local and global mass conservation of the prediction step is given in [8].

\subsection{The correction problem}

A fully implicit time discretization is adopted for the solution of the diffusive correction problem (24)-(26). It leads for the generic element $T_{e}$ to:

$$
\begin{aligned}
& \frac{H_{e}^{k+1}-H_{e}^{k+\frac{1}{2}}}{\Delta t}+\frac{\partial(u h)_{e}^{k+1}}{\partial x}+\frac{\partial(v h)_{e}^{k+1}}{\partial y}=\frac{\partial \overline{(u h)_{e}}}{\partial x}+\frac{\partial \overline{(v h)_{e}}}{\partial y} \\
& \frac{(u h)_{e}^{k+1}-(u h)_{e}^{k+\frac{1}{2}}}{\Delta t}+g \bar{h}_{e} \frac{\partial H_{e}^{k+1}}{\partial x} \\
& +g n^{2}(u h)_{e}^{k+1} \overline{\left(\frac{\sqrt{(u h)_{e}^{2}+(v h)_{e}^{2}}}{h_{e}^{7 / 3}}\right)} \\
& =g \bar{h}_{e} \frac{\partial H_{e}^{k}}{\partial x}+g n^{2} \overline{(u h)_{e}} \overline{\left(\frac{\sqrt{(u h)_{e}^{2}+(v h)_{e}^{2}}}{h_{e}^{7 / 3}}\right)}, \\
& \frac{(v h)_{e}^{k+1}-(v h)_{e}^{k+\frac{1}{2}}}{\Delta t}+g \bar{h}_{e} \frac{\partial H_{e}^{k+1}}{\partial y} \\
& +g n^{2}(v h)_{e}^{k+1} \overline{\left(\frac{\sqrt{(u h)_{e}^{2}+(v h)_{e}^{2}}}{h_{e}^{7 / 3}}\right)} \\
& =g \bar{h}_{e} \frac{\partial H_{e}^{k}}{\partial y}+g n^{2} \overline{(v h)_{e}} \overline{\left(\frac{\sqrt{(u h)_{e}^{2}+(v h)_{e}^{2}}}{h_{e}^{7 / 3}}\right)}
\end{aligned}
$$

with the above specified symbols, where index $k+1 / 2$ marks the values of $H, u h$ and $v h$ computed at the end of the prediction step. From Eqs. (39) and (40) one gets:

$$
\begin{aligned}
& (u h)_{e}^{k+1}=- \text { elem }_{e} \frac{\partial H_{e}^{k+1}}{\partial x}+k_{e}^{x}+(u h)_{e}^{k+1 / 2} \\
& (v h)_{e}^{k+1}=- \text { elem }_{e} \frac{\partial H_{e}^{k+1}}{\partial y}+k_{e}^{y}+(v h)_{e}^{k+1 / 2},
\end{aligned}
$$


with elem $_{e}=\frac{g \bar{h}_{e} \Delta t}{1+\Delta \operatorname{tgn} n^{2}\left(\frac{\sqrt{(u h)_{e}^{2}+(v h)_{e}^{2}}}{h_{e}^{7 / 3}}\right)}, \quad k_{e}^{x}=\operatorname{elem}_{e} \frac{\partial H_{e}^{k}}{\partial x}$

$k_{e}^{y}=\operatorname{elem}_{e} \frac{\partial H_{e}^{k}}{\partial y}$.

After space integration, merging Eqs. (41) and (42) in Eq. (38) and applying the Green theorem, one gets the following balance law for triangle $T_{e}$ :

$$
\begin{aligned}
& \int_{T_{e}} \frac{\partial H}{\partial t} d T_{e}+\sum_{j=1,3} \int_{L_{j, e}}-\text { elem }_{e} \frac{\partial H^{k+1}}{\partial n_{j, e}} d l \\
& \quad=\sum_{j=1,3} \int_{L_{j, e}}-\operatorname{elem}_{e} \frac{\partial H^{k}}{\partial n_{j, e}} d l+\sum_{j=1,3} \int_{L_{j, e}}\left(\overline{\mathbf{q}}-\mathbf{q}^{k+1 / 2}\right) \cdot \hat{\mathbf{n}}_{j, e} d l,
\end{aligned}
$$

where $\overline{\mathbf{q}}$ and $\mathbf{q}^{k+1 / 2}$ are respectively the mean in time and the final values of the specific flow rate vector computed after the solution of the prediction step. Sum of fluxes due to $\overline{\mathbf{q}}$ is computed according to the mass balance for $T_{e}$ :

$\sum_{j=1,3} \int_{L_{j}} \overline{\mathbf{q}} \cdot \hat{\mathbf{n}}_{j, e} d l=\overline{F_{e}^{i n}}-\overline{F_{e}^{o u t}}=\frac{H_{e}^{k+1 / 2}-H_{e}^{k}}{\Delta t}\left|T_{e}\right|$,

while the corresponding term due to $\mathbf{q}^{k+1 / 2}$ is obtained by summing the fluxes given by Eqs. (28) and (29) using the final prediction step solution. After time discretization, Eq. (43) can be written as:

$\frac{H_{e}^{k+1}-H_{e}^{k+1 / 2}}{\Delta t}\left|T_{e}\right|+\sum_{j=1,3} \tilde{F}_{j, e}=\sum_{j=1,3} \tilde{b}_{j, e}$,

where the flux $\tilde{F}_{j, e}$ across side $j$ of $T_{e}$ linking nodes $i$ and $i p\left(\mathbf{r}_{i, i p}\right)$ is:

$\tilde{F}_{j, e}=-\operatorname{elem}_{e} \frac{\partial H^{k+1}}{\partial n_{j, e}}\left|\mathbf{r}_{i, i p}\right|$

and the source term $\tilde{b}_{j, e}$ is:

$$
\begin{aligned}
\tilde{b}_{j, e}= & \left((\overline{u h})_{e}-(u h)_{e}^{k+1 / 2}-k_{e}^{x}\right)\left(y_{i p}-y_{i}\right)-\left((\overline{v h})_{e}-(v h)_{e}^{k+1 / 2}\right. \\
& \left.-k_{e}^{y}\right)\left(x_{i p}-x_{i}\right) .
\end{aligned}
$$

The total head derivatives in Eq. (46,a) are discretized according to the MHFE scheme lumped in the elements circumcentres, proposed in [9]. This formulation leads to:

$\tilde{F}_{j, e}=\chi_{j, e}\left(H_{e}^{k+1}-H_{i, i p}^{k+1}\right)$,

with coefficient $\chi_{j, e}$ given by Aricò et al. [9]:

$\chi_{j, e}=\frac{\text { elem }_{e}}{c_{i, i p}^{T_{e}}}\left|\mathbf{r}_{i, i p}\right|$

where $c_{i, i p}^{T_{e}}$ is the distance between the $T_{e}$ circumcentre $c_{T_{e}}$ and the midpoint of $\mathbf{r}_{i, i p}$, computed as in Eq. (C.1) of Appendix C. Identity of fluxes between elements $T_{e}$ and $T_{e p}$ across their common side $\mathbf{r}_{i, i p}$ provides, after some simple algebraic manipulations:

$\tilde{F}_{j, e}=\bar{\chi}_{e, e p}\left(H_{e}^{k+1}-H_{e p}^{k+1}\right)$,

where flux coefficient $\bar{\chi}_{e, e p}$ given by Aricò et al. [9]:

$\bar{\chi}_{e, e p}=\frac{\chi_{j, e} \chi_{m, e p}}{\chi_{j, e}+\chi_{m, e p}}=\frac{\left|\mathbf{r}_{i, i p}\right|}{\frac{c_{i, p}^{T_{e}}}{\text { eleme }_{e}}+\frac{c_{i p, i}^{T_{e p}}}{\text { elemep }_{e p}}}$.

Such a formulation guarantees, as in the prediction problem, flux continuity at element interfaces. Eq. (45) form a linear system of order $N_{T}$ in the $H_{e}\left(e=1, \ldots, N_{T}\right)$ unknowns with fully implicit time discretization. Diagonal term of the stiffness matrix system corresponding to element $T_{e}$ is: $s_{e, e}=\frac{\left|T_{e}\right|}{\Delta t}+\sum_{e p=1 . N_{T}} \bar{\chi}_{e, e p} \delta_{e, e p}$

where $\delta_{e, e p}=1$ if elements $T_{e}$ and $T_{e p}$ share a side, otherwise it is zero and its off-diagonal term corresponding to triangle $T_{e p}$ is:

$s_{e, e p}=-\hat{\chi}_{e, e p}$.

According to the flux coefficient formulation given in Eq. (50), offdiagonal coefficients for obtuse triangles could be non negative and $M$-matrix property would be lost also for a generalised Delaunay mesh with positive sum of distances $c_{i, i p}^{T_{e}}+c_{i p, i}^{T_{e p}}$ (see Eq. (C.2) in Appendix C), if the two coefficients $\chi_{i, i p}^{e}$ and $\chi_{i p, i}^{e p}$ were computed with different element parameters elem end $_{e}$ alemep. In this case, the sign of the total flux from $T_{e}$ to $T_{e p}$ can loose consistency with the $H$ difference. Given a generalised Delaunay mesh, we propose the following formulation for coefficient $\bar{\chi}_{e, e p}[9]$ :

$\bar{\chi}_{e, e p}=\min \left(\operatorname{big}, \frac{\left|\mathbf{r}_{i, i p}\right|}{\frac{c_{e}}{\text { eleme }_{e}}+\frac{c_{e p}}{\text { elemep }}}\right)$,

where $c_{e}$ and $c_{e p}$ are defined as:

$c_{e}=c_{i, i p}^{T_{e}} \quad c_{e p}=c_{i p, i}^{T_{e p}} \quad$ if $c_{i, i p}^{T_{e}}>0, c_{i p, i}^{T_{e p}}>0$,
$c_{e}=c_{i, i p}^{T_{e}}+c_{i p, i}^{T_{e p}} \quad c_{e}=0 \quad$ if $c_{i, i p}^{T_{e}}>0, c_{i p, i}^{T_{e p}} \leqslant 0 \quad$ and $\quad\left|c_{i p, i}^{T_{e p}}\right|<c_{i, i p}^{T_{e}}$,
$c_{e}=0 \quad c_{e p}=c_{i, i p}^{T_{e}}+c_{i p, i}^{T_{e p}} \quad$ if $c_{i p, i}^{T_{e p}}>0, c_{i, i p}^{T_{e}} \leqslant 0 \quad$ and $\quad\left|c_{i, i p}^{T_{e}}\right|<c_{i p, i}^{T_{e p}}$

and big is a very large positive number (say big $\simeq 1 . d+15$ ). Formulation provided by Eq. (52) always guarantees for GD meshes the negative sign of the off-diagonal coefficient defined by Eq. $(51, b)$, along with the $M$-property and the positive definite condition.

Observe that the flux formulation between the two elements $T_{e}$ and $T_{e p}$ given in Eq. (49) using coefficient $\bar{\chi}_{e, e p}$, modified according to Eq. (52), is consistent with the geometry of the Delaunay mesh. If the two triangles sharing side $\mathbf{r}_{i, i p}$ are acute triangles, formulations (50) and (52) overlap; if one of the two triangles is obtuse, the flux computed according to formulations (52) is still equal to the flux through side $\mathbf{r}_{i, i p}$, due to a $H$ gradient between the two $T_{e}$ and $T_{e p}$ triangles circumcentres, computed according to the coefficient elem of the acute triangle where the segment between $c_{T_{e}}$ and $c_{T_{e p}}$ is entirely located (see Fig. 4(a)). In this case, the flux computed with the coefficients given by the original Eq. (50) is different and could not be consistent with the velocity occurring in the acute triangle.

Once system (45) has been solved, the new piezometric head gradients $\frac{\partial H_{e}^{k+1}}{\partial x}$ and $\frac{\partial H_{e}^{k+1}}{\partial y}$ are computed in each element according to the three midpoint values. We distinguish two different cases for each side $\mathbf{r}_{i, i p}$.

(1) $\mathbf{r}_{i, i p}$ is a generic internal side. Midpoint value $H_{i, i p}^{k+1}$ is obtained by comparing Eqs. (47) and (49), where $H_{e}^{k+1}$ and $H_{e p}^{k+1}$ are known, to get:

$H_{i, p}^{k+1}=\frac{\left(H_{e}^{k+1} \chi_{j, e}+H_{e p}^{k+1} \chi_{m, e p}\right)}{\chi_{j, e}+\chi_{m, e p}}$

with the above specified symbols for coefficients $\chi_{j, e}$ and $\chi_{m, e p}$. After simple algebraic manipulations, Eq. (53) is written as:

$H_{i, i p}^{k+1}=\frac{H_{e}^{k} c_{e p} \text { elem }_{e p}+H_{e p}^{k} c_{e} \text { elem }_{e}}{c_{e} \text { elem }_{e p}+c_{e p} \text { elem }_{e}}$ if $\left(c_{e}\right.$ elem $_{e p}+c_{e p}$ elem $\left._{e}\right) \geqslant$ toll

$H_{i, i p}^{k+1}=\frac{H_{e}^{k}+H_{e p}^{k}}{2} \quad$ if $\left(c_{e} e l e m_{e p}+c_{e p}\right.$ elem $\left.{ }_{e}\right)<$ toll, 
where toll is the machine precision and distances $c_{e}$ and $c_{e p}$ are computed by Eq. (52,b).

(2) $\mathbf{r}_{i, i p}$ is a boundary side. $H_{i, i p}$ is computed according to the boundary conditions specified in the following section.

After computation of the new gradients $\frac{\partial H_{e}^{k+1}}{\partial x}$ and $\frac{\partial H_{e}^{k+1}}{\partial y}$, specific flow rate components at the end of the correction problems $(u h)_{e}^{k+1}$ and $(v h)_{e}^{k+1}$ are obtained by Eqs. (41) and (42). The values of the new computed gradients will be kept constant during the solution of the correction step of the next time iteration. The piezometric head gradient formulation in Eqs. (53) and (54) is completely different form the ones suggested in the previous work [7], where two distinguished computations have been carried out for the convective and diffusive steps. Formulation suggested in Eqs. (53) and (54) is coherent with the numerical procedure proposed in this section which guarantees the $M$-property of the system matrix.

Once specific flow rates are updated, the gradients $\frac{\partial}{\partial n_{j, e}}\left(\frac{(u h)_{e}}{h_{e}}\right)$ and $\frac{\partial}{\partial n_{j, e}}\left(\frac{(u h)_{e}}{h_{e}}\right)$ can be computed for the new time iteration according to the new circumcentre values.

\subsection{Boundary conditions}

Let $T_{e}$ be a boundary element and its $j$ th side $\left(\mathbf{r}_{i, i p}\right)$ a boundary side. Let $(u h)_{e}^{k}$ and $(v h)_{e}^{k}$ the specific flow rate components computed inside $T_{e}$ at the beginning of a time step (time level $t^{k}$ ). Moreover, we distinguish the external assigned values of water depths and specific flow rate components, $h_{e}^{e x},(u h)_{e}^{e x}$ and $(v h)_{e}^{e x}$, from the corresponding boundary side values, $h_{e}^{b},(u h)_{e}^{b}$ and $(v h)_{e}^{b}$. At the beginning of each time step we compute for each boundary element side the volumetric flux $\mathrm{Fl}_{\text {j,e }}$ (as in Eq. (28)) and the Froude number $f r_{j, e}^{k}$ of the flux per unit length as:

$f r_{j, e}^{k}=\frac{(u h)_{e}^{b}\left(y_{i p}^{e}-y_{i}^{e}\right)-(v h)_{e}^{b}\left(x_{i p}^{e}-x_{i}^{e}\right)}{\left|\mathbf{r}_{i, i p}\right|\left(h_{e}^{b}\right)^{3 / 2} \sqrt{g}}$,

with the above specified symbols. The boundary water depth $h_{e}^{b}$ is linked to the midpoint water level $H_{i, i p}^{k}$ at the beginning of the time step by the relationship:

$h_{e}^{b}=H_{i, i p}^{k}-z_{j, e}$,

where $z_{j, e}$ is the topographic level of the midpoint of the boundary side and $H_{i, i p}^{k}$ is equal to the corresponding midpoint value computed at the end of the previous time step, as further explained.

One of the following cases occurs:

(1) $f r_{j, e}^{k}>1$ and $F l_{j, e}$ entering the domain $\left(F L_{j, e}<0\right)$. In the prediction problem the incoming volumetric and momentum fluxes are known and equal to:
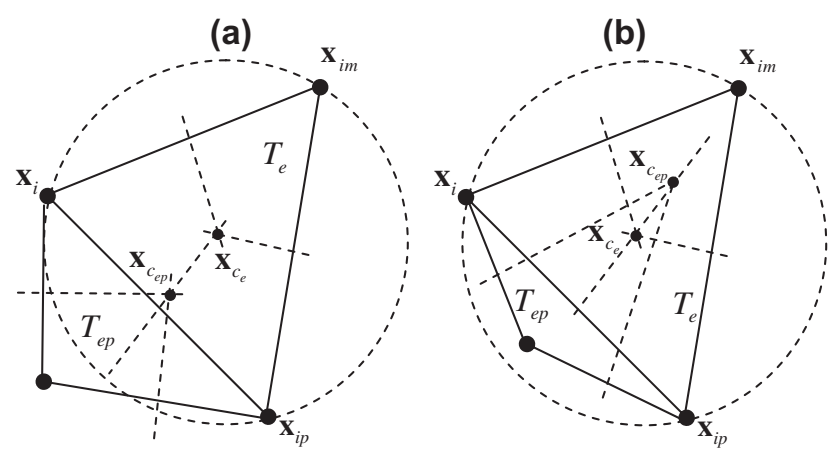

Fig. 4. (a) Side $\mathbf{r}_{i, i p}$ satisfies Delaunay property. (b) Side $\mathbf{r}_{i, i p}$ does not satisfy Delaunay property.
$F_{e, j}=(u h)_{e}^{b}\left(y_{i p}^{e}-y_{i}^{e}\right)-(v h)_{e}^{b}\left(x_{i p}^{e}-x_{i}^{e}\right)$

$M_{e, j}^{x}=F_{e, j} \frac{(u h)_{e}^{b}}{h_{e}^{b}}$

$M_{e, j}^{y}=F_{e, j} \frac{(v h)_{e}^{b}}{h_{e}^{b}}$,

with $(u h)_{e}^{b}=(u h)_{e}^{e x}(v h)_{e}^{b}=(v h)_{e}^{e x} \quad h_{e}^{b}=h_{e}^{e x}$.

In the correction problem $H_{i, i p}^{k+1}$ is assumed equal to the Dirichlet assigned value, i.e. $H_{i, i p}^{k+1}=h_{e}^{e x}+z_{j, e}$. Observe that $H_{e}^{k+1}$ remains an unknown of the correction system and a flux given by Eq. (47) has to be added in the 1.h.s. of Eq. (45) corresponding to element $T_{e}$. Boundary side values $h_{e}^{b}$, $(u h)_{e}^{b}$ and $(v h)_{e}^{b}$ in Eq. (55) for the next time step are given by Eq. $(57, \mathrm{~b})$.

(2) $f r_{j, e}^{k}>1$ and $F l_{j, e}$ leaving the domain $\left(F l_{j, e} \geqslant 0\right)$. No boundary condition is required in this case. The ODEs system of the prediction problem is solved for element $T_{e}$ as described above. In the correction step call $F_{j, e}^{c}$ the corrective flux, given by (see Eq. (46)):

$F_{j, e}^{c}=\tilde{F}_{j, e}+\tilde{b}_{j, e}$

Set $F_{j, e}^{c}=0$ in Eq. (45) corresponding to element $T_{e}$. After solution of the correction system compute $H_{i, i p}^{k+1}$ by merging Eqs. (47) and (58), to get:

$\chi_{j, e}\left(H_{e}^{k+1}-H_{i, i p}^{k+1}\right)=-\tilde{b}_{j, e}$.

Boundary side value $h_{e}^{b}$ in Eq. (55) for the next time iteration is computed by Eq. (56), while $(u h)_{e}^{b}$ and $(v h)_{e}^{b}$ are equal to the element values computed at the end of the correction problem, respectively $(u h)_{e}^{k+1}$ and $(v h)_{e}^{k+1}$.

(3) $f r_{j, e}^{k} \leqslant 1$ and $F l_{j, e}$ entering the domain $\left(F L_{j, e}<0\right)$. In this case we assume the specific discharge components $(u h)_{e}^{b}$ and $(v h)_{e}^{b}$ to be known and equal respectively to $(u h)_{e}^{e x}$ and $(v h)_{e}^{e x}$. In the prediction step, volumetric and momentum fluxes are computed using the known discharge components $(u h)_{e}^{b}$ and $(v h)_{e}^{b}$ and the boundary water depth $h_{e}^{b}$ computed at the end of the previous time step. In the correction step, zero corrective flux is assigned $\left(F_{j . e}^{c}=0\right)$, as explained for the previous case (2) and the midpoint $H_{i, i p}^{k+1}$ value is computed accordingly. Boundary side value $h_{e}^{b}$ in Eq. (55) for the next time iteration is computed by Eq. (56).

(4) $f r_{j, e}^{k} \leqslant 1$ and $F l_{j, e}$ leaving the domain $\left(F l_{j, e} \geqslant 0\right)$. No special treatment is needed for element $T_{e}$ in the prediction step. Let $(u h)_{e}^{b}$ and $(v h)_{e}^{b}$ be equal respectively to $(u h)_{e}^{k+1 / 2}$ and $(v h)_{e}^{k+1 / 2}$, the element values computed at the end of the prediction step. In the correction step two possibilities exist. If the assigned external water depth $h_{e}^{e x}$ is smaller than the critical depth $h_{e}^{c}$ corresponding to the specific flow rate on the boundary side, that is:

$h_{e}^{e x} \leqslant h_{e}^{c}$ with $h_{e}^{c}=\left(\frac{\left((u h)_{e}^{b}\right)^{2}+\left((v h)_{e}^{b}\right)^{2}}{g}\right)^{1 / 3}$

a corrective flux corresponding to the critical depth inside the element is assigned to the element boundary side, equal to:

$F_{e, j}^{c}=\sqrt{g}\left(h_{e}^{c}\right)^{3 / 2}\left|\mathbf{r}_{i, i p}\right|-F_{e, j}^{k+1 / 2}$,

where $F_{e, j}^{k+1 / 2}$ is given by Eq. (28) at the end of the prediction step. After solution of the correction system, $H_{i, i p}^{k+1}$ is computed as solution of Eq. (47), written as:

$\chi_{j, e}\left(H_{e}^{k+1}-H_{i, i p}^{k+1}\right)+\tilde{b}_{j, e}=F_{e, j}^{c}$

with $F_{j, e}^{c}$ given by Eq. $(60, b)$. If constraint $(60, a)$ does not hold, the external water depth is assigned in the midpoint of the boundary 
side as Dirichlet value, as already explained for case (1). In both cases, $h_{e}^{b}$ for the next time iteration is computed by Eq. (56), while the boundary values $(u h)_{e}^{b}$ and $(v h)_{e}^{b}$ are assumed equal respectively to $(u h)_{e}^{k+1}$ and $(v h)_{e}^{k+1}$.

\subsection{Model properties}

The model preserves the $C$-property (see for example [46]). For quiescent water, in facts, we have, in the prediction step, zero flux entering in each element and zero gradient of the piezometric head. This implies, in the solution of system (34)-(36), $H^{k+1 /}$ ${ }^{2}=H^{k}$. In the correction step we solve system (43) which, after simple manipulations, can be written as:

$\int_{T_{e}} \frac{\partial \eta}{\partial t}-\sum_{j=1,3} \int_{L_{j, e}}\left(\operatorname{elem}_{e} \frac{\partial(\eta-\vartheta)}{\partial n_{j, e}}\right)=0$

with $\eta=H-H^{k+1 / 2} \vartheta=H^{k}-H^{k+1 / 2}$,

where $\vartheta$ is zero from the solution of the prediction step. System (61) becomes:

$\int_{T_{e}} \frac{\partial \eta}{\partial t}-\sum_{j=1,3} \int_{L_{j, e}}\left(\operatorname{elem}_{e} \frac{\partial \eta}{\partial \eta_{j, e}}\right)=0$

whose solution is zero, that is zero correction of the water levels. Moreover, since we adopt a fully-implicit time solution of the above system, any numerical instability will be dampened by the numerical diffusion.

Another property of the model is its capability to solve the wetting and drying problem without losing mass conservation. This is because the original continuity equation in the set of ODEs solved in each element along the prediction step is always saved. If water depth, in the circumcentre of the element, becomes zero or negative (from solution of the previous correction problem), momentum equations are changed according to specific approximation (see details in [6,7]), but the continuity equation is not. This gives also the possibility of propagating the front of the wave along several dry elements along a single time step. In the following linearized correction step, small negative water depths can be computed specially in the tail of the propagate waves, and the corresponding volumes are kept as negative both in the local and in the global mass balance. If, after the solution of the prediction step, water depth in element $e$ is zero or negative, the corresponding off-diagonal terms of the system matrix are set equals to zero (according to Eq. (42)) and zero fluxes $\tilde{F}_{j, e}$ are computed for element $e$ (see Eq. (43)).

\section{Numerical tests}

We present seven numerical tests. We compare results computed by the proposed algorithm with experimental data collected in lab flumes and results computed by other literature numerical schemes. Viscous terms are neglected in the governing PDEs system, except for the sixth test, where we investigate the capability of the proposed element ordering procedure (see Section 2) in flow fields with strong recirculation zones. Last test is finalized to study the convergence order of the proposed model according to a given exact solution. In some of the presented tests we show also the results computed by the previous MAST scheme proposed in [7], in order to investigate the improvements of the present proposed model. We investigate also the computational costs.

The computed local and global mass balance error for the following proposed tests is of the order of machine precision, approximately $1 . d-16$.

\subsection{Test 1. 2D dam-break experiment by Fraccarollo and Toro [18]}

The experimental flume has a $(2 \times 3) \mathrm{m}^{2}$ rectangular bottom plane, partially occupied by a reservoir, (see Fig. 5). The downstream part of the bottom plane is initially dry. Walls and bottom Manning coefficient is $0.0095 \mathrm{~s} / \mathrm{m}^{1 / 3}$. The width of the movable gate, symmetrically centred, is 0.40 wide $\mathrm{m}$. The flood-plain boundaries are all open. The Authors in paper [18] measured pressure, water depths and velocity components. Measure points are shown in Fig. 5 and their spatial coordinates can be found in [18].

Two sets of runs have been carried out, assuming horizontal bottom plane in the first run and 7\% bottom slope along $x$ direction in the second run.

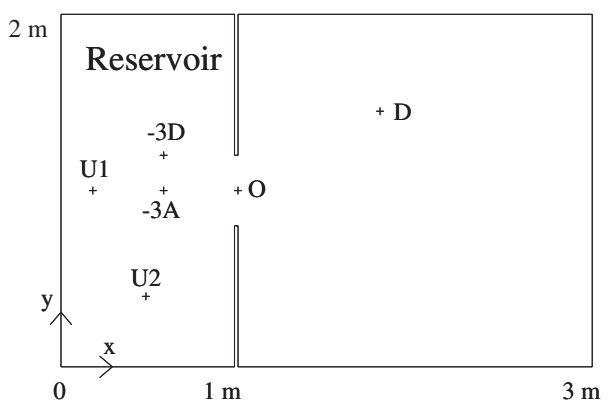

Fig. 5. Test 1. Lab flume geometry and position of the measure points.
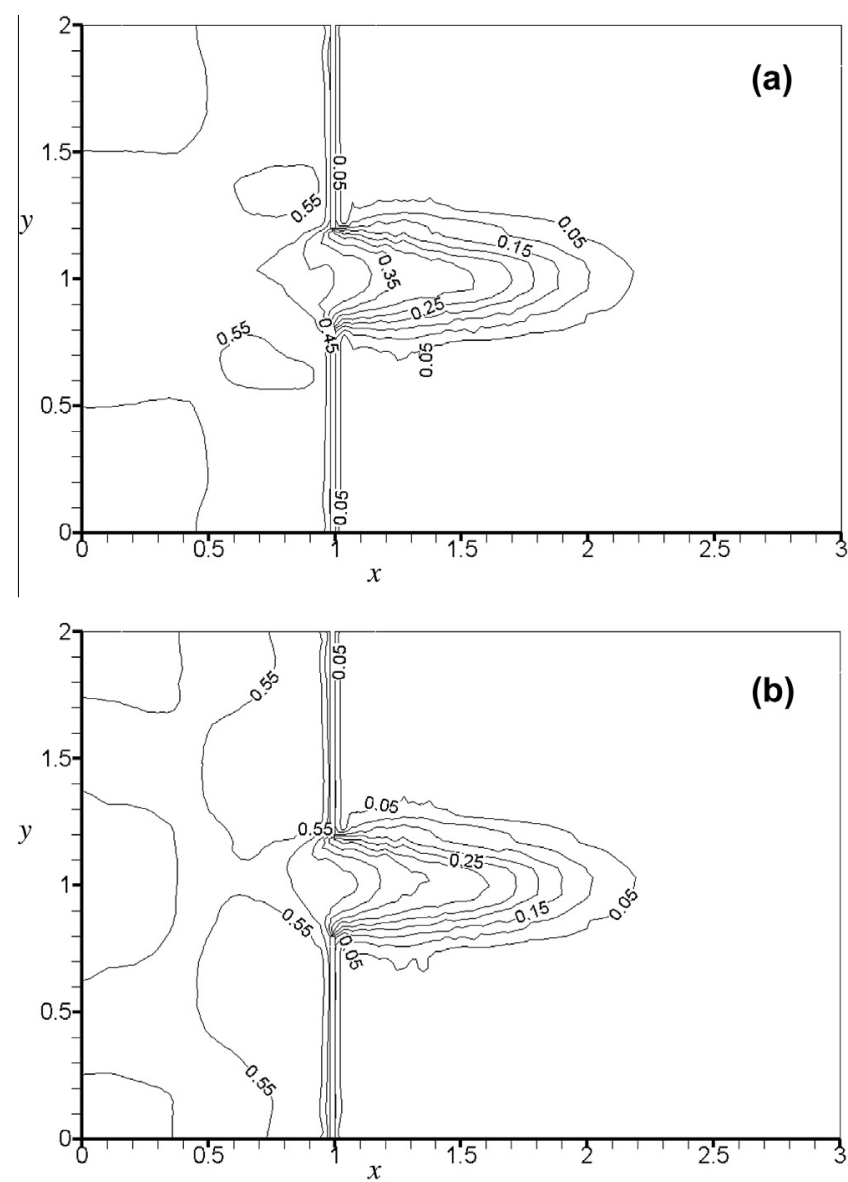

Fig. 6. Test 1 . Computed iso- $h$ contours at $t=0.5 \mathrm{~s}$. (a) zero bottom slope (b) 0.07 bottom slope along $x$ direction. 
Numerical results of the proposed model have been compared with the experimental data in [18] and with the numerical results by Fraccarollo and Toro [18] and Singh et al. [41]. In the two series of runs, the proposed scheme computes results very similar to the ones provided by the previous algorithm [7] and for brevity only the new data are shown.

Fraccarollo and Toro [18] applied a WAF (Weighted Averaged Flux) scheme, a 2nd order conservative, shock-capturing FV Godunov-type scheme. The Authors discretized the domain with a regular mesh of $150 \times 50$ points along $x$ and $y$ directions respectively. Singh et al. [41] applied a well balanced FV Godunov type scheme. They discretized the domain with a regular quadrilateral mesh with side $0.01 \mathrm{~m}$ and used a time step size which maintained the maximum CFL number less than 0.25 [41].

For the present model simulations, spatial domain is discretized with a GD triangulation of 8650 triangles and 4492 nodes. A time step $\Delta t=0.01 \mathrm{~s}$ has been used. The final mesh has been obtained from the one used for the simulation in [7] after the edge swap procedure mentioned in Section 5 and explained in [9].

In the experiment with zero bottom slope, the initial water depth inside the reservoir is $0.6 \mathrm{~m}$. Fig. 6(a) shows contours of the iso- $h$ lines obtained at simulation time $t=0.5 \mathrm{~s}$. The maximum CFL number for MAST scheme is 3.14. The asymmetric contours, specially for the smaller water depths, is due to the mesh asymmetry. In Fig. 7(a)-(d) we compare the measured and the computed water depths at points "U1", "U2", "O" and "D". All the numerical models are in good agreement with the experimental data at points "U1", "U2" and "D". Observe the difference between measured and numerical results for small simulation times at point "O", where the shallow water hypothesis does not hold. After the sudden opening of the gate a strong rarefaction wave starts moving in upstream direction. As in the 1D case, water depth at the gate location (point "O" in the specific case) drops to a local minimum value (about 4/9 of the initial depth) after the opening of the gate. This is analogous to the 1D exact solution in an horizontal and frictionless channel (see [37] and cited references). After the minimum water depth is reached, a rising stage follows, due to the fluxes coming from the wall boundaries and to 2D effects.

The delay between measured and computed data at point " $O$ " is likely due to the small time required for the real opening of the gate (about $0.1 \mathrm{~s}$ ).

Inside the reservoir, Fraccarollo and Toro [18] measured also the static pressure values at the bottom. Pressure measures are reported in meters of water column. For measurement points "U1" and "U2" pressures and water levels values are very close to each other and only water levels are reported in Fig. 7(a) and (b); at point " $O$ ", as expected, measured levels and hydrostatic pressures do not match because of the vertical velocity components.

In Fig. 8(a)-(d) the computed mean velocity components are compared with the corresponding measured ones at points "$3 \mathrm{D}$ ", "O" and "-3A". Along the vertical of each experimental point, velocity components have been measured at 8 levels with increasing distances from the bottom, starting from 0.05 up to $0.4 \mathrm{~m}$ and then averaged. Figures show also results by Fraccarollo and Toro [18]. Observe that both algorithms provide results substantially different from the measured values, specially for the shortest times from the dam break (i.e. $t \leqslant 3-\mathrm{s}$ ). At point " $\mathrm{O}$ ", measured velocity decreases almost monotonically after its maximum value; on the opposite, at points "-3A" and "-3D" measured velocity shows an oscillating behaviour. MAST model reproduces these trends, even though at point "O", for $t<0.05 \mathrm{~s}$, high frequency dampening oscillations appear. The oscillations at points "-3A" and "-3D" are more irregular than the measured ones. Moreover, observe that the amplitude of the oscillations at the first duration is even $50 \%$ more
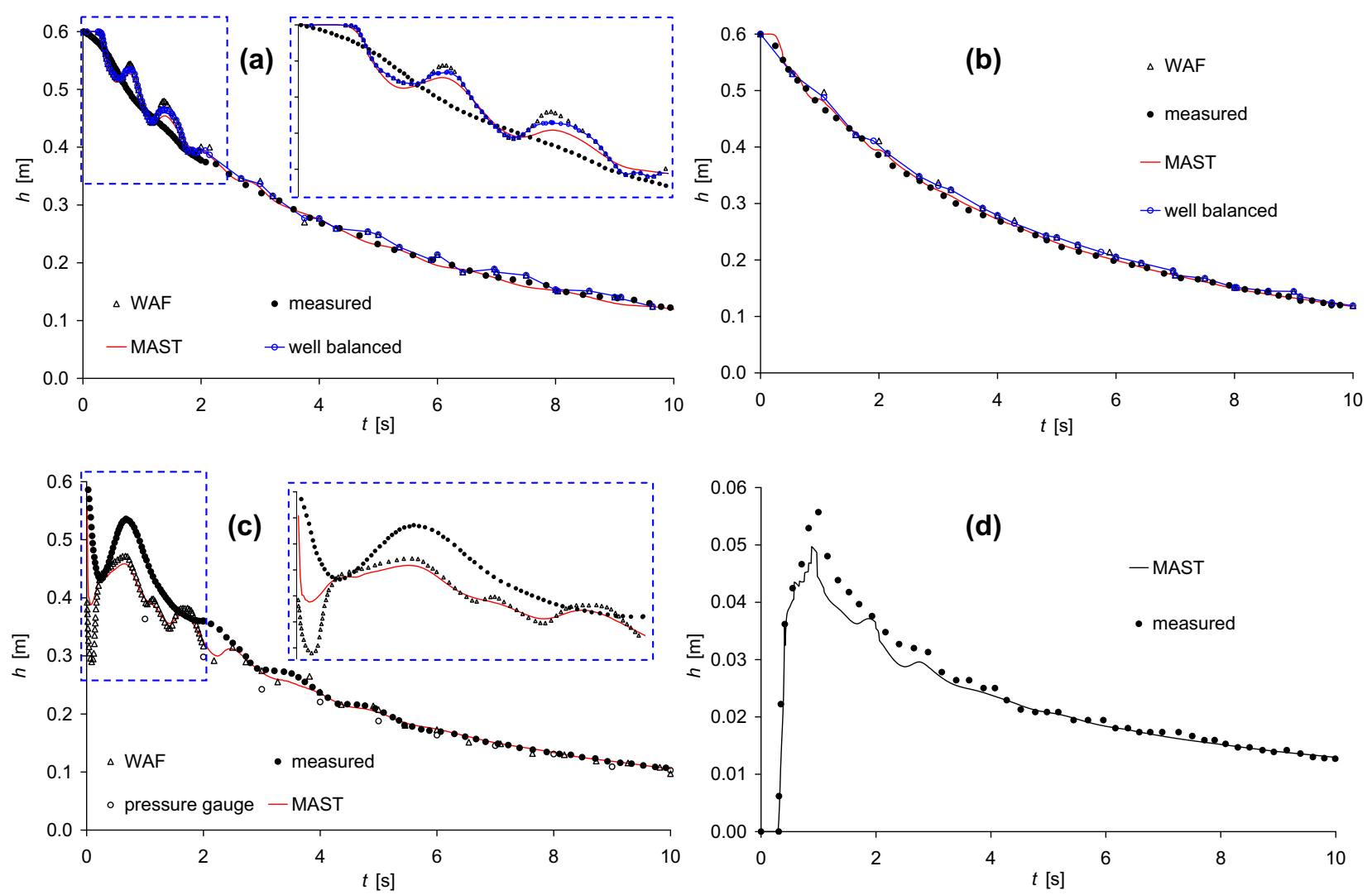

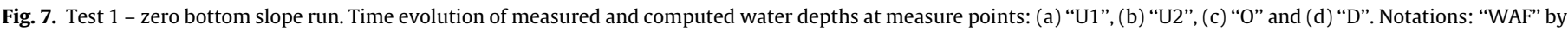
Fraccarollo and Toro [18], "measured" by Fraccarollo and Toro [18], "well balanced" by Singh et al. [41]. 

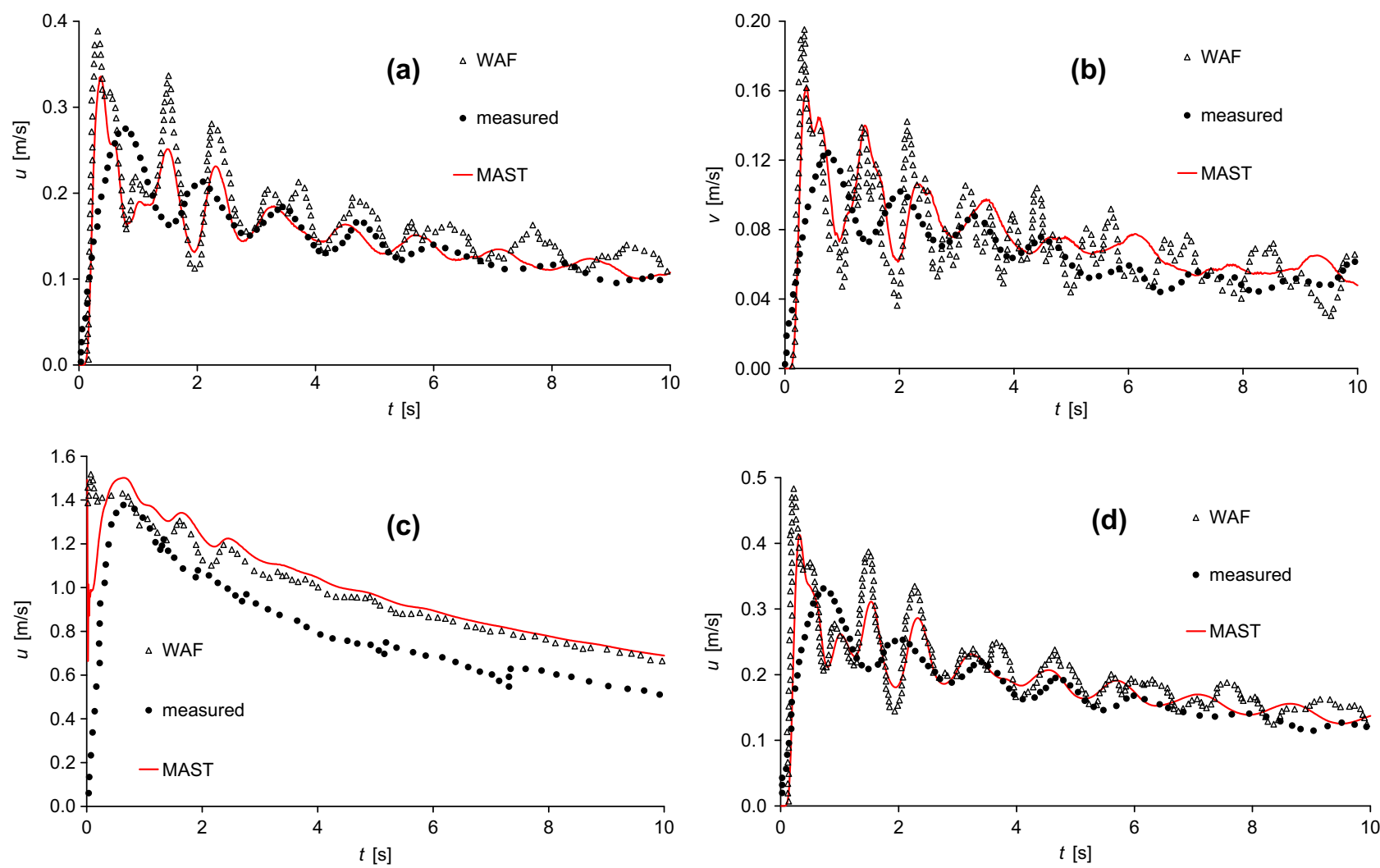

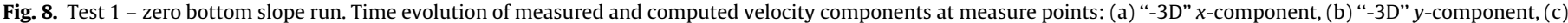
"O" $x$-component and (d) "-3A" $x$-component. Notations: "WAF" by Fraccarollo and Toro [18], "measured" by Fraccarollo and Toro [18].

than the measured value (see for example the $y$ velocity component at point "-3D"). The relative errors of the MAST computed velocities with respect to the measured ones are smaller than the corresponding ones provided by the WAF scheme, but are much larger than the relative errors of the computed water depths. A first reason is that the original unknowns of the model are the specific flow rate components and the water depth, instead of the velocity components. A second reason could be found in the measurement of the transient vertically averaged velocities, that is affected by a large uncertainty.

In the second set of runs the initial water depth value, measured at the wall foundation, is $0.64 \mathrm{~m}$.

Fig. 6(b) shows the computed MAST iso- $h$ contour lines at the simulation time $t=0.5 \mathrm{~s}$. Time step size $\Delta t$ is $0.01 \mathrm{~s}$ and the corresponding maximum CFL number is 4.22 . Observe also in this case

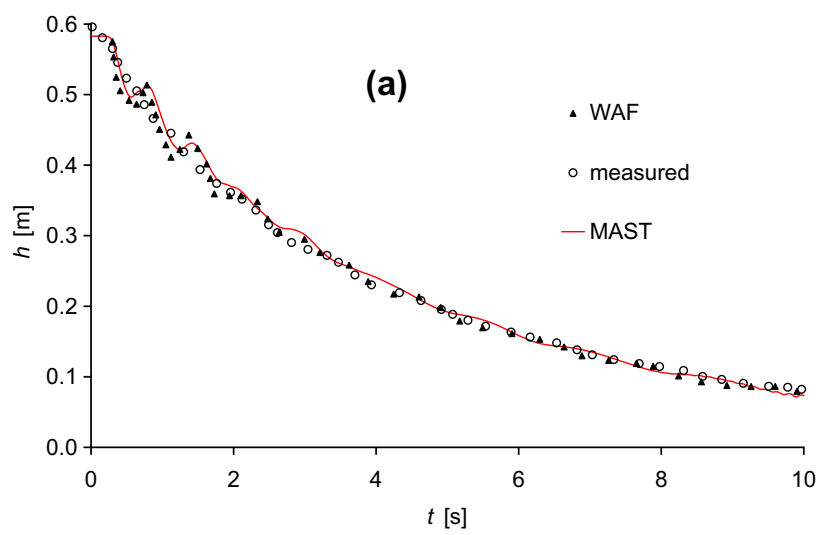

the asymmetry of the MAST results, similar to the zero bottom slope run.

In Fig. 9(a) and (b) computed water depths at points "U1" and "O" are compared with the corresponding measured values, as well as with the results by Fraccarollo and Toro [18] up to the simulation time $t=10 \mathrm{~s}$.

The proposed numerical scheme computes some circulations in the flow field during the simulation, due to the spatial discretization as discussed in Section 2. The following numerical experiment has been carried out. The above GD mesh ( 8650 triangles) has been refined three times as described in Section 2. Time step size has been halved at each refinement level, in order to limit the growth of the CFL number. Fluxes along the flow field circulations have been computed for each iteration and their values decrease dramatically refining the mesh. Similarly to the example shown in

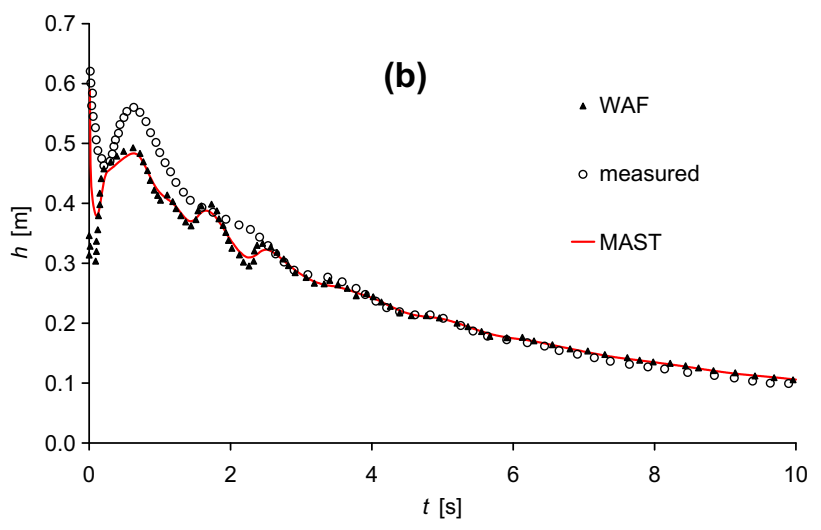

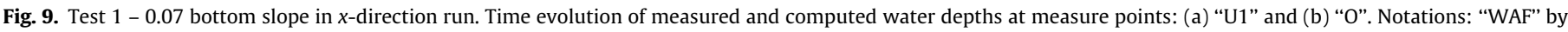
Fraccarollo and Toro [18], "measured" by Fraccarollo and Toro [18]. 
Section 2, the mean value of the circulation fluxes computed along a closed path of the flow field is $4.34 \mathrm{~d}-06 \mathrm{~m}^{3} / \mathrm{s}$, but reduces to $3.28 \mathrm{~d}-10 \mathrm{~m}^{3} / \mathrm{s}$ refining the mesh.

\subsection{Test 2. 2D Dam-break experiment in a L-shaped channel [43]}

Two sets of experiments have been carried out in the Civil Engineering Laboratory of the Catholic University of Louvain (Belgium) in a L-shaped channel form and rectangular cross section (Fig. 10) [43]. Bottom slope is zero in both $x$ and $y$ directions.

The upstream reservoir is a tank with rectangular $(2.44 \times 2.39) \mathrm{m}^{2}$ planar section, closed with a vertically sliding gate. The bottom level of the channel is $0.33 \mathrm{~m}$ higher than the reservoir bottom level, with a vertical step at the channel inlet (Fig. 10). In the experiments, the gate is pulled up very quickly and the closure failure is assumed as instantaneous. The channel is equipped with a set of gauges and their location can be found in the paper [43]. The $n$ Manning friction coefficient is $0.0095 \mathrm{~s} /$ $\mathrm{m}^{1 / 3}$ and the wall friction effect has been neglected [43].

The initial water level in the upstream reservoir measured from the channel bottom level is $0.2 \mathrm{~m}$ and the corresponding water depth, measured from the bottom reservoir, is $0.53 \mathrm{~m}$. The downstream channel is dry in a first set of runs and wet with a water depth $0.01 \mathrm{~m}$ in a second one.

When the gate is opened, the water flows rapidly into the channel and reaches the bend after approximately $3 \mathrm{~s}$. There, the water reflects against the wall, a bore forms and begins to travel in the upstream direction, back to the reservoir. For the water flowing downstream after the bend, multiple reflections on the walls can be observed.

A GD mesh with 10919 triangles and 5734 nodes has been used for the simulation of MAST algorithm. Time step $\Delta t$ is $0.01 \mathrm{~s}$.

Fig. 11(a) and (b) show the MAST computed iso- $h$ contour lines at the simulation times $3 \mathrm{~s}$ and $6 \mathrm{~s}$ and for the dry bed experiment. Model reproduces multiple reflections in the channel downstream the bend and no numerical oscillations of the water level occur in the transition reservoir-channel. Figs. 12(a)-(c) and 13(a)-(c) show the measured and computed water depth at some of the measure gauges for the two sets of experimental runs. Maximum CFL numbers computed by the present algorithm are respectively 1.71 and 2.26. In the same figures we show also results computed in both initially dry and wet bed conditions by Zhou et al. [51] using the SGMS algorithm, by Zhang et al. [49] using a CE/SE scheme, as well as, in dry bed conditions only, by Gottardi and Venutelli [19]. Gottardi and Venutelli [19] applied an explicit 2nd order central scheme, initially proposed by Kurganov and Tadmor [29]; integra-

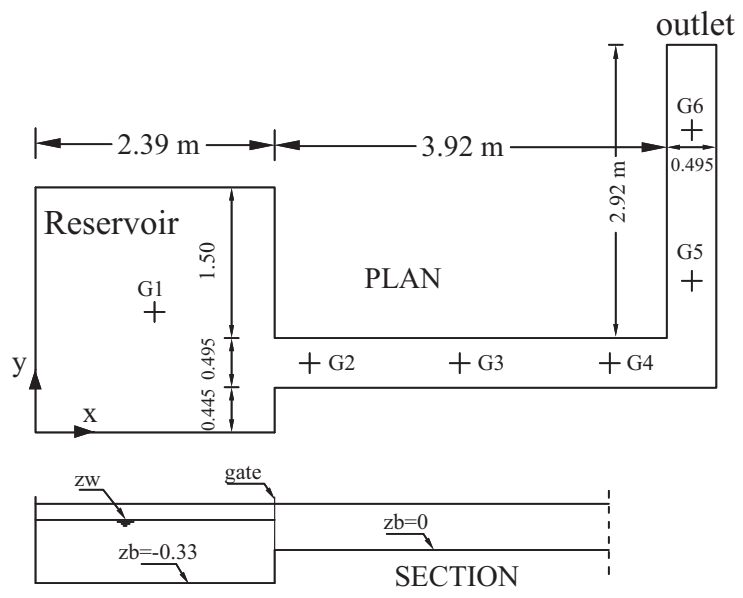

Fig. 10. Test 2. Lab flume geometry and position of the measure points.
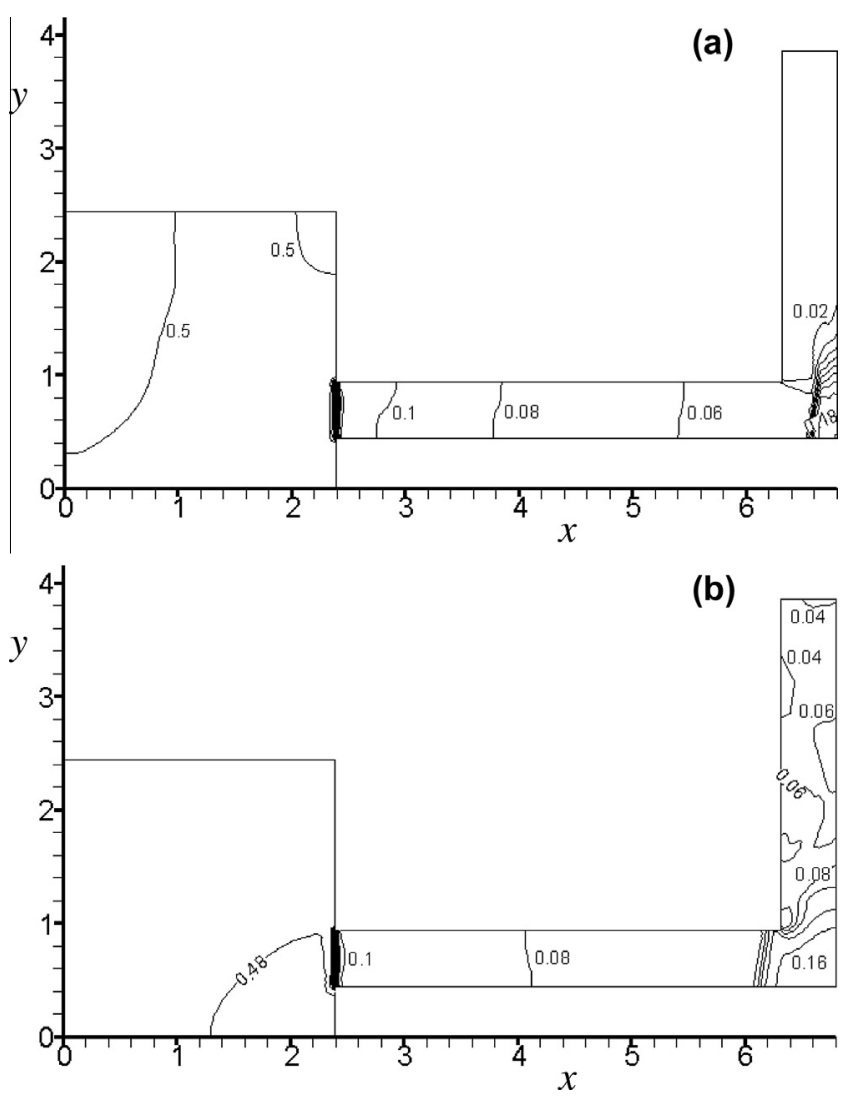

Fig. 11. Test 2 - dry-bed run. Computed iso- $h$ contours at: (a) $t=3 \mathrm{~s}$ (b) $t=6 \mathrm{~s}$.

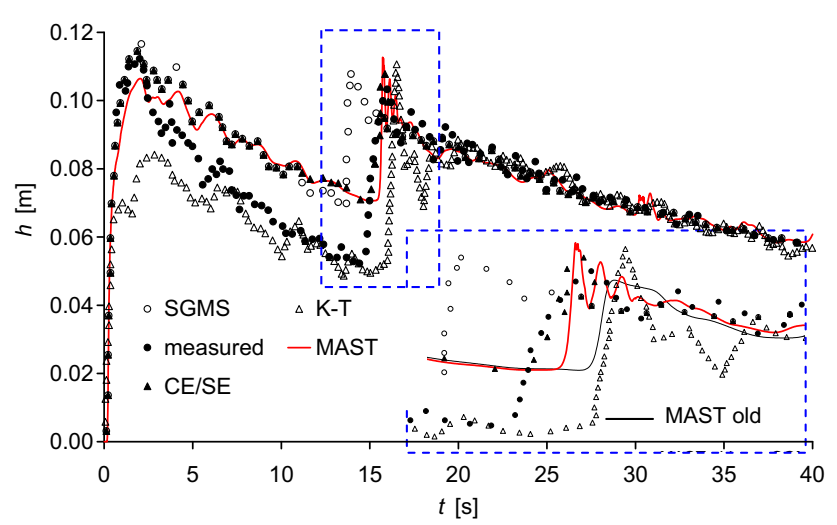

Fig. 12a. Test 2 - dry-bed run. Time evolution of the measured and computed water depth at gauge "G2". Notations: "measured" by Soares Frazão et al. [43], "MAST old" by Aricò et al. [7], "SGMS" by Zhou et al. [51], "K-T" by Gottardi and Venutelli [19] and "CE/SE" by Zhang et al. [49].

tion in time has been performed by means of a 3rd order TVD-Runge-Kutta scheme. Results by Gottardi and Venutelli [19] are marked as "K-T" in the following graphics. Zhou et al. [51] used a regular quadrilateral mesh with $\Delta x=0.05017 \mathrm{~m}$ and $\Delta y=$ $0.495 \mathrm{~m}$. Authors in [49] and [19] used squared quadrilateral meshes, with sides respectively 0.05 and $0.01 \mathrm{~m}$.

At gauge G1 inside the reservoir, all the numerical schemes compute very similar results in both wet and dry conditions. MAST results are very close to the ones computed in paper [7] and for brevity we refer the reader directly to paper [7].

Observe the time delay of the shock of the reflected wave in both MAST and Gottardi and Venutelli's [19] results with respect to the measured data at gauges G2, G3 and G4 and at gauges G3 


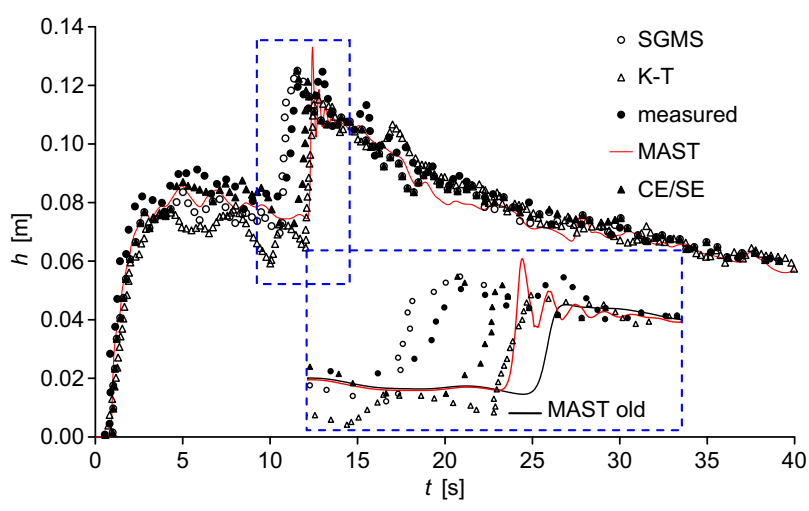

Fig. 12b. Test 2 - dry-bed run. Time evolution of the measured and computed water depth at gauge "G3". Notations: "measured" by Soares Frazão et al. [43], "MAST old" by Aricò et al. [7], "SGMS" by Zhou et al. [51], "K-T" by Gottardi and Venutelli [19] and "CE/SE" by Zhang et al. [49].

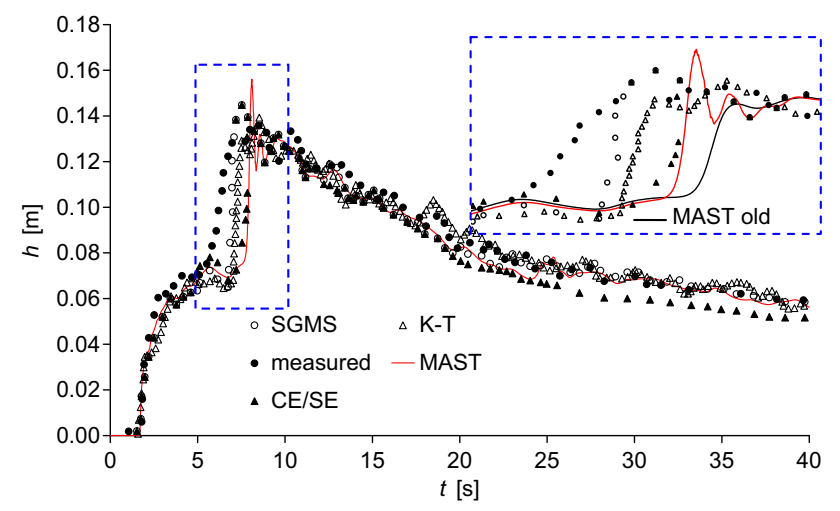

Fig. 12c. Test 2 - dry-bed run. Time evolution of the measured and computed water depth at gauge "G4". Notations: "measured" by Soares Frazão et al. [43], "MAST old" by Aricò et al. [7], "SGMS" by Zhou et al. [51], "K-T" by Gottardi and Venutelli [19] and "CE/SE" by Zhang et al. [49].

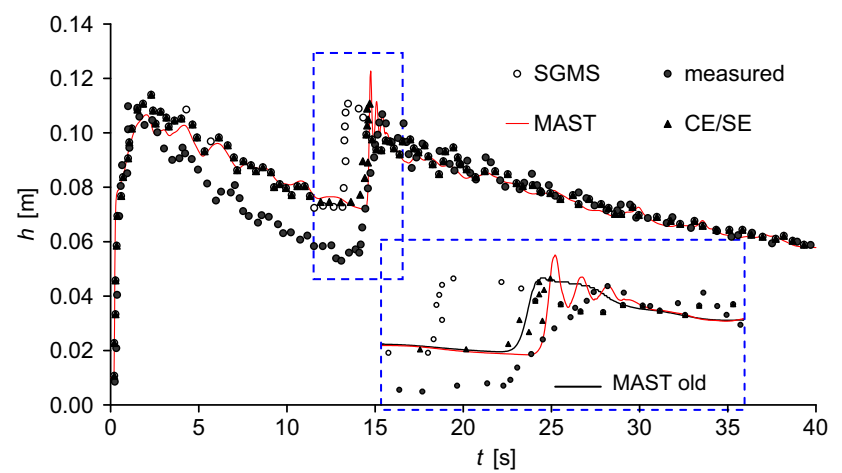

Fig. 13a. Test 2 - wet-bed run. Time evolution of the measured and computed water depth at gauge "G2". Notations: "measured" by Soares Frazão et al. [43], "MAST old" by Aricò et al. [7], "SGMS" by Zhou et al. [51], and "CE/SE" by Zhang et al. [49].

and G4 also for the CE/SE method. The delay of the MAST results is less than in the previous algorithm [7]. The MAST delay at gauge G4 is approximately $1.3 \mathrm{~s}$ in dry bed conditions and $1.05 \mathrm{~s}$ for wet bed conditions. The delay reduces progressively going from gauge G4 to gauge G2, where, in dry bed conditions, it is about $0.5 \mathrm{~s}$, while in wet bed conditions the new MAST algorithm reproduces very well the shock. In the previous algorithm [7] the effect of the reflected wave at gauge G2 in the numerical results arrives

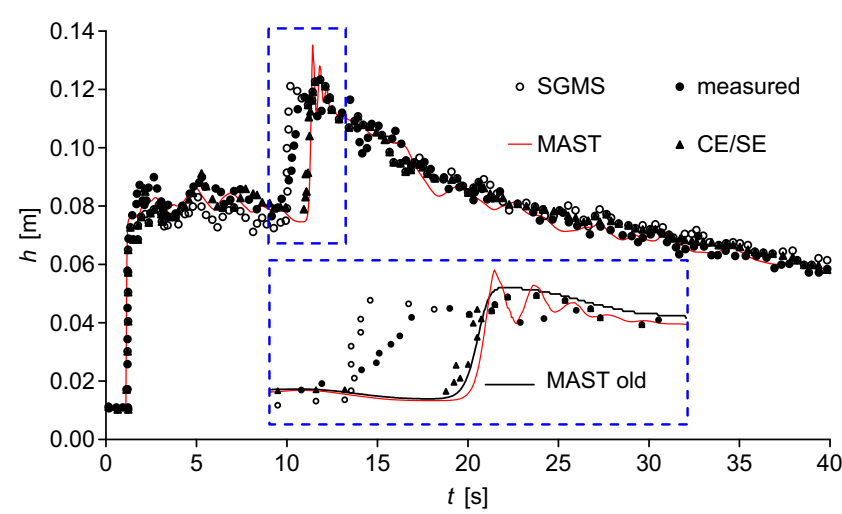

Fig. 13b. Test 2 - wet-bed run. Time evolution of the measured and computed water depth at gauge "G3". Notations: "measured" by Soares Frazão et al. [43] "MAST old" by Aricò et al. [7], "SGMS" by Zhou et al. [51], and "CE/SE" by Zhang et al. [49].

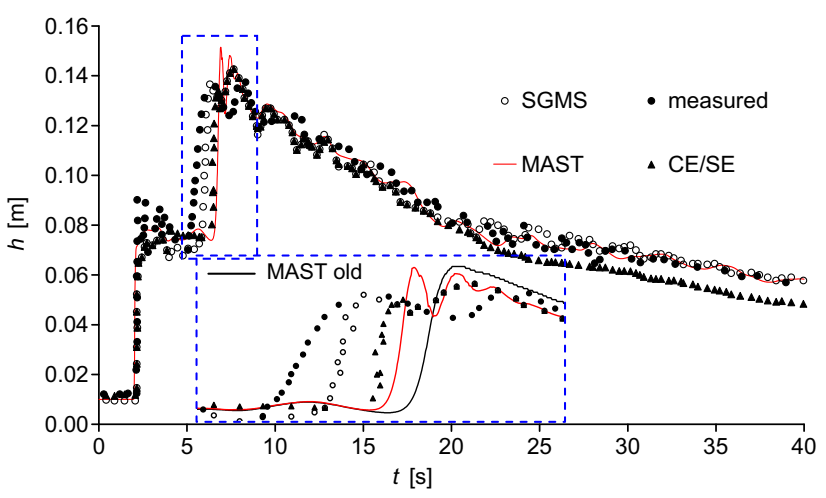

Fig. 13c. Test 2 - wet-bed run. Time evolution of the measured and computed water depth at gauge "G4". Notations: "measured" by Soares Frazão et al. [43] "MAST old" by Aricò et al. [7], "SGMS" by Zhou et al. [51], and "CE/SE" by Zhang et al. [49].
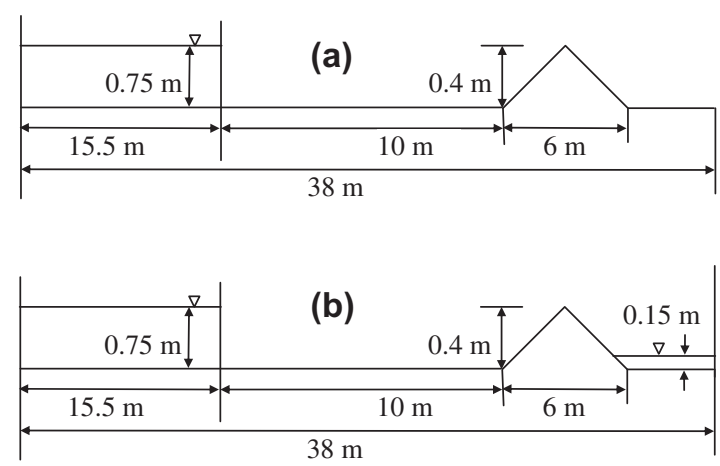

Fig. 14. Test 3. Lab flume geometry for: (a) free outflow downstream boundary condition ("scenario 1") and (b) high vertical wall downstream boundary condition ("scenario 2").

early (about $0.2 \mathrm{~s}$ ) with respect to the measured data. The CE/SE scheme [49] computes very well the shock wave at gauge G2, in both dry and wet runs, but it overestimates a lot water depth before the arrival of the shock and underestimates water depths after $20 \mathrm{~s}$. Results by Zhou et al. [51] are in good agreement with measured data at gauge $\mathrm{G} 3$, while the computed reflected wave shows a little delay (about $1 \mathrm{~s}$ ) at gauge G4 and an anticipation at gauge G2 (about $1.7 \mathrm{~s}$ and $1.45 \mathrm{~s}$ in dry and wet bed conditions). At gauge 
G2 both MAST and SGMS overestimate water depth before the arrival of the reflected wave and results are very similar. On the opposite, Gottardi and Venutelli [19] underestimate water depths approximately up to $10 \mathrm{~s}$.

At gauges G3 and G4, MAST scheme, as well as the models by Zhou et al. [51] and by Gottardi and Venutelli [19] provide similar result before and after the arrival of the reflected wave. After the arrival of the reflected wave, the three numerical schemes produce similar results also at the gauge G2. In the figures we show a zoom of the reflected shock wave where we plot also the results of the previous algorithm [7], marked as "MAST old".

Experimental data at points G5 and G6 are properly simulated by the MAST scheme in both wet and dry conditions. Since computed results are very close to the ones provided in paper [7], for brevity we refer the reader directly to [7].

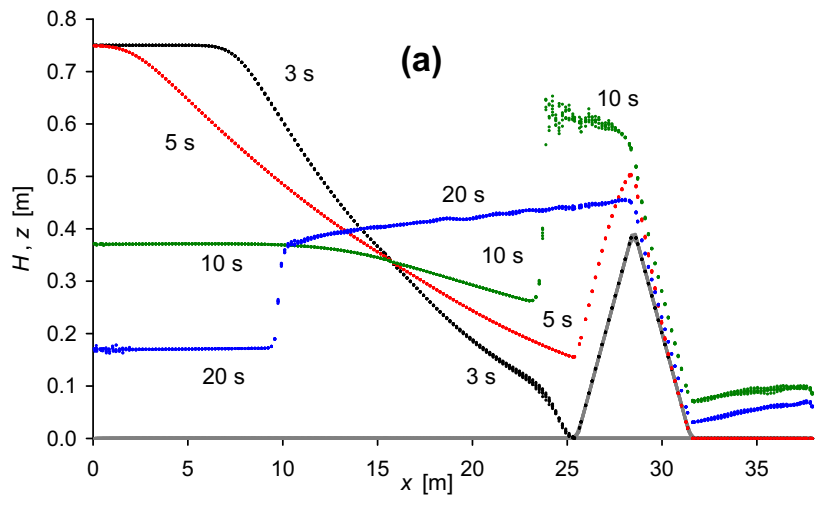

\subsection{Test 3. Experimental dam-break over triangular bump}

A dam-break experiment over a triangular bump has been carried out in the Laboratoire de Recherches Hydrauliques of the Universitè Libre de Bruxelles [12]. A reservoir $15.5 \mathrm{~m}$ long is filled up to $0.75 \mathrm{~m}$ level. A gate separates the reservoir from a dry, straight rectangular channel $22.5 \mathrm{~m}$ long, with a triangular bump $0.4 \mathrm{~m}$ high and $6 \mathrm{~m}$ long (see Fig. 14(a)). The channel has constant width $1.75 \mathrm{~m}$ and constant Manning friction coefficient, equal to $0.0125 \mathrm{~s} / \mathrm{m}^{1 / 3}$. Impervious boundary condition is given at the upstream end, where a solid wall is set, while two different downstream boundary conditions have been considered: free outflow (see Fig. 14(a)) and impervious boundary (see Fig. 14(b)). An initial water depth $0.15 \mathrm{~m}$ is assumed downstream the bump in the second case. In the following, the different runs for the two

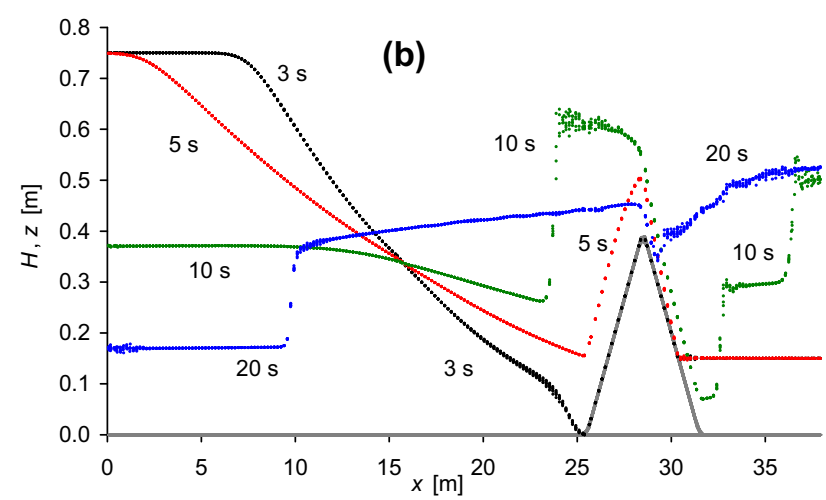

Fig. 15. Test 3. Computed water surfaces at different simulation times: (a) scenario 1; (b) scenario 2.
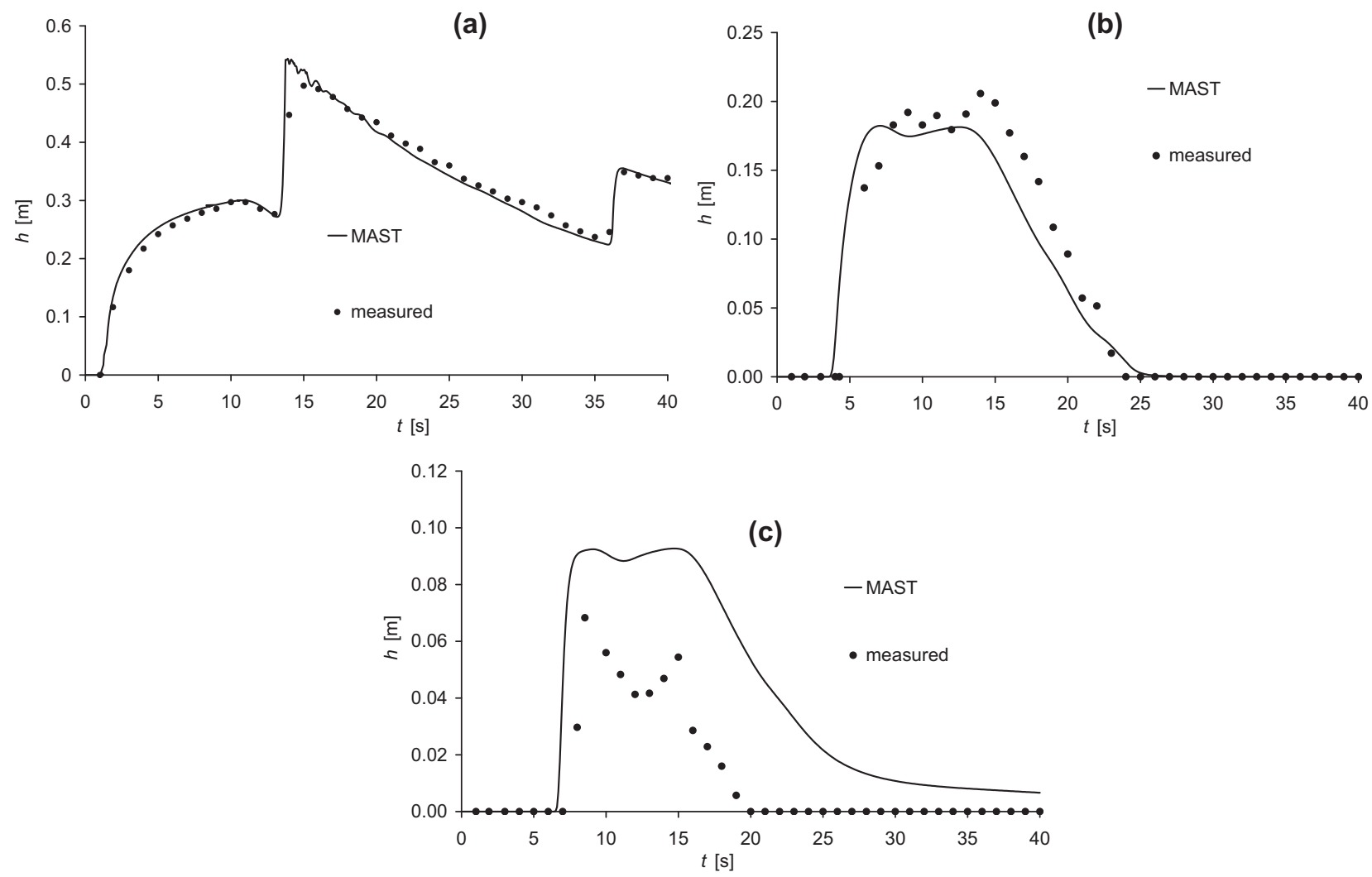

Fig. 16. Test 3 - scenario 1. Computed water depths at gauges: (a) "G4”, (b) "G13" and (c) “G20". Notations: "measured” by Brufau [12]. 
downstream boundary conditions will be marked as "scenario 1" and "scenario 2", respectively.

This case study has been presented in order to test the proposed model in multiple high-frequency wetting/drying processes. It is assumed that at $t=0$ the dam suddenly collapses and the initial still water in the reservoir rushes onto the downstream floodplain. After about $3 \mathrm{~s}$, the wet-dry front reaches the triangular obstacle and continues to climb over it. At around $t=5 \mathrm{~s}$, the front arrives at the other side of the horizontal floodplain. A shock wave forms due to the interaction between the incoming flow and the bed topography and starts to propagate in an opposite direction towards the upstream boundary. While the shock wave is travelling upstream, a rarefaction wave is developed and moves downstream, which causes the water depth above the bump to decrease. Multiple shock and rarefaction waves move in opposite directions along the channel, reflected either by the upstream wall, either by the bump, either, for the second experimental runs, by the water downstream the bump (see in [33] a qualitative description of the physical process). Wave interactions and wetting/drying processes continue until the flow momentum is damped by friction effects.

For the present model simulations, domain has been discretized with a GD triangulation with 1314 triangles and 773 nodes. Time step size is set to $0.1 \mathrm{~s}$. See in Fig. 15(a) and (b) the time evolution of the water depth profiles for the two scenarios. Profiles computed for scenario 1 are in very good agreement with the ones computed by Brufau et al. [13] (Authors in [13] provide results for the first scenario only). For simplicity, results by Brufau et al. [13] are not shown here. MAST model reproduces very well, without oscillations, the wave arriving at the base of the hump ( $t=3 \mathrm{~s}$ ), as well as the upstream travelling wave and the downstream travelling wave downstream the hump ( $t=5 \mathrm{~s}, 10 \mathrm{~s}$ and $20 \mathrm{~s}$ ).

Water depths have been measured at different experimental gauges, G2, G4, G8, G10, G11, G13 and G20, symmetrically located along the channel transverse direction, respectively at $2 \mathrm{~m}, 4 \mathrm{~m}$, $8 \mathrm{~m}, 10 \mathrm{~m}, 11 \mathrm{~m}, 13 \mathrm{~m}$ and $20 \mathrm{~m}$ downstream of the dam. Figs. 16(a)-(c) and 17(a)-(d) show the measured and the computed water depths for scenario 1. In Fig. 17(a)-(d) we compare the measured water depths with the ones computed by the presented model and by a well-balanced FV Godunov scheme by Liang and Marche [33]. This is a 2nd spatial approximation order, where non-negative reconstruction of Riemann states and compatible discretization of slope and friction source terms produce well-balanced solutions. Liang and Marche [33] used square meshes with

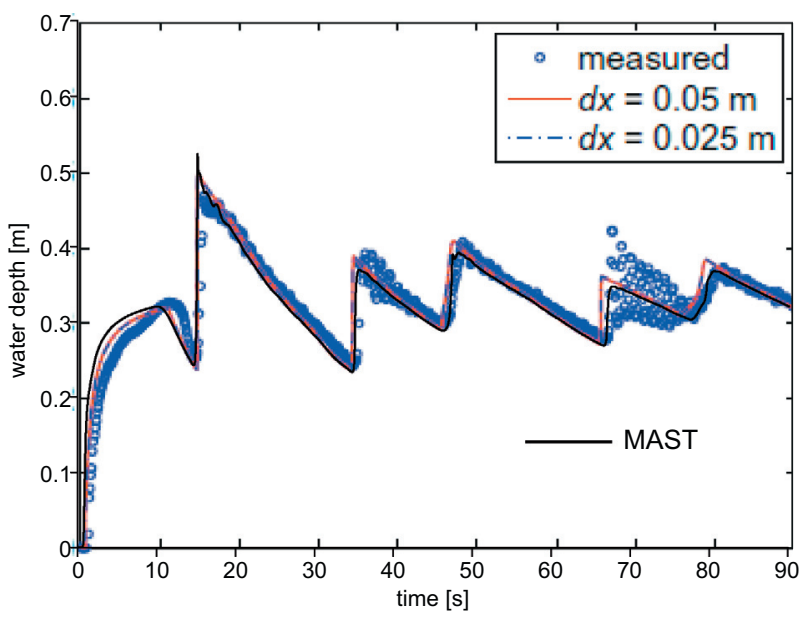

Fig. 17a. Test 3 - scenario 1. Computed water depths at gauge "G2". Comparison between the proposed scheme and the one by Liang and Marche [33]. Notations: "measured" by Brufau [12].

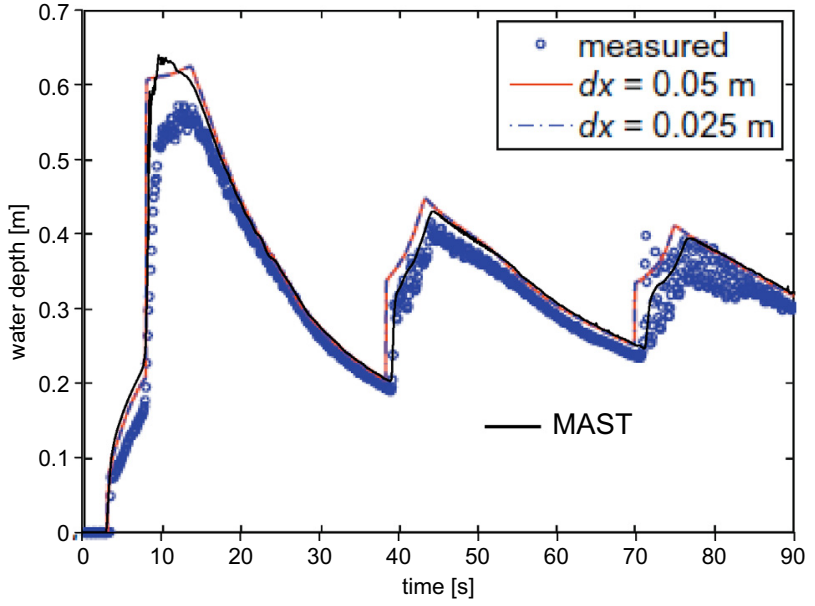

Fig. 17b. Test 3 - scenario 1. Computed water depths at gauge "G8”. Comparison between the proposed scheme and the one by Liang and Marche [33]. Notations: "measured" by Brufau [12].

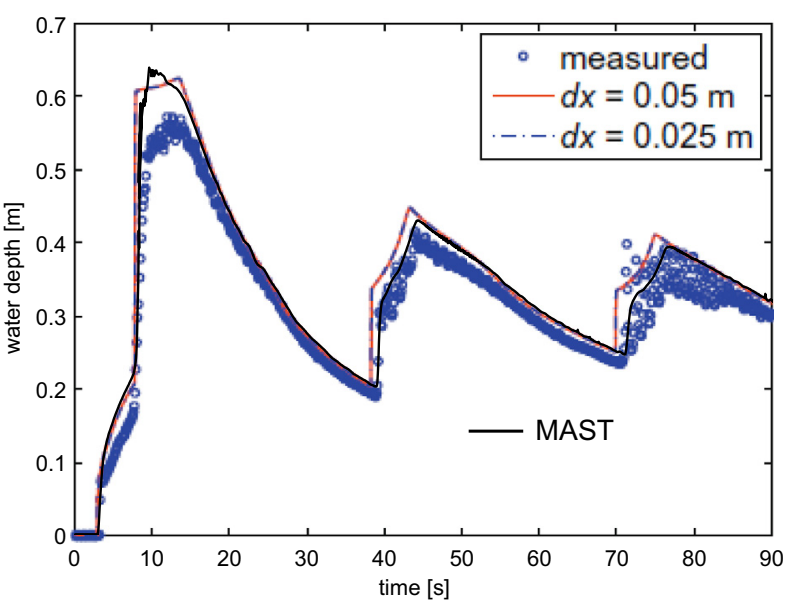

Fig. 17c. Test 3 - scenario 1. Computed water depths at gauge "G10". Comparison between the proposed scheme and the one by Liang and Marche [33]. Notations: "measured" by [12].

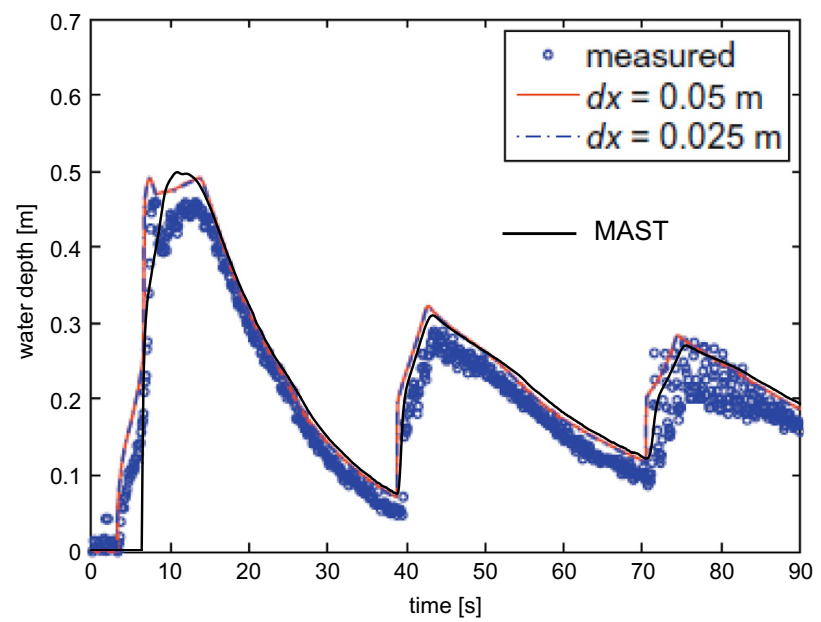

Fig. 17d. Test 3 - scenario 1. Computed water depths at gauge "G11". Comparison between the proposed scheme and the one by Liang and Marche [33]. Notations: "measured" by [12]. 
0.05 and $0.025 \mathrm{~m}$ side and time step size computed by the CFL criterion. They assumed a constant CFL number equal to 0.5 . Measured data are properly reproduced at gauges G2-G13 and overestimated at G20. At the gauging points located before the hump, the prediction of the arrival time of the wave as well as the water depth is good. Point G13 is located at the vertex of the obstacle and therefore is a critical point. It can be observed that prediction of the transitions from wet to dry is correct. Very similar results have been computed by Brufau et al. [13] and by Singh et al. [41] but are not shown here for brevity.

Fig. 18(a)-(d) show the experimental data and numerical results for scenario 2 at gauges G4, G10, G13 and G20. The numerical method overestimates and underestimates the water depths, respectively at points $\mathrm{G} 10$ and $\mathrm{G} 13$. At point $\mathrm{G} 4$ the proposed scheme reproduces properly the effect of the reflected wave. At gauge G20 MAST scheme overestimates a little bit the water depth. Maximum CFL values computed by MAST scheme are 2.35 and 2.12, respectively for scenario 1 and 2 .

\subsection{Test 4. Circular dam break problem}

This test case consists of the instantaneous breaking of a cylindrical tank (with diameter $20 \mathrm{~m}$ ) initially filled with $2 \mathrm{~m}$ of water at rest. The initial water depth outside the tank is $0.5 \mathrm{~m}$. When the column of water is released, wave generated by the breaking of the tank propagates into the still water with initial depth $0.5 \mathrm{~m}$ and the shock wave results in a significant increase of water depth in the lower depth region, propagating in the radial direction.

Domain is discretized using an unstructured mesh with 17942 triangles and 9154 nodes and the time step size is $0.05 \mathrm{~s}$. The maximum computed CFL value is 1.008 .

This test has been proposed in [14] and is useful to check the ability of the method to preserve cylindrical symmetry, since the problem becomes 1D in the radial direction.
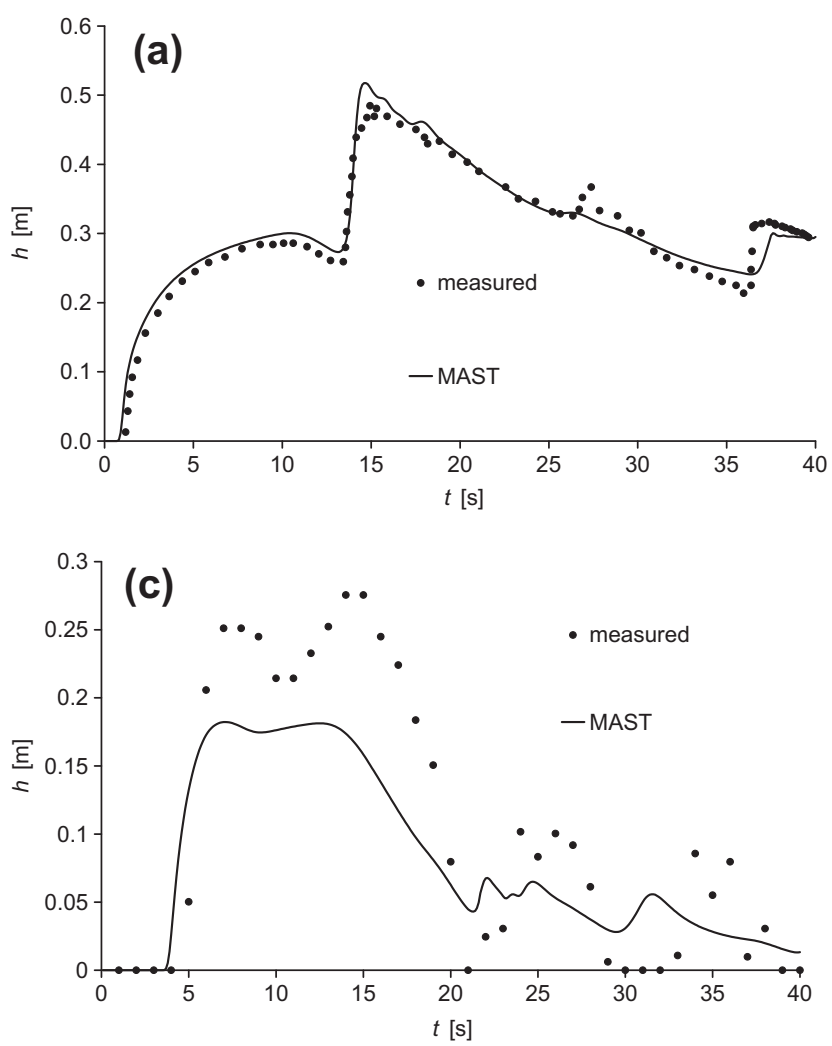

In Fig. 19(a)-(d) we show the iso - $h$ contours as well as the 3D view of the water depths at $t=1 \mathrm{~s}$ and $t=2.5 \mathrm{~s}$. In Fig. 20(a) and (b) we compare, for the same simulation times, the MAST results in radial direction (cut along a radial direction, which in this case corresponds to the $x$-axis) obtained over the above mesh with 17942 elements (marked as "MAST coarse mesh" in the figures) with the ones computed over a refined mesh, obtained from the first one dividing each side in two equal parts (marked as "MAST refined mesh" in the figures). As expected, the shock is captured in fewer elements using the refined mesh. In the same figures we show also the results obtained in [14]. The Authors in [14] proposed a well-balanced FV high-order centered scheme on unstructured meshes, PRICE2-C. They discretized the domain with 18050 triangles and fixed the CFL at 0.5 . In the same figures, a $1 \mathrm{D}$ reference numerical solution is shown too. This is presented in [14] and has been obtained solving the $1 \mathrm{D}$ equations written in a radial coordinate system, using a 1D version of the PRICE2-C model, the PRICE-C. More details can be found in [14] and cited references. According to fig. 19(a)-(d), MAST model properly reproduces the outward-propagating circular shock and the inward-propagating circular rarefaction wave. The model preserves cylindrical symmetry and does not compute unphysical oscillations. In Fig. 20 there is a good agreement between the MAST solution obtained over the coarse mesh and the one provided by the PRICE2-C as well as the 1D numerical reference solution.

\subsection{Test 5. Steady flow over a bump with shock}

A steady-state transcritical flow over a bump, with a smooth transition followed by a hydraulic jump is simulated. The channel is horizontal, frictionless, $25 \mathrm{~m}$ long and $1 \mathrm{~m}$ wide. Boundary conditions are given by:

$u h(0, t)=0.18 \mathrm{~m}^{3} / \mathrm{s} \quad h(L, t)=0.33 \mathrm{~m}$,
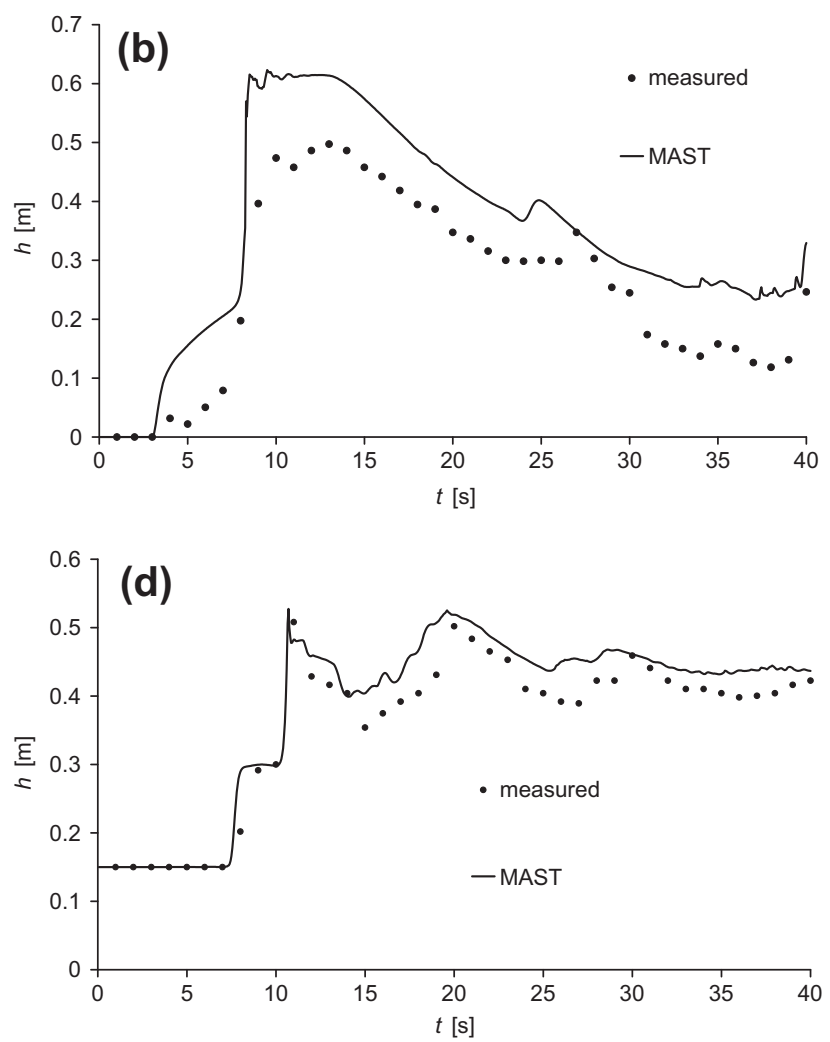

Fig. 18. Test 3 - scenario 2. Computed water depths at gauges: (a) "G4", (b) "G10", (c) "G13" and (d) "G20". Notations: "measured" by [12]. 
(a)

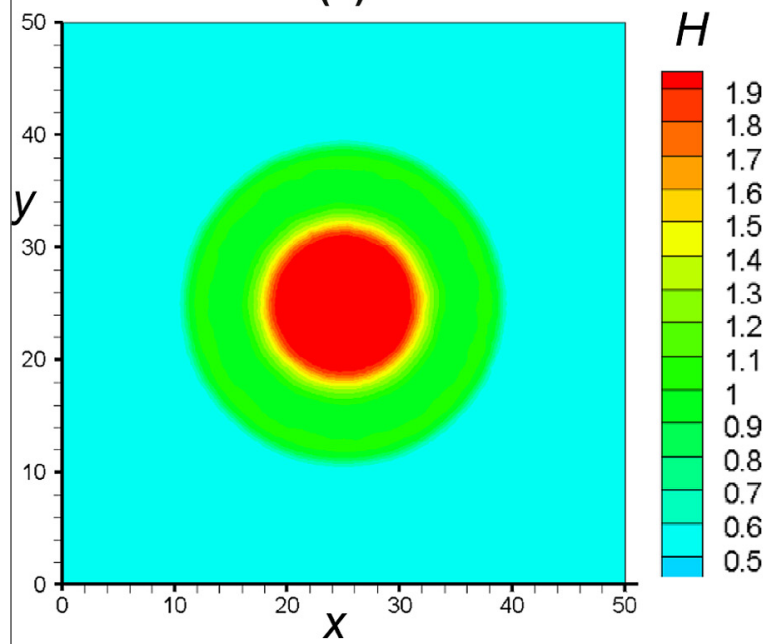

(c)

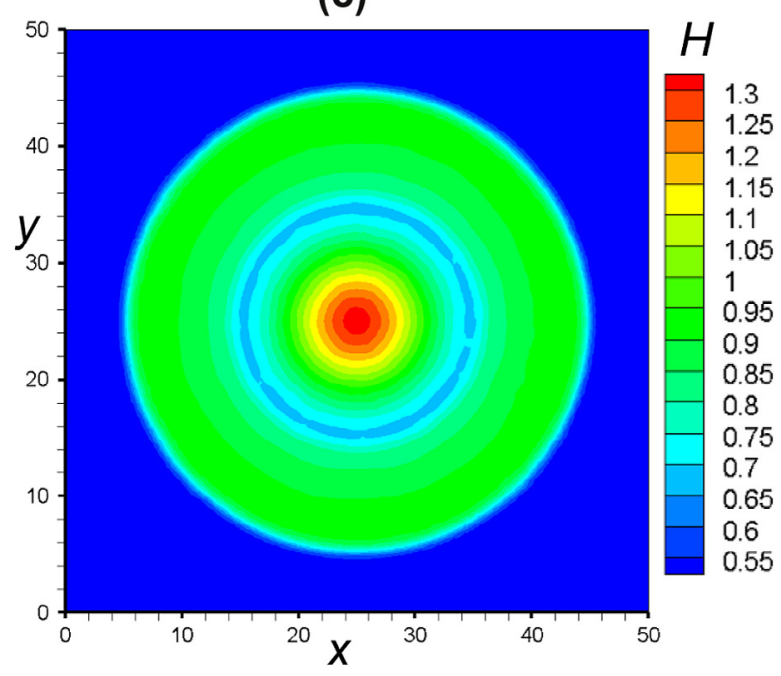

(b)
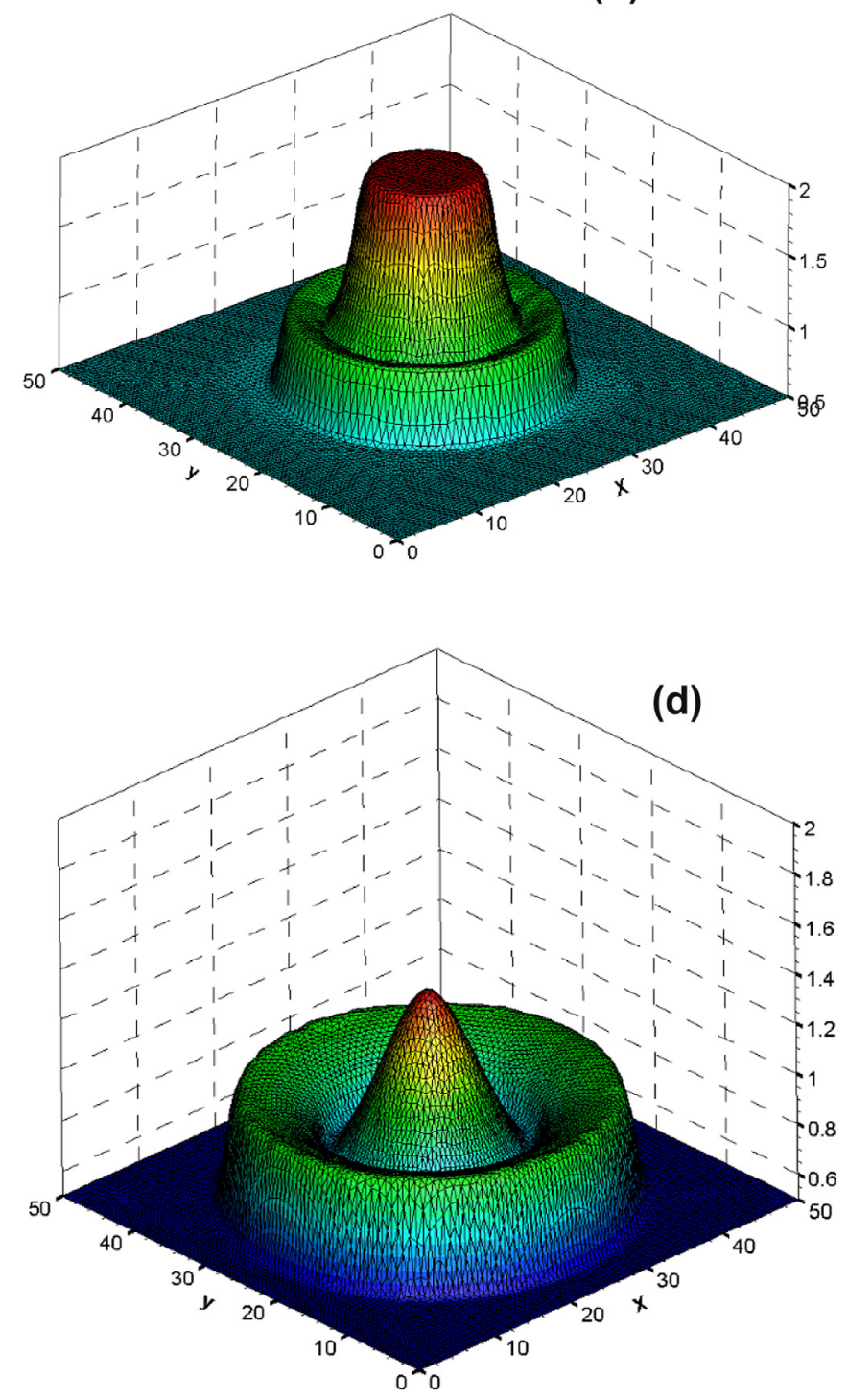

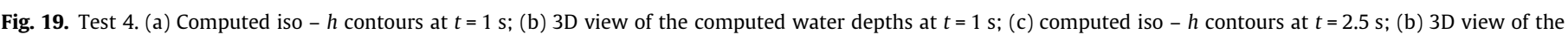
computed water depths at $t=2.5 \mathrm{~s}$.
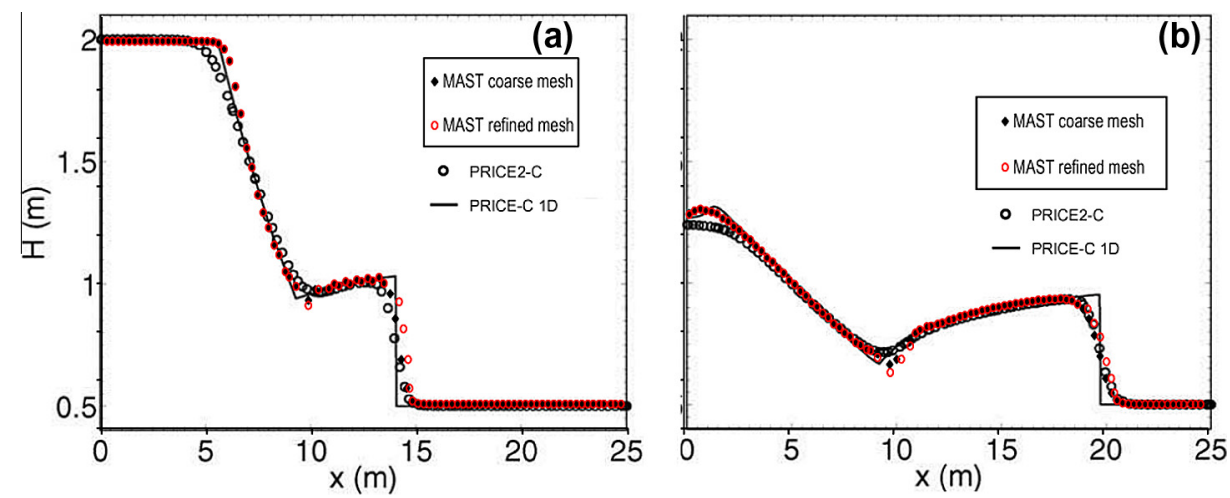

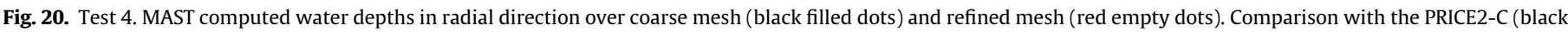

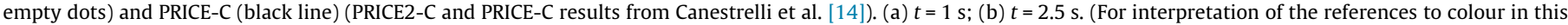
figure legend, the reader is referred to the web version of this article.) 
where $L$ marks the channel length. Initial condition is given by a constant water level over the channel, equal to $0.33 \mathrm{~m}$. Bed profile is given by:

$z(x)=\left\{\begin{array}{l}0.2-0.05(x-10)^{2} \quad \text { if } \quad 8 \leqslant x \leqslant 12 \\ 0 \quad \text { otherwise }\end{array}\right.$

This problem was proposed at the workshop on dam-break simulations [21] and essentially is a 1D test case often employed with 2D unstructured meshes to study the convergence to steady states in presence of discontinuities. For the MAST simulation, spatial domain is discretized using an unstructured GD mesh with 1503 triangles and 872 nodes. Time step size is $0.1 \mathrm{~s}$ and the maximum computed CFL number is 3.98. Fig. 21(a) and (b) show respectively the computed water levels and flow rates at simulation time $t=100 \mathrm{~s}$. Computed water levels are compared with the analytical solution provided in [21]. The hydraulic jump is captured in few elements and that no oscillations occur in the water surface and in the flow rate profiles along the bump. Increasing the iteration number, $u h$ value tends asymptotically to the stationary value $0.18 \mathrm{~m}^{3} / \mathrm{s}$, while $v h$ value is of order $1 \mathrm{~d}-14 \mathrm{~m}^{3} / \mathrm{s}$, in all the domain. Many papers report this numerical case, either for 1D either for 2D simulations. Most of the literature schemes provide accurate water levels simulation, but compute spurious oscillations in the flow rate profile (see for example [35] or [23]).

For the same test, we provide in Fig. 22 the convergence history of the water levels and module of the flow rate solutions. The global relative error $R$ is defined as [35]:

$R=\sqrt{\sum_{e=1, \mathrm{Nel}}\left(\frac{f_{e}^{n}-f_{e}^{n-1}}{f_{e}^{n}}\right)^{2}}$,

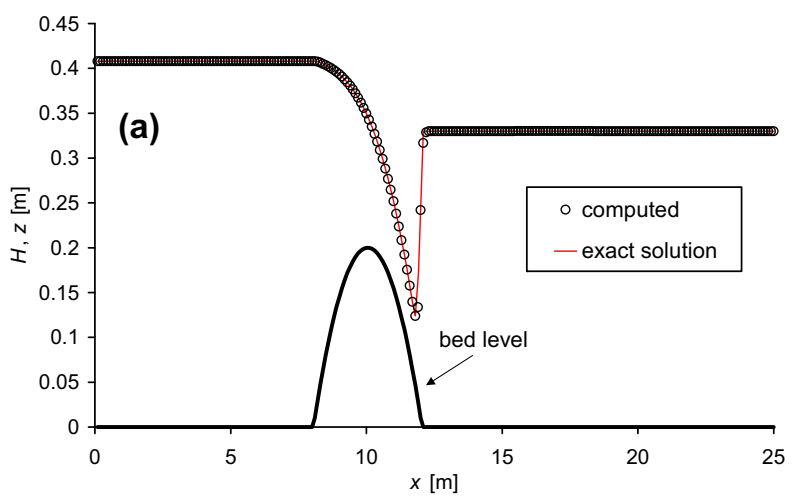

where $f_{e}^{n}$ is the water level or module of flow rate in the eth element computed at time level $n$th and index $n-1$ marks the corresponding variables computed at the previous time level.

\subsection{Test 6. Jet-forced flow in a circular basin}

A jet flow is forced in a shallow circular basin with a flat bed. This is a very useful test problem due to the complex geometry of the computational domain and has been proposed in several papers (e.g. $[4,38,47])$. Flow domain is bounded by straight-walled inlet and outlet stems connected to the curved-walled basin (see in Fig. 23 the channel geometry). The inlet into the reservoir is sharp-edged, and separation occurs giving rise to recirculation zones in both sides of the through-flow stream. The radius of the circular basin is $0.75 \mathrm{~m}$. Inlet velocity at the upstream end of the inflow stem is set to $0.1 \mathrm{~m} / \mathrm{s}$. The Authors of the above cited papers impose at the outlet of the downstream outflow stem a Dirichlet condition (water depth equal to $0.1 \mathrm{~m}$ ). Since the proper boundary condition depends on the Froude number computed at the downstream end, in the present work the downstream boundary condition is computed according to the model solution, as described in Section 4.4. The bed friction coefficient is zero and $v$ is $7.84 \mathrm{~d}$ $04 \mathrm{~m}^{2} / \mathrm{s}$. Spatial domain is discretized using two different GD triangulations: the first one is symmetric with respect to the centre of the circular basin, with 3112 triangles and 1695 nodes, the second one is non symmetric, with 3100 triangles and 1689 nodes. Time step size is $0.01 \mathrm{~s}$. Maximum computed CFL values are 1.089 and 1.07 respectively for the two meshes. Streamlines computed after $4.2 \mathrm{~s}$ and $7.5 \mathrm{~s}$ using the symmetric mesh are shown in Fig. 24(a) and (b), while Fig. 25(a) and (b) show the streamlines at the steady state, computed respectively over the symmetric and non

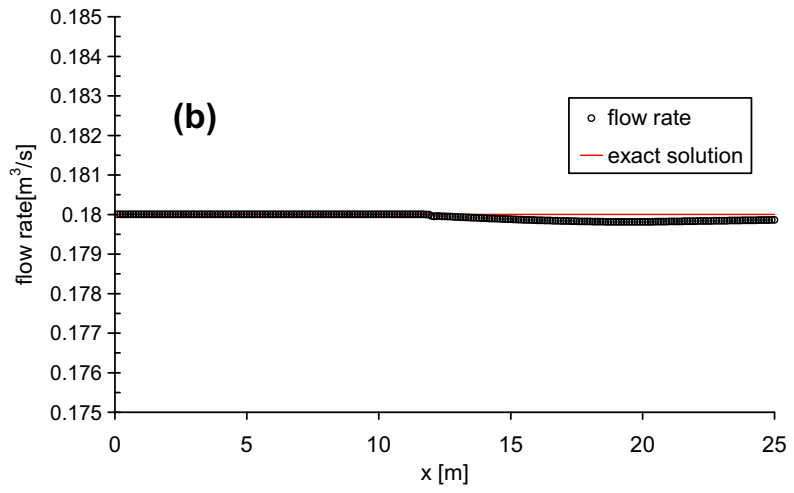

Fig. 21. Test 5. (a) Computed and exact (from Goutal and Maurel [21]) water levels at $t=100 \mathrm{~s}$. (b) Computed flow rates at $t=100 \mathrm{~s}$.

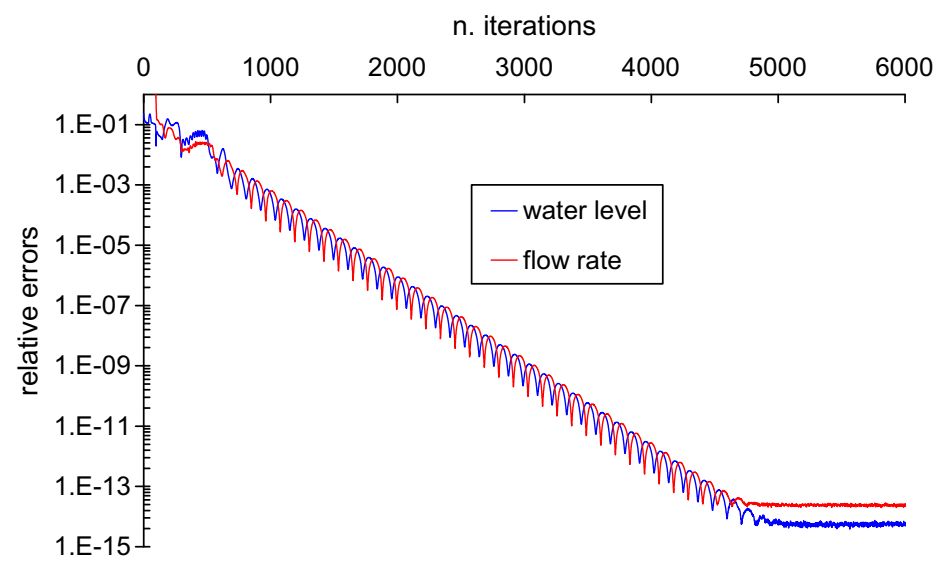

Fig. 22. Test 5. Convergence history of the water levels and module of the flow rate solutions. Relative error computed as in Eq. (65). 


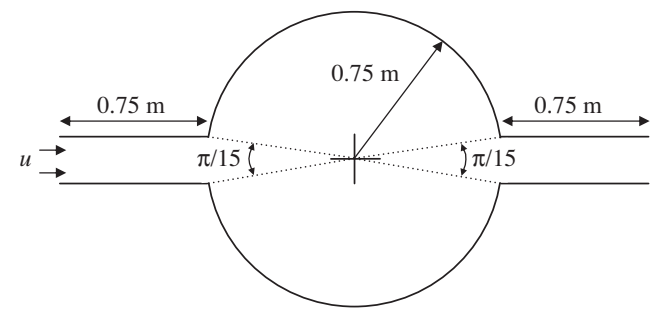

Fig. 23. Test 6. Lab flume geometry.
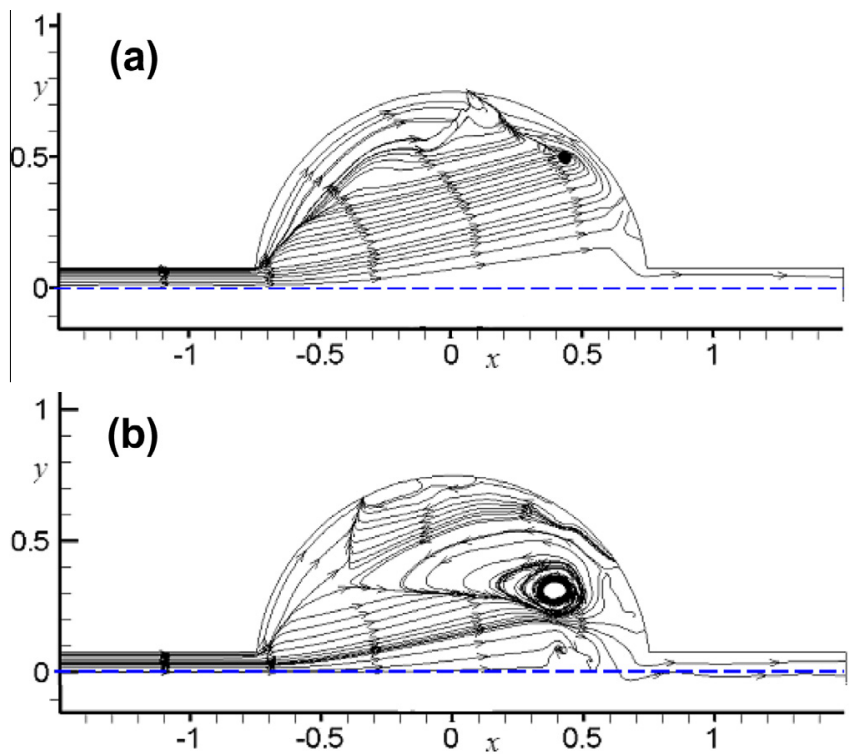

Fig. 24. Test 6 - symmetric mesh. Computed streamlines at: (a) $t=4.2 \mathrm{~s}$ (b) $t=7.5 \mathrm{~s}$.
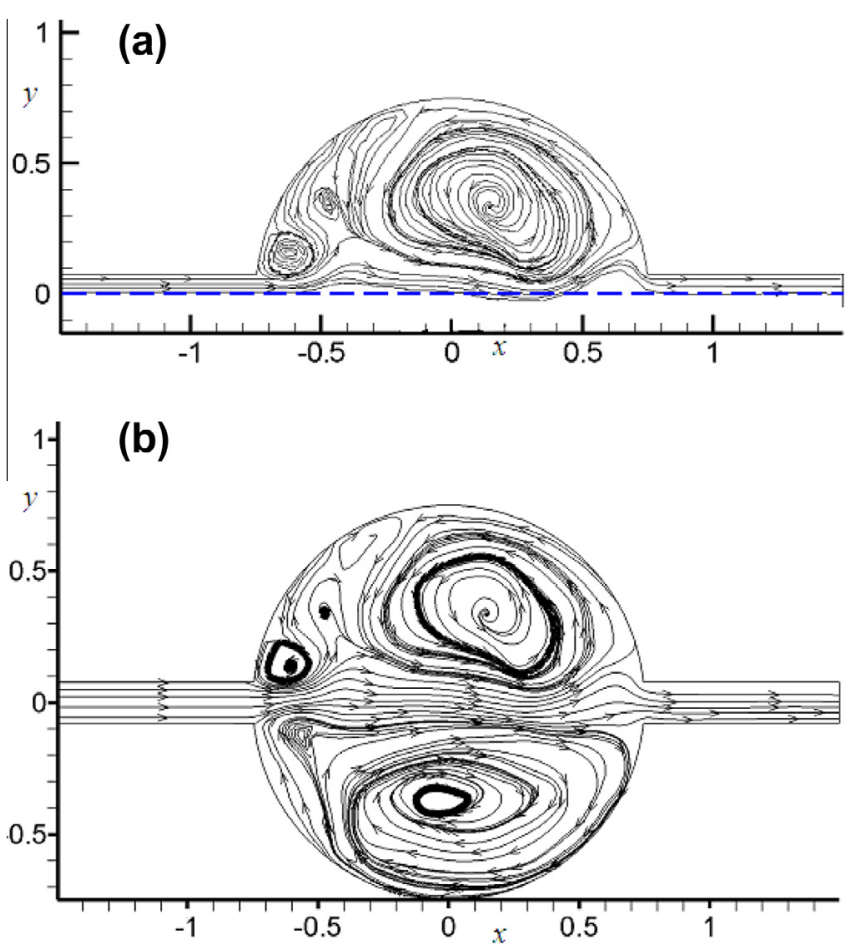

Fig. 25. Test 6 - Computed streamlines at steady state: (a) symmetric mesh (b) non symmetric mesh. symmetric mesh. The strong similarity of the steady state streamlines computed over the two meshes highlights the robustness of the model with respect to the mesh geometry. Several recirculation zones can be found in the flow field. Due to the spatial discretization error, the $O$ property is not satisfied and the procedure explained in Section 2 must be applied before each prediction step. See in Section 2 how the computed flux along a closed circuit in a particular area of the domain reduces by refining the computational mesh.

\subsection{Test 7. Moving shorelines in a 2D frictional parabolic bowl}

The analytical test of moving shorelines in a 2D frictional parabolic bowl was developed by Sampson et al. [40] and proposed by several Authors (see for example [23]). Computational domain is assumed squared with side $8000 \mathrm{~m}$ as in [23]. Bed topography, symmetrical with respect to the centre of the domain $\left(x_{0}, y_{0}\right)$, is given by Hou et al. [23]:

$z(x, y)=\frac{h_{0}\left(\left(x-x_{0}\right)^{2}+\left(y-y_{0}\right)^{2}\right)}{a}$,

with $x_{0}=y_{0}=4000 \mathrm{~m}$ and $h_{0}$ and $a$ two constants $\left(h_{0}=10 \mathrm{~m}\right.$ and $a=3000 \mathrm{~m}$ as in [23]). Authors in paper [23] write the friction source terms in the governing Eqs. (16) and (17) as:

$$
\begin{aligned}
& g n^{2} \frac{u h \sqrt{(u h)^{2}+(v h)^{2}}}{h^{7 / 3}}=C_{f} u \sqrt{u^{2}+v^{2}} \\
& g n^{2} \frac{v h \sqrt{(u h)^{2}+(v h)^{2}}}{h^{7 / 3}}=C_{f} v \sqrt{u^{2}+v^{2}} \\
& \text { with } C_{f}=\frac{g n^{2}}{h^{1 / 3}},
\end{aligned}
$$

and for the proposed test case they write roughness coefficients $C_{f}$ as: $C_{f}=\frac{h \tau}{\sqrt{u^{2}+v^{2}}}$,

with $\tau$ a constant value. According to Eqs. $(67, \mathrm{a})$ and $(67, \mathrm{~b})$ we get:

$\tau=\frac{g n^{2} \sqrt{(u h)^{2}+(v h)^{2}}}{h^{7 / 3}}$

The exact solutions for water level and velocity components are respectively $[40,23]$ :

$$
H(x, y, t)=h_{0}-\frac{B^{2} e^{-\tau t}}{2 g}-\frac{B e^{-\tau t / 2}}{g}\left[\begin{array}{l}
\left(\frac{\tau}{2} \sin s t+s \cos s t\right)\left(x-x_{0}\right)+ \\
\left(\frac{\tau}{2} \cos s t-s \sin s t\right)\left(y-y_{0}\right)
\end{array}\right],
$$

$u(t)=B e^{-\tau t / 2} \sin s t v(t)=B e^{-\tau t / 2} \cos s t$,

where $B$ is a constant given by the initial value of $v(t=0)$, $s=\sqrt{\left(p^{2}-\tau^{2}\right)} / 2, \quad p=\sqrt{8 g h_{0}} / a$. Authors in [23] assign $\tau=0.002 \mathrm{~s}^{-1}$ and $B=5 \mathrm{~m} / \mathrm{s}$. Initial conditions are obtained by the exact solution in Eq. (68) setting $t=0$. Starting from Eq. (67), formulation of the source terms of the prediction problem (in Eq. (21)) and of the elem coefficients of the correction problem (in Eq. $(42, a))$ changes accordingly as:

$$
\begin{aligned}
& R_{e}^{x}=\left|T_{e}\right|\left(g h_{e} \frac{\partial H_{e}^{k}}{\partial x}+\tau(u h)_{e}\right) \\
& R_{e}^{y}=\left|T_{e}\right|\left(g h_{e} \frac{\partial H_{e}^{k}}{\partial y}+\tau(v h)_{e}\right) \\
& \text { elem }_{e}=\frac{g \bar{h}_{e} \Delta t}{1+\tau \Delta t} .
\end{aligned}
$$

For the numerical simulation, domain has been discretized using an unstructured mesh with 272 elements and 149 nodes 
and the time step size is 40 . The total simulation time is $6000 \mathrm{~s}$ and the maximum CFL computed value is 2.8 . Computational mesh has been refined three times, as previously specified and the time step size has been halved to limit the growth of the CFL number. $L_{2}$ norms of the relative errors of the water depths $\left(L_{2, h}\right)$ and the specific flow rate components $u h$ and $v h\left(L_{2, u h}, L_{2, v h}\right)$, with respect to the exact values, have been computed. Results are reported in Table 1 . We assume the relative error computed for mesh level $l$, err proportional to a power of the linear size of the area of the mean triangle in the mesh,

$\operatorname{err}_{l}=\left(\sqrt{|T|_{l}}\right)^{r_{c}}$

where $|T|_{l}$ is the area of the mean triangle at refinement level $l$ and $\sqrt{|T|_{l}}$ represents a measure of its linear size. The rate of convergence $r_{c}$ is computed by comparing the relative errors of two successive refinement levels $l$ and $l+1$ :

$r_{c}=\frac{\log \left(\frac{e r r_{I}}{e r r_{l+1}}\right)}{\log (2)}$.

Table 1 shows the convergence order for water depth and specific flow rate components and these are close to 1 , as expected due to the spatial approximation order of the unknown variables assumed in the model, and remain almost the same refining the mesh. A constant convergence order along with the growth of mesh density is very important, because it implies stable results also when a coarse mesh is used instead of a very refined one. Fig. 26(a)-(d) show the computed and exact water levels and specific flow rates $u h$ and $v h$ in section D-D (the domain diagonal, bottom left corner - top right corner) at $t=500 \mathrm{~s}$ and $t=1500 \mathrm{~s}$. Results refer to the third refinement level mesh (17408 triangles and 8881 nodes). Computed results are in very good agreement with the exact ones. Moreover, model results are in very good agreement with the ones provided by Hou et al. [23], who proposed a 2nd spatial approximation order well-balanced FV Godunov-type scheme, equipped with WD treatment procedure. Hou et al. [23] discretized the domain with a Delaunay unstructured triangular mesh with a density similar to the third refined one used for the present model. For brevity we do not show results by Hou et al. [23] since, at the graphic scale, they are undistinguishable from the ones computed by the proposed model.

\subsection{Investigation of the computational costs (CPU times)}

Computational costs of the different algorithm steps have been investigated. Test 1 (in Section 5.1) with zero bottom slope has been selected for this analysis. Starting from the GD triangulation with 8650 triangles and 4492 nodes, three refinements have been

Table 1

Test 6. $L_{2}$ norms of relative errors of the water level and specific flow rate components and convergence order.

\begin{tabular}{|c|c|c|c|c|c|c|c|}
\hline Refinement level & $\mathrm{Nel}$ & $L_{2, h}$ & $r_{c, h}$ & $L_{2, u h}$ & $r_{c, u h}$ & $L_{2, \mathrm{v} h}$ & $r_{c, \mathrm{vh}}$ \\
\hline 0 & 272 & $4.67 \mathrm{E}-02$ & & $5.15 E+00$ & & 3.812874 & \\
\hline 1 & 1088 & $1.79 \mathrm{E}-02$ & $1.39 \mathrm{E}+00$ & $1.99 \mathrm{E}+00$ & $1.38 \mathrm{E}+00$ & 1.451446 & $1.39 \mathrm{E}+00$ \\
\hline 2 & 4352 & $7.17 \mathrm{E}-03$ & $1.32 \mathrm{E}+00$ & $8.38 \mathrm{E}-01$ & $1.24 \mathrm{E}+00$ & 0.619203 & $1.23 \mathrm{E}+00$ \\
\hline 3 & 17408 & $3.02 \mathrm{E}-03$ & $1.25 \mathrm{E}+00$ & $3.70 \mathrm{E}-01$ & $1.18 \mathrm{E}+00$ & 0.283403 & $1.13 \mathrm{E}+00$ \\
\hline
\end{tabular}
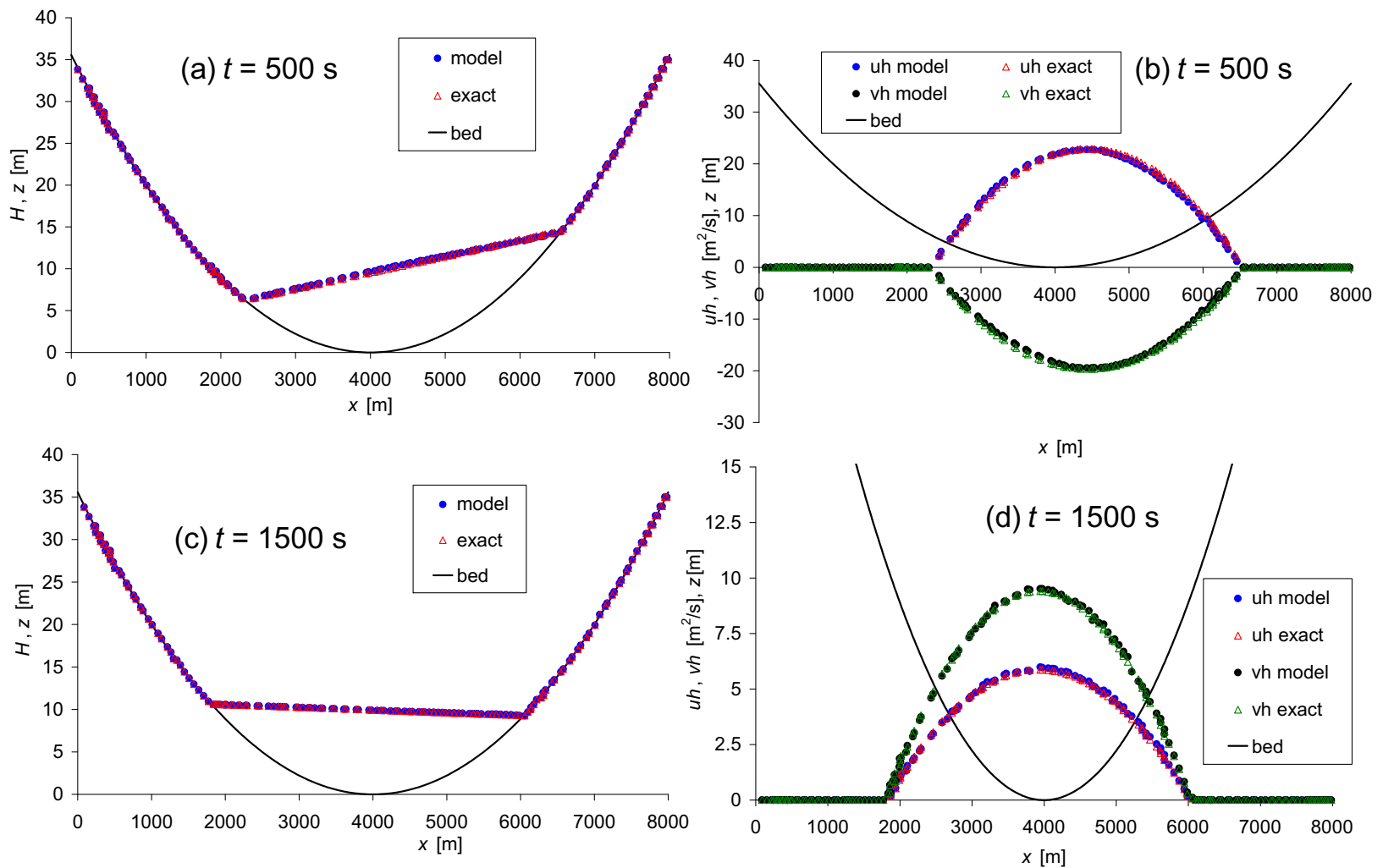

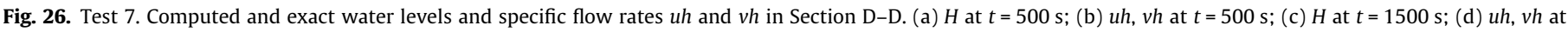
$t=1500 \mathrm{~s}$. 
Table 2

Mean CPU times per iteration (in seconds).

\begin{tabular}{llll}
\hline$N_{T}$ & Cell ordering & Prediction problem & Correction problem \\
\hline MAST & & & \\
8650 & $1.84 \mathrm{E}-06$ & $3.56 \mathrm{E}-05$ & $1.93 \mathrm{E}-06$ \\
34600 & $2.37 \mathrm{E}-06$ & $3.71 \mathrm{E}-05$ & $2.18 \mathrm{E}-06$ \\
138400 & $2.90 \mathrm{E}-06$ & $3.44 \mathrm{E}-05$ & $2.48 \mathrm{E}-06$ \\
553600 & $3.17 \mathrm{E}-06$ & $3.12 \mathrm{E}-05$ & $2.72 \mathrm{E}-06$ \\
\hline
\end{tabular}

performed as previously described and the time step size has been halved at each mesh refinement.

Table 2 shows the mean computational times (CPU, in seconds) per iteration, required for element ordering, solution of the prediction and of the correction steps. The mean CPU times have been computed by dividing the total times required by the different algorithm steps by the number of triangles $N_{T}$. Numerical runs have been performed using a single processor Intel $Q 6600$, $2.40 \mathrm{GHz}$. A brief comparison with the computational times of the previous algorithm [7] is also given, where the simulations of the scheme [7] have been performed on the same processor.

The growth rate $\beta$ of the CPU time is measured as the power exponent of the relationship:

$\overline{C P U}=\left(N_{T}\right)^{\beta} \Rightarrow \log (\overline{C P U})=\log \left(N_{T}\right) \cdot \beta$,

where $\overline{C P U}$ is the mean CPU time per iteration.

The computation of the prediction step is the most demanding one, but it is almost independent from the mesh elements number, since this represents the "explicit" component of the method. The proposed procedure requires a computational cost for the solution of the prediction step approximately $80 \%$ of the one required by the previous MAST algorithm [7]. This is essentially due to the lack, in the proposed procedure, of the extra correction step solution as in the algorithm in [7]. The small decrement of the average CPU time for the prediction step can be related to the increasing CFL numbers obtained by partitioning and to the best aptitude of the algorithm to work with CFL numbers greater than 1 [7]. Rates $\beta$ of the prediction step are negative and their absolute values are much less than 1, as shown in Fig. 27. The mean CPU time for the correction step solution is one magnitude order less than the time required for the prediction step, but increases with the element number. In fact this step, representing the "non explicit" component of the algorithm, require the solution of large linear systems of the order of the elements number. The growth is much less than linear, with a rate $\beta$ approximately equal to 0.0841 (see Fig. 27). Element ordering requires, in the new scheme, CPU times very similar to the ones required for the solution of the correction step and almost $22 \%$ the ones of the previous scheme, where an extra linear system had to be solved [7]. Element ordering CPU time

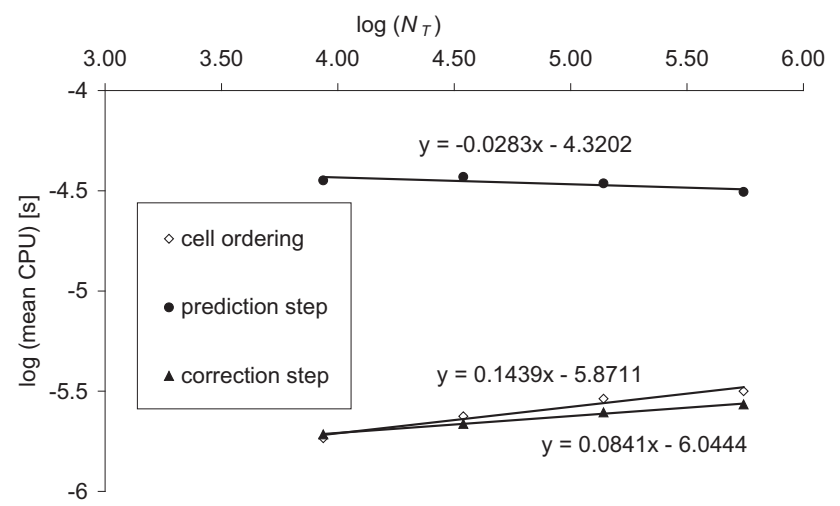

Fig. 27. Mean CPU times for the different model steps and relative growth exponents $\beta$ (see Eq. (71)). increases with element number, but its growth is much less than linear, with a rate $\beta$ equal to 0.1439 .

\section{Conclusions}

A novel procedure has been proposed for the numerical solution of the 2D fully dynamic form of the shallow water equations, starting from the numerical structure of the previous MAST algorithm [7]. Comparison with experimental data and analytical tests (some of them not documented in the present paper) show a significant improvement with respect to the previous algorithm, but we believe that the most important advance of the research is the development of the anisotropic potential and the $O$ property concepts, where the $O$ property is the counterpart of the anisotropic potential for a discretized flow field.

Starting from these definitions, we have shown that it is possible to develop a simple algorithm where the techniques available for the solution of irrotational flow problems can be easily adapted to the solution of the most general Reynolds equations.

A final very important issue for future investigations is the parallelization of the convective step in the MAST algorithm. This step can be solved simultaneously for all the elements with the same order number, and this should make the minimum computational effort of each processor basically proportional to the number of elements crossed by each stream line.

\section{Appendix A. Loops cannot occur in discontinuous velocity fields}

Let $\mathbf{u}$ be smooth in time and space along the streamline, with the exception of the point with co-ordinates $\mathbf{x}_{d}(t)$. Let $a$ and $b$ be two points on the same streamline with co-ordinate vectors $\mathbf{x}_{a}$ and $\mathbf{x}_{b}$ such that $s_{a}<s_{d}<s_{b}$. The difference between potential $P$ in the two points is given by:

$P\left(\mathbf{x}_{b}\right)-P\left(\mathbf{x}_{a}\right)=\int_{a}^{s_{d}} \frac{\nabla P \cdot \mathbf{u}}{|\mathbf{u}|} d s+\int_{s_{d}}^{b} \frac{\nabla P \cdot \mathbf{u}}{|\mathbf{u}|} d s$.

Equation (A.1) coupled with Eq. (2) provides:

$P\left(\mathbf{x}_{b}\right)-P\left(\mathbf{x}_{a}\right)=-\left(\int_{a}^{s_{d}} \frac{\left(\mathbf{K}^{-1} \mathbf{u}\right) \cdot \mathbf{u}}{|\mathbf{u}|} d s+\int_{s_{d}}^{b} \frac{\left(\mathbf{K}^{-1} \mathbf{u}\right) \cdot \mathbf{u}}{|\mathbf{u}|} d s\right)$,

where, as for Eq. (11), the argument of the two integrals on the r.h.s. is always positive because $\mathbf{K}^{-1}$ is positive definite. This implies that the difference $P\left(\mathbf{x}_{b}\right)-P\left(\mathbf{x}_{a}\right)$ is always negative, unless velocity $\mathbf{u}$ is zero along all the streamline. Moreover, due to the assumption of continuity and smoothness of velocity $\mathbf{u}$, far from $\mathbf{x}_{d}(t)$, Eq. (A.2) proves that $P$ is a continuous function along the streamline, with a jump in its first order derivative at $s_{d}$. This also implies that a closed streamline cannot occur in the flow field.

\section{Appendix B. The fractional time step methodology}

Eqs. (15)-(17) can be solved by means of a fractional time step approach, by splitting the original problem in a prediction and a correction problem. Assume a general system of balance laws:

$\frac{\partial \mathbf{U}}{\partial t}+\nabla \cdot \mathbf{F}(\mathbf{U})=\mathbf{B}(\mathbf{U})+\nabla \cdot \mathbf{E}(\mathbf{U})$,

where $\mathbf{U}$ is the vector of the unknown variables, $\mathbf{F}(\mathbf{U})$ is the inviscid flux vector, $\mathbf{E}(\mathbf{U})$ is the viscous flux vector and $\mathbf{B}(\mathbf{U})$ is a source term. Applying a fractional time step procedure, we set:

$$
\begin{aligned}
& \mathbf{F}(\mathbf{U})=\mathbf{F}^{p}(\mathbf{U})+\left(\mathbf{F}(\mathbf{U})-\mathbf{F}^{p}(\mathbf{U})\right), \\
& \mathbf{E}(\mathbf{U})=\mathbf{E}^{p}(\mathbf{U})+\left(\mathbf{E}(\mathbf{U})-\mathbf{E}^{p}(\mathbf{U})\right), \\
& \mathbf{B}(\mathbf{U})=\mathbf{B}^{p}(\mathbf{U})+\left(\mathbf{B}(\mathbf{U})-\mathbf{B}^{p}(\mathbf{U})\right),
\end{aligned}
$$


where $\mathbf{F}^{p}(\mathbf{U}), \mathbf{E}^{p}(\mathbf{U})$ and $\mathbf{B}^{p}(\mathbf{U})$ are respectively suitable numerical inviscid flux, viscous flux and source terms, further defined. After integration in space, system (B.1) can be split in the two following ones:

$$
\begin{aligned}
& \mathbf{U}^{k+1 / 2}-\mathbf{U}^{k}+\nabla \cdot \int_{0}^{\Delta t} \mathbf{F}^{p} d t=\int_{0}^{\Delta t} \mathbf{B}^{p} d t+\nabla \cdot \int_{0}^{\Delta t} \mathbf{E}^{p} d t, \\
& \mathbf{U}^{k+1}-\mathbf{U}^{k+1 / 2}+\nabla \cdot \int_{0}^{\Delta t} \mathbf{F} d t-\nabla \cdot \overline{\mathbf{F}}^{p} \Delta t \\
& =\int_{0}^{\Delta t} \mathbf{B} d t-\overline{\mathbf{B}}^{p} \Delta t+\nabla \cdot \int_{0}^{\Delta t} \mathbf{E} d t-\nabla \cdot \overline{\mathbf{E}}^{\mathbf{p}} \Delta \mathbf{t},
\end{aligned}
$$

where $\overline{\mathbf{F}}^{p}, \overline{\mathbf{E}}^{p}$ and $\overline{\mathbf{B}}^{p}$ are the mean in time values of $\mathbf{F}^{p}(\mathbf{U}), \mathbf{E}^{p}(\mathbf{U})$ and $\mathbf{B}^{p}(\mathbf{U})$ computed along the prediction step, $\mathbf{U}^{k+1 / 2}$ and $\mathbf{U}^{k+1}$ are the unknown variables computed respectively at the end of the prediction and the correction phase. $\overline{\mathbf{F}}^{p}, \overline{\mathbf{E}}^{p}$ and $\overline{\mathbf{B}}^{p}$ are estimated "a posteriori" after the solution of the prediction problem. We call systems (B.3,a) and (B.3,b) prediction and correction systems respectively. Observe that summing systems (B.3,a) and (B.3,b), the integral of the original system (B.1) is formally obtained. The difference between $\mathbf{U}^{k+1}$ and $\mathbf{U}^{k+1 / 2}$ in Eq. (B.3,b) is close to zero as far as the difference between the predicted and mean in time values of the fluxes and source terms is either small or time-independent. The advantage of using formulations (B.3) instead of (B.1) is that, with a suitable choice of the prediction terms $\mathbf{F}^{p}(\mathbf{U}), \mathbf{E}^{p}(\mathbf{U})$ and $\mathbf{B}^{p}(\mathbf{U})$, each of the two systems (B.3,a) and (B.3,b) can be much easier to solve than the original system (B.1). In the present case we have:

$$
\begin{aligned}
& \mathbf{U}=\left(\begin{array}{lll}
h & u h & v h
\end{array}\right)^{T} \quad \mathbf{F}=\left(\begin{array}{ll}
\mathbf{F}_{\mathbf{1}} & \mathbf{F}_{\mathbf{2}}
\end{array}\right) \quad \mathbf{E}=\left(\begin{array}{ll}
\mathbf{E}_{\mathbf{1}} & \mathbf{E}_{\mathbf{2}}
\end{array}\right), \\
& \mathbf{F}_{\mathbf{1}}=\left(\begin{array}{c}
u h \\
u^{2} h+\frac{1}{2} g h^{2} \\
u v h
\end{array}\right) \quad \mathbf{F}_{\mathbf{2}}=\left(\begin{array}{c}
v h \\
u v h \\
v^{2} h+\frac{1}{2} g h^{2}
\end{array}\right) \quad \mathbf{E}_{\mathbf{1}}=\left(\begin{array}{c}
0 \\
v h \frac{\partial u}{\partial x} \\
v h \frac{\partial v}{\partial x}
\end{array}\right) \quad \mathbf{E}_{\mathbf{2}}=\left(\begin{array}{c}
0 \\
v h \frac{\partial u}{\partial y} \\
v h \frac{\partial v}{\partial y}
\end{array}\right),
\end{aligned}
$$

$$
\mathbf{B}=\left(\begin{array}{cc}
0 & -g h\left(\frac{\partial z}{\partial x}+\frac{n^{2} u \sqrt{(u h)^{2}+(v h)^{2}}}{h^{7 / 3}}\right) \\
& -g h\left(\frac{\partial z}{\partial y}+\frac{n^{2} v \sqrt{(u h)^{2}+(v h)^{2}}}{h^{7 / 3}}\right)
\end{array}\right)^{T}
$$

$$
\mathbf{F}_{1}^{p}=\left(\begin{array}{c}
u h \\
u^{2} h \\
u v h
\end{array}\right) \quad \mathbf{F}_{2}^{p}=\left(\begin{array}{c}
v h \\
u v h \\
v^{2} h
\end{array}\right) \quad \mathbf{E}_{1}^{p}=\left(\begin{array}{c}
0 \\
v h^{k} \frac{\partial u^{k}}{\partial x} \\
v h^{k} \frac{\partial v^{k}}{\partial x}
\end{array}\right) \quad \mathbf{E}_{2}^{p}=\left(\begin{array}{c}
0 \\
v h^{k} \frac{\partial u^{k}}{\partial y} \\
v h^{k} \frac{\partial v^{k}}{\partial y}
\end{array}\right)
$$

$$
\mathbf{B}^{p}=\left(\begin{array}{cc}
0 & -g h\left(\frac{\partial H^{k}}{\partial x}+\frac{n^{2} u \sqrt{(u h)^{2}+(v h)^{2}}}{h^{7 / 3}}\right) \\
& -g h\left(\frac{\partial H^{k}}{\partial y}+\frac{n^{2} v \sqrt{(u h)^{2}+(v h)^{2}}}{h^{7 / 3}}\right)
\end{array}\right)^{T}
$$

where index $k$ marks the beginning of the time step (time level $t^{k}$ ) and $(.)^{T}$ is the transposed of vector (.). Observe that the total head gradient in the $\mathbf{B}^{p}$ vector, as well as the gradients of velocity components in the $\mathbf{E}^{p}$ vector are computed at time level $t^{k}$ and are kept constant along the time step.

Prediction problem is solved in its integral form, while the correction problem is solved in its differential linearized form.

\section{Appendix C. Computational properties of the mesh}

Let $i$, ip and im be the nodes of triangle $T_{e}$, where ip and $i m$ are the nodes respectively following and preceding node $i$ in counterclockwise direction, as specified in Section 4. Let $T_{e p}$ be the triangle sharing side $\mathbf{r}_{i, i p}$ with $T_{e}$. We compute $c_{i, i p}^{T_{e}}$, the distance between the $T_{e}$ circumcentre $c_{T_{e}}$ and the midpoint of $\mathbf{r}_{i, i p}$, as (see Fig. 3):

$$
\begin{aligned}
& c_{j, j p}^{T_{q}}=\frac{\left(x_{j}-x_{j p}\right)\left(y_{c_{q}}-y_{j, j p}\right)-\left(y_{j}-y_{j p}\right)\left(x_{c_{q}}-x_{j, j p}\right)}{\sqrt{\left(x_{j}-x_{j p}\right)^{2}+\left(y_{j}-y_{j p}\right)^{2}}} \delta_{q}, \\
& q=\left\{\begin{array}{l}
e \\
e p
\end{array} \Rightarrow j=\left\{\begin{array}{l}
i \\
i p
\end{array}, j p=\left\{\begin{array}{l}
i p \\
i
\end{array}\right.\right.\right.
\end{aligned}
$$

where $\mathbf{x}_{j, j p}$ is the co-ordinate vector of midpoint of side $\mathbf{r}_{j, j p}, \mathbf{x}_{c_{q}}$ is the co-ordinate vector of circumcentre of triangle $T_{q}, \delta_{q}=-1$ or 1 if direction of vector $\mathbf{r}_{j, j p}$ is respectively counterclockwise or not in triangular element $T_{q}$, with $q, j$ and $j p$ defined in Eq. (C.1).

We say a mesh to satisfy the generalised Delaunay (GD) property when all the sides satisfy the constraints:

$c_{i, i p}^{T_{e}}+c_{i p, i}^{T_{e p}} \geqslant 0$ or $c_{i, i p}^{T_{e}} \geqslant 0$,

for each interior or boundary edge, respectively, with nodes $i$ and ip (see $[8,9]$ and cited references). Fig. 4(a) shows an internal side satisfying the GD property. Most of the today available mesh-generators satisfy the GD property, even if some exceptions may occur around internal boundaries, or when the mesh density is forced to change in given sub-domains. If the GD property is not satisfied (as in the example shown in Fig. 4(b)), it is still possible to obtain a new mesh that satisfies the GD property without changing the location of the original nodes, for example by swapping edges, applying the procedure presented in [9].

We will show in the following sections that the GD mesh condition, along with a special formulation of matrix coefficients for heterogeneous medium, implies the so called $M$-property [48] for the resulting matrix of the linear system of the diffusive problem. An $M$-matrix is an irreducible matrix, with positive diagonal coefficients and non-positive off-diagonal coefficients, strictly diagonally dominant, or weakly diagonally dominant with strict inequality for at least one row. The $M$-property guarantees interelement fluxes with a sign that is always consistent with the sign of the corresponding water level difference. An important consequence is the monotonicity of the steady-state solution, when source terms are missing, as well as the lack of spatial oscillations [48].

\section{References}

[1] Abbott MB, Minns AW. Computational hydraulics. 2nd ed. Ashgate Edt.; 1998.

[2] Aizinger V, Dawson CN. A discontinuous Galerkin method for two-dimensional flow and transport in shallow water. Adv Water Res 2002;25:67-84. http:/l dx.doi.org/10.1016/S0309-1708(01)00019-7.

[3] Alcrudo F, Garcia-Navarro P. A high-resolution Godunov-type scheme in finite volumes for the 2D shallow water equations. Int J Numer Methods Fluids 1993;16:489-505. http://dx.doi.org/10.1002/fld.1650160604.

[4] Anastasiou K, Chan CT. Solution of the 2D shallow water equations using the finite volume method on unstructured triangular meshes. Int J Numer Methods Fluids 1997;24(11):1225-45. $\quad$ http://dx.doi.org/10.1002/(SICI)10970363(19970615)24:11<1225::AID-FLD540>3.0.CO;2-D.

[5] Aricò C, Tucciarelli T. MAST solution of advection problems in irrotational flow fields. Adv Water Res 2007;30(3):665-85. http://dx.doi.org/10.1016/ j.advwatres.2006.03.007.

[6] Aricò C, Tucciarelli T. A Marching in space and time (MAST) solver of the shallow water equations. Part I: The 1D model. Adv Water Res 2007;30(5):1236-52. http://dx.doi.org/10.1016/i.advwatres.2006.11.0.

[7] Aricò C, Nasello C, Tucciarelli T. A marching in space and time (MAST) solver of the shallow water equations. Part II: The 2D model. Adv Water Res 2007;30(5):1253-71. http://dx.doi.org/10.1016/j.advwatres.2006.11.0.

[8] Aricò C, Sinagra M, Begnudelli L, Tucciarelli T. MAST-2D diffusive model for flood prediction on domains with triangular Delaunay unstructured meshes. Adv Water Res 2011;34(11):1427-49. http://dx.doi.org/10.1016/ j.advwatres.2011.08.002.

[9] Aricò C, Sinagra M, Tucciarelli T. Monotonic solution of flow and transport problems in heterogeneous media using Delaunay unstructured triangular meshes. Adv Water Res 2013;52:132-50. http://dx.doi.org/10.1016/ j.advwatres.2012.09.006. 
[10] Bascià A, Tucciarelli T. An explicit unconditionally stable numerical solution of the advection problem in irrotational flow fields. Water Resour Res 2004;40(6):W06501. http://dx.doi.org/10.1029/2003WR002646.

[11] Bermúdez A, Vázquez ME. Upwind methods for hyperbolic conservation laws with source terms. Comput Fluids 1994;23(8):1049-71. http://dx.doi.org/ 10.1016/0045-7930(94)90004-3.

[12] Brufau P. Simulaciòn bidimensional de flujos hidrodinàmicos transitorios en geometrìas irregulares. Ph.D. thesis, Universidad de Zaragoza, 2000

[13] Brufau P, Vazquez-Cendon ME, Garcia-Navarro P. A numerical model for the flooding and dryingof irregular domains. Int $\mathrm{J}$ Numer Methods Fluids 2002;39:247-75. http://dx.doi.org/10.1002/fld.285.

[14] Canestrelli A, Dumbser M, Siviglia A, Toro EF. Well-balanced high-order centered schemes on unstructured meshes for shallow water equations with fixed and mobile bed. Adv Water Res 2010;33:291-303. http://dx.doi.org/ 10.1016/i.advwatres.2009.12.006.

[15] Chang SC. The method of space-time conservation element and solution element - a new approach for solving the Navier-Stokes and Euler equations. J Comput Phys 1995;119:295-324. http://dx.doi.org/10.1006/ icph.1995.1137.

[16] Chippada S, Dawson CN, Martinez M, Wheeler M. A Godunov-type finite volume method for the system of shallow water equations. Comput Methods Appl Mech Eng 1998;151:105-29. http://dx.doi.org/10.1016/S00457825(97)00108-4.

[17] Dawson CN, Proft J. Discontinuous and coupled continuous/discontinuous Galerkin methods for the shallow water equations. Comput Methods Appl Mech Eng 2002;191:4721-46. http://dx.doi.org/10.1016/S00457825(02)00402-4.

[18] Fraccarollo L, Toro EF. Experimental and numerical assessment of the shallow water model for two-dimensional dam-break type problems. J Hydraul Res 1995;33(6):843-64. http://dx.doi.org/10.1080/00221689509498555.

[19] Gottardi G, Venutelli M. Central scheme for two-dimensional dam-break flow simulation. Adv Water Res 2004;27:259-68. http://dx.doi.org/10.1016/ j.advwatres.2003.12.006.

[20] Gourgue O, Comblen R, Lambrechts J, Kärnä T, Legat V, Deleersnijder E. A fluxlimiting wetting-drying method for finite-element shallow-water models, with application to the Scheldt Estuary. Adv Water Res 2009;32:1726-39. http://dx.doi.org/10.1016/i.advwatres.2009.09.005.

[21] Goutal N, Maurel F, editors. Proceedings of the second workshop on dam-break wave simulation. HE-43/97/016/B, France; 1997.

[22] Greenberg JM, LeRoux AY. A well-balanced scheme for the numerical processing of source terms in hyperbolic equations. SIAM J Numer Anal 1996;33:1-16. http://dx.doi.org/10.1137/0733001.

[23] Hou J, Liang Q, Simons F, Hinkelmann R. A 2D well-balanced shallow flow model for unstructured grids with novel slope source term treatment. Adv Water Res 2013;52:107-31. http://dx.doi.org/10.1016/ j.advwatres.2012.08.003.

[24] Hubbard ME. Multidimensional slope limiters for MUSCL-type finite volume schemes on unstructured grids. J Comput Phys 1999;155(1):54-74. http:// dx.doi.org/10.1006/icph.1999.6329.

[25] Hubbard ME, Garcìa-Navarro P. Flux difference splitting and the balancing of source terms and flux gradients. J Comput Phys 2000;165(1):89-125. http:// dx.doi.org/10.1006/jicph.2000.6603.

[26] Ivanenko SA, Muratova GV. Adaptive grid shallow water modelling. Appl Numer Math 2000;32:447-82. http://dx.doi.org/10.1016/S01689274(99)00063-X.

[27] Kärnä T, de Brye B, Gourgue O, Lambrechts J, Comblen R, Legat V, Deleersnijder E. A fully implicit wetting-drying method for DG-FEM shallow water models, with an application to the Scheldt Estuary. Comput Methods Appl Mech Eng 2011;200(5-8):509-24. http://dx.doi.org/10.1016/i.cma.2010.07.001.

[28] Kawahara M, Hirano H, Tsubota K, Inagaki K. Selective lumping finite element method for shallow water flow. Int J Numer Methods Fluids 1982;2:89-112. http://dx.doi.org/10.1002/fld.1650020106.

[29] Kurganov A, Tadmor E. New high-resolution central schemes for non-linear conservation laws and convection-diffusion equations. J Comput Phys 2000;160:241-82. http://dx.doi.org/10.1006/icph.2000.6459.

[30] Lai C. Numerical modeling of unsteady open-channel flow. Adv Hydrosc $1986 ; 14: 161-333$.
[31] LeVeque RJ. Balancing source terms and flux gradients in high-resolution Godunov methods: the quasi steady wave propagation algorithm. J Comput Phys 1998;146:346-65. http://dx.doi.org/10.1006/icph.1998.6058.

[32] Liang Q Borthwick AGL. Adaptive quadtree simulation of shallow flows with wet-dry fronts over complex topography. Comput Fluids 2009;38:221-34. http://dx.doi.org/10.1016/i.compfluid.2008.02.008.

[33] Liang Q Marche F. Numerical resolution of well-balanced shallow water equations with complex source terms. Adv Water Res 2009;32:873-84. http:// dx.doi.org/10.1016/j.advwatres.2009.02.010.

[34] Lynch DR, Gray WR. A wave equation model for finite element tida computations. Comput Fluids 1979;7:207-28. http://dx.doi.org/10.1016 0045-7930(79)90037-9.

[35] Mohamadian A, Le Roux DY, Tajrishi M, Mazaheri K. A mass conservative scheme for simulating shallow flows over variable topographies using unstructured grid. Adv Water Res 2005;28:523-39. http://dx.doi.org 10.1016/i.advwatres.2004.10.006.

[36] Nag Library Manual; 2005 <http://www.nag.co.uk/numeric/fl/manual/html/ mark21.html>

[37] Ritter A. Die Fortplanzung der wasserwellen. Z Ver Dtsch Ing 1992;36(33):947-54.

[38] Rogers B, Fujihara M, Borthwick AGL. Adaptive Q-tree Godunov-type scheme for shallow water equations. Int J Numer Methods Fluids 2001;35:247-80. http: dx.doi.org/10.1002/1097-0363(20010215)35:3<247::AID-FLD89>3.0.CO:2-E.

[39] Saint-Venant B. Théorie du mouvement non permanent des eaux, avec application aux crues des rivières et à l'introduction des marées dans leurs lits. CR Séances Acad Sci 1871;73. 147-154, 237-240.

[40] Sampson J, Easton A, Singh M. Moving boundary shallow water flow in circular paraboloidal basins. In: Proceedings of the sixth engineering mathematics and applications conference, Sydney Australia; 2003. p. 223-227.

[41] Singh J, Altinakar M, Ding Y. Two-dimensional numerical modeling of dambreak flows over natural terrain using a central explicit scheme. Adv Water Res 2011;34:1366-75. http://dx.doi.org/10.1016/j.advwatres.2011.07.007.

[42] Skoula ZD, Borthwick AGL, Moutzouris CI. Godunov-type solution of the shallow water equations on adaptive unstructured triangular grids.. Int J Comput Fluid Dyn 2006;20(9):621-36. http://dx.doi.org/10.1080 10618560601088327.

[43] Soares Frazão S, Sillen X, Zech Y. Dam-break flow through sharp bends physica model and 2D Boltzmann model validation, Wallingford CADAM meeting, 2-3 March 1998. <http://www.hrwallingford.co.uk/projects/CADAM/CADAM index.html>.

[44] Szymkiewicz R. Oscillation-free solution of shallow water equations for non staggered grid. J Hydraul Eng ASCE 1993;119:1118-37. http://dx.doi.org 10.1061/(ASCE)0733-9429(1993)119:10(1118).

[45] Szymkiewicz R. Numerical Modeling in Open Channel Hydraulics. Springer; 2010.

[46] Vazquez-Cendon ME. Improved treatment of source terms in upwind schemes for shallow water equations in channels with irregular geometry. J Comput Phys 1999;148:497-526. http://dx.doi.org/10.1006/jcph.1998.6127.

[47] Wang JW, Liu RX. Combined finite volume-finite element method for shallow water equations. Comput Fluids 2005;34:1199-222. http://dx.doi.org 10.1016/i.compfluid.2004.09.008.

[48] Younes A, Ackerer P, Lehmann F. A new mass lumping scheme for the mixed hybrid finite element method. Int J Numer Methods Eng 2006;67:89-107. http://dx.doi.org/10.1002/nme.1628.

[49] Zhang Y, Zeng Z, Chen J. The improved space-time conservation element and solution element scheme for two-dimensional dam-break flow simulation. Int J Numer Methods Fluids 2012;68:605-24. http://dx.doi.org/10.1002/fld.2525.

[50] Zhou JG, Causon DM, Mingham CG, Ingram DM. The surface gradient method for the treatment of the source terms in the shallow water equations. J Comput Phys 2001;168:1-25. http://dx.doi.org/10.1006/jcph.2000.6670.

[51] Zhou JG, Causon DM, Ingram DM, Mingham CG. Numerical solutions of the shallow water equations with discontinuous bed topography. Int J Numer Methods Fluids 2002;38:769-88. http://dx.doi.org/10.1002/fld.243.

[52] Zienkiewicz O, Ortiz P. A split-characteristic based finite element model for the shallow water equations. Int J Numer Methods Fluids 1995;20:1061-80. http://dx.doi.org/10.1002/fld.1650200823. 